University of Wollongong

Research Online

Australian Institute for Innovative Materials -

Papers

Australian Institute for Innovative Materials

$1-1-2012$

High performance ferroelectric relaxor-PbTiO3 single crystals: Status and perspective

Shujun Zhang

Pennsylvania State University, shujun@uow.edu.au

Fei Li

Xi'an Jiaotong University, lifei1216@gmail.com

Follow this and additional works at: https://ro.uow.edu.au/aiimpapers

Part of the Engineering Commons, and the Physical Sciences and Mathematics Commons

Research Online is the open access institutional repository for the University of Wollongong. For further information contact the UOW Library: research-pubs@uow.edu.au 


\title{
High performance ferroelectric relaxor-PbTiO3 single crystals: Status and perspective
}

\author{
Abstract \\ Ferroelectrics are essential components in a wide range of applications, including ultrasonic transducers, \\ sensors, and actuators. In the single crystal form, relaxor-PbTiO3 (PT) piezoelectric materials have been \\ extensively studied due to their ultrahigh piezoelectric and electromechanical properties. In this article, a \\ perspective and future development of relaxor-PT crystals are given. Initially, various techniques for the \\ growth of relaxor-PT crystals are reviewed, with crystals up to $100 \mathrm{~mm}$ in diameter and $200 \mathrm{~mm}$ in length \\ being readily achievable using the Bridgman technique. Second, the characterizations of dielectric and \\ electromechanical properties are surveyed. Boundary conditions, including temperature, electric field, and \\ stress, are discussed in relation to device limitations. Third, the physical origins of the high piezoelectric \\ properties and unique loss characteristics in relaxor-PT crystals are discussed with respect to their \\ crystal structure, phase, engineered domain configuration, macrosymmetry, and domain size. Finally, \\ relaxor-PT single crystals are reviewed with respect to specific applications and contrasted to \\ conventional piezoelectric ceramics.

\section{Disciplines} \\ Engineering | Physical Sciences and Mathematics

\section{Publication Details} \\ Zhang, S. \& Li, F. (2012). High performance ferroelectric relaxor-PbTiO3 single crystals: Status and \\ perspective. Journal Of Applied Physics, 111 (3), 031301-1-031301-50.
}




\section{High performance ferroelectric relaxor- $\mathrm{PbTiO}_{3}$ single crystals: Status and perspective}

Shujun Zhang, and Fei Li

Citation: Journal of Applied Physics 111, 031301 (2012); doi: 10.1063/1.3679521

View online: https://doi.org/10.1063/1.3679521

View Table of Contents: http://aip.scitation.org/toc/jap/111/3

Published by the American Institute of Physics

\section{Articles you may be interested in}

Ultrahigh strain and piezoelectric behavior in relaxor based ferroelectric single crystals Journal of Applied Physics 82, 1804 (1997); 10.1063/1.365983

Electrostrictive effect in ferroelectrics: An alternative approach to improve piezoelectricity Applied Physics Reviews 1, 011103 (2014); 10.1063/1.4861260

Elastic, piezoelectric, and dielectric properties of multidomain $0.67 \mathrm{~Pb}\left(\mathrm{Mg}_{1 / 3} \mathrm{Nb}_{2 / 3}\right) \mathrm{O}_{3}-0.33 \mathrm{PbTiO}_{3}$ single crystals

Journal of Applied Physics 90, 3471 (2001); 10.1063/1.1390494

A morphotropic phase boundary system based on polarization rotation and polarization extension Applied Physics Letters 97, 062906 (2010); 10.1063/1.3479479

Composition and phase dependence of the intrinsic and extrinsic piezoelectric activity of domain engineered $(1-x) \mathrm{Pb}\left(\mathrm{Mg}_{1 / 3} \mathrm{Nb}_{2 / 3}\right) \mathrm{O}_{3}-x \mathrm{PbTiO}_{3}$ crystals

Journal of Applied Physics 108, 034106 (2010); 10.1063/1.3466978

Ferroelectric thin films: Review of materials, properties, and applications

Journal of Applied Physics 100, 051606 (2006); 10.1063/1.2336999

\section{PHYSICS TODAY}

MANAGER'S GUIDE

WHITEPAPERS
Accelerate R\&D with Multiphysics Simulation

\section{READ NOW}

PRESENTED BY

○СOMSOL 


\title{
High performance ferroelectric relaxor- $\mathrm{PbTiO}_{3}$ single crystals: Status and perspective
}

\author{
Shujun Zhang ${ }^{1, a)}$ and Fei $\mathrm{Li}^{1,2}$ \\ ${ }^{1}$ Materials Research Institute, Pennsylvania State University, University Park, Pennsylvania 16802, USA \\ ${ }^{2}$ Electronic Materials Research Laboratory and International Center for Dielectric Research, \\ Xi' an Jiaotong University, Xi' an 710049, China
}

(Received 7 October 2011; accepted 4 January 2012; published online 7 February 2012)

\begin{abstract}
Ferroelectrics are essential components in a wide range of applications, including ultrasonic transducers, sensors, and actuators. In the single crystal form, relaxor- $\mathrm{PbTiO}_{3}$ (PT) piezoelectric materials have been extensively studied due to their ultrahigh piezoelectric and electromechanical properties. In this article, a perspective and future development of relaxor-PT crystals are given. Initially, various techniques for the growth of relaxor-PT crystals are reviewed, with crystals up to $100 \mathrm{~mm}$ in diameter and $200 \mathrm{~mm}$ in length being readily achievable using the Bridgman technique. Second, the characterizations of dielectric and electromechanical properties are surveyed. Boundary conditions, including temperature, electric field, and stress, are discussed in relation to device limitations. Third, the physical origins of the high piezoelectric properties and unique loss characteristics in relaxor-PT crystals are discussed with respect to their crystal structure, phase, engineered domain configuration, macrosymmetry, and domain size. Finally, relaxor-PT single crystals are reviewed with respect to specific applications and contrasted to conventional piezoelectric ceramics.
\end{abstract}

(C) 2012 American Institute of Physics. [doi:10.1063/1.3679521]

\section{TABLE OF CONTENTS}

\section{INTRODUCTION AND BACKGROUND .......}

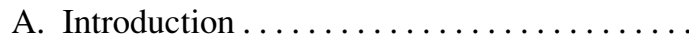

B. Background on piezoelectricity and ferroelectricity ............... 2

1. Piezoelectricity and related parameters ..

a. Dielectric permittivity ............

b. Piezoelectric coefficients..........

c. Frequency constant and elastic

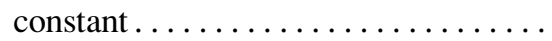

d. Acoustic impedance .............

e. Electromechanical coupling ........

f. Mechanical quality factor .........

2. Ferroelectricity and related phenomena..

a. Ferroelectric domains and domain walls ................... 4

b. Ferroelectric hysteresis loop........

c. Polymorphotropic phase transitions and morphotropic phase boundary

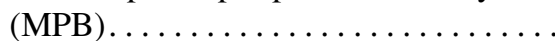

d. Domain engineering.............

e. Aging behavior and piezoelectric nonlinearity ..............

C. Background on pervoskite ferroelectric materials ................. 6

\footnotetext{
${ }^{\text {a) }}$ Author to whom correspondence should be addressed. Electronic addressess: soz1@psu.edu and shujunzhang@gmail.com.
}

2
1. History of pervoskite ferroelectric ceramics ......................... 6

2. Relaxor-PT single crystals ......... 7
II. SINGLE CRYSTAL GROWTH: ISSUES AND

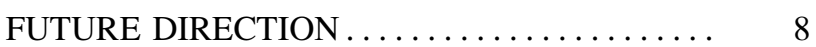

A. High temperature solution growth ...... 8

1. Conventional flux method .......... 8

2. Flux Bridgman ................ 9

B. Modified Bridgman ............... 10

C. Solid state conversion ............. 12

III. STRUCTURE AND PROPERTY

A. Crystal phase determination ........... 12

1. Microscopic characterization ......... 12

2. Macroscopic characterization........ 13

a. [111] poled relaxor-PT crystals ...... 13

b. [011] poled relaxor-PT crystals ..... 13

c. [001] poled relaxor-PT crystals ..... 13

B. Dielectric and piezoelectric measurements . . 13

C. Loss determination ............... 13

1. Hysteresis loop measurements. ........ 14

2. Impedance spectrum measurements .... 14

D. Determination of full matrix material constants......................... 14

E. Piezoelectric properties as function of orientation and composition .......... 15

1. Orientation dependent properties....... 15

2. Composition dependent properties ..... 18

F. Pyroelectric and electro-optic properties .... 19
CHARACTERIZATIONS . . . . . . . . . . . . 12

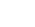
8

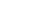
(10

2

12

12

3

(3)

3

4

4

\section{4} 5 15 19

9


IV. ORIGIN OF PIEZOELECTRIC RESPONSE AND LOSSES.

A. Piezoelectric properties ............. 20

1. Electric field induced phase transitions .. 20

2. Polarization rotation mechanism...... 20 "Polarization rotation" vs "domain wall motion"......................

3. High shear piezoelectric response and MPB.........................

4. The role of a monoclinic phase........

5. The role of relaxor end member........

6. Critical factors for high piezoelectricity .

B. Loss in relaxor-PT crystals ..............

1. Internal bias and domain wall motion ...

2. Polarization rotation $\ldots \ldots \ldots \ldots \ldots \ldots$

3. Polarization rotation angle...........

4. Morphotropic phase boundary .........

5. Losses under high $a c$ drive field........

V. PROPERTIES UNDER EXTERNAL BOUNDARY CONDITIONS.

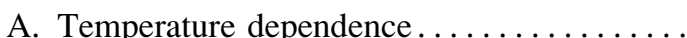

B. Uniaxial stress and $d c$ bias field effects ....

1. Stress/electric field induced phase transitions ......................

2. Piezoelectric properties as a function of $d c$ bias and uniaxial stress .............

a. dc bias field ....................

b. Uniaxial stress..................

c. Temperature usage range under $\mathrm{dc}$ bias .........................

C. Hydrostatic pressure .................

D. Relaxor-PT crystals under high drive field. .

1. Dielectric and piezoelectric properties under high drive field ...............

2. Field stability of the shear properties....

3. Fatigue behavior $\ldots \ldots \ldots \ldots \ldots \ldots$.

VI. APPLICATIONS $\ldots \ldots \ldots \ldots \ldots \ldots \ldots \ldots$

A. Ultrasound transducers...............

1. Medical ultrasonic transducers ........

2. Underwater acoustic transducers........

B. Sensors ..........................

1. Hydrophones. ...................

2. Accelerometers..................

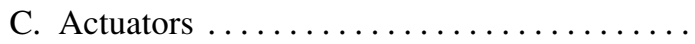

1. Stack/in-plane actuators ............

2. Flextensional actuators .............

3. Ultrasonic motors (Resonant actuators) . .

VII. SUMMARY AND FUTURE PERSPECTIVE. .

A. Summary $\ldots \ldots \ldots \ldots \ldots \ldots \ldots \ldots \ldots$

B. Future perspectives ..............

\section{INTRODUCTION AND BACKGROUND}

\section{A. Introduction}

Piezoelectric materials find application in a wide range of sensors, actuators, and ultrasonic transducers that are important in various industrial and scientific areas. Innovations in electromechanical devices continue to be the driving force for the development of new piezoelectric materials. Applied piezoelectric materials include bulk ceramics, ${ }^{1,2}$ single crystals, ${ }^{3-5}$ thin films, ${ }^{6-8}$ textured ceramics, ${ }^{9}$ polymers, ${ }^{10}$ and composites, ${ }^{11-14}$ in which, relaxor based ferroelectric single crystals, such as $\mathrm{Pb}\left(\mathrm{Mg}_{1 / 3} \mathrm{Nb}_{2 / 3}\right) \mathrm{O}_{3}-\mathrm{PbTiO}_{3}$ (PMN-PT) and $\mathrm{Pb}\left(\mathrm{Zn}_{1 / 3} \mathrm{Nb}_{2 / 3}\right) \mathrm{O}_{3}-\mathrm{PbTiO}_{3}$ ( $\left.\mathrm{PZN}-\mathrm{PT}\right)$, have attracted extensive attentions over the last $15 \mathrm{yr}$, due to their ultra-high piezoelectric properties. The longitudinal piezoelectric strain coefficient $d_{33}$ and electromechanical coupling factor $k_{33}$, being on the order of $>1500 \mathrm{pC} / \mathrm{N}$ and $\sim 0.9$, far out-perform state-of-art polycrystalline ceramics $\mathrm{Pb}(\mathrm{Zr}, \mathrm{Ti}) \mathrm{O}_{3}$ (PZT), showing great promise for various electromechanical applications. $^{3-5}$

In this review, a concise background on piezoelectricity/ ferroelectricity and their related properties are given in Sec. I. Various crystal growth methods, including high temperature solution (flux), Bridgman, and solid state crystal growth (SSCG), are surveyed and discussed in Sec. II. In Sec. III, the characterization of single crystal relaxor-PTs is given, while the origins of the ultrahigh piezoelectric properties are discussed and presented in Sec. IV. Piezoelectric materials used for electromechanical devices are generally subjected to various external conditions, including temperature, $d c$ bias field, $a c$ drive field, and prestress. The role of external boundary conditions on the properties of relaxer-PT crystals will be discussed in detail in Sec. V. The relationship of material property and device performance, contrasted with state of the art PZT ceramics and new relaxor-PT single crystals will be reviewed in Sec. VI. Finally, a brief summary and future trend are presented in Sec. VII.

\section{B. Background on piezoelectricity and ferroelectricity}

\section{Piezoelectricity and related parameters}

Piezoelectricity is the ability of certain crystalline materials, which possess non-centre-symmetries, to develop an electric polarization proportional to an applied mechanical stress (direct piezoelectric effect) or develop a mechanical strain (deformation) proportional to an applied electric field (converse piezoelectric effect). The direct piezoelectric effect was first discovered in quartz by Curie and Curie in $1880,{ }^{15}$ soon after that, the converse piezoelectric effect was predicted by Lippmann, ${ }^{16}$ based on thermodynamic considerations, and confirmed experimentally by Curie brothers in 1881.

In regard to various electromechanical applications, the following piezoelectric and related properties will be introduced, including dielectric permittivity $\varepsilon_{\mathrm{r}}$, piezoelectric coefficient $d$, frequency constant $N$ (phase velocity $v$ and elastic constant $s / c$ ), electromechanical coupling factor $k$, and mechanical quality factor $Q .{ }^{17-25}$

a. Dielectric permittivity. The electric displacement in a dielectric material is related to the applied electric field through the relation

$$
D_{i}=\varepsilon_{i j} \cdot E_{j}
$$


where $\varepsilon_{\mathrm{ij}}$ is the dielectric permittivity of the material. The relative dielectric permittivity $\varepsilon_{\mathrm{r}(\mathrm{ij})}$ (commonly refers to as the dielectric constant) can be calculated according to

$$
\varepsilon_{r(i j)}=\varepsilon_{i j} / \varepsilon_{0},
$$

where $\varepsilon_{0}$ is the permittivity of free space. In simple inorganic solids, the dielectric permittivity is mainly associated with the electronic and ionic polarizabilities, while in ferroelectric ceramics, the domain walls and polarization rotation make important contributions to the dielectric properties. ${ }^{17,18}$ The dielectric permittivity is complex number with real $\left(\varepsilon_{r}^{\prime}\right)$ and imaginary $\left(\varepsilon_{r}{ }^{\prime \prime}\right)$ components, following the equation:

$$
\varepsilon_{r}^{\prime \prime}=\varepsilon_{r}^{\prime} \cdot \tan \delta,
$$

where $\tan \delta$ is the dielectric loss.

The capacitance of the piezoelectric element can be determined by

$$
C=\varepsilon_{r} \varepsilon_{0} \cdot A / t
$$

where $A$ and $t$ are the area and thickness of the element. Determination of the dielectric permittivity is generally important for the device performance, since the capacitance is closely related to electrical impedance $Z$, following:

$$
Z=\frac{1}{j \omega C} .
$$

To allow the $50 \Omega$ electrical impedance matching, for example, single element transducer utilizes low permittivity materials, while high frequency medical array element requires high permittivity materials. ${ }^{17}$

For most practical applications, the free dielectric permittivity $\varepsilon^{T}$ (dielectric displacement in a piezoelectric element at constant stress) and clamped dielectric permittivity $\varepsilon_{\varepsilon}^{\mathrm{S}}$ (dielectric displacement at constant strain) are sufficient in theoretical and device analysis. The relationship between the two dielectric permittivities can be expressed as

$$
\varepsilon^{S}=\varepsilon^{T}-d^{2} / s^{E},
$$

where $d$ and $s$ are piezoelectric and elastic compliance constant, respectively.

b. Piezoelectric coefficients. Piezoelectricity refers to the linear coupling between mechanical stress and electric polarization and/or between mechanical strain and applied electric field. ${ }^{17,18}$ Based on the selection of independent electrical and mechanical variables, there are four sets of piezoelectric coefficients. ${ }^{19-24}$ At constant electric field (E) and mechanical stress $(\mathrm{T})$, the piezoelectric strain (or charge) coefficient $d$ is formulated as

$$
d=\left(\frac{\partial S}{\partial E}\right)_{T}=\left(\frac{\partial D}{\partial T}\right)_{E},
$$

while the piezoelectric voltage coefficient $g$ is related to the voltage response to an applied stress, where the electrical displacement (D) and $\mathrm{T}$ are selected as independent variables, following:

$$
g=\left(-\frac{\partial E}{\partial T}\right)_{D}=\left(\frac{\partial S}{\partial D}\right)_{T} .
$$

The piezoelectric strain coefficient $d$ is generally used in low frequency or non-resonant device applications, while piezoelectric stress coefficients $e$ is employed at high frequency or under mechanically clamped conditions, where $\mathrm{E}$ and strain (S) are selected as independent variables, and given by

$$
e=\left(-\frac{\partial T}{\partial E}\right)_{S}=\left(\frac{\partial D}{\partial S}\right)_{E}
$$

Finally, the piezoelectric clamped voltage (or stiffness) coefficient $h$ can be defined when the independent variables are $\mathrm{S}$ and $\mathrm{D}$, according to the equation

$$
h=\left(-\frac{\partial T}{\partial D}\right)_{S}=\left(-\frac{\partial E}{\partial S}\right)_{D} .
$$

The four sets piezoelectric coefficients are interrelated as follows:

$$
\left\{\begin{array}{l}
d_{i \lambda}=\varepsilon_{i j}^{T} g_{j \lambda}=e_{i \mu} s_{\mu \lambda}^{E} \\
e_{i \lambda}=\varepsilon_{i j}^{S} h_{j \lambda}=d_{i \mu} c_{\mu \lambda}^{E} \\
g_{i \lambda}=\beta_{i j}^{T} d_{j \lambda}=h_{i \mu} s_{\mu \lambda}^{D}, \\
h_{i \lambda}=\beta_{i j}^{S} e_{j \lambda}=g_{i \mu} c_{\mu \lambda}^{D}
\end{array},\right.
$$

where $\beta$ is the dielectric impermeability, and s/c are the elastic compliance and stiffness constants, respectively. The superscripts $E, T, S$, and $D$ indicate different boundary conditions, with subscripts $i, j=1,2$, and 3 and $\mu, \lambda=1,2,3,4,5$, and 6.

c. Frequency constant and elastic constant. Frequency constants are useful parameters allowing the end user to determine the piezoelectric element dimensions to achieve a desired resonance frequency. ${ }^{17}$ Also, from the frequency constants, the phase velocities and elastic constants can be calculated. The frequency constant is related to the resonance (or antiresonance) frequency and the controlling dimension of the piezoelectric element, as given in the following equations:

$$
N_{r}=f_{r} \cdot l \text { or } N_{a}=f_{a} \cdot l .
$$

All solids deform under mechanical stress. Under small stresses, the strain is related to stress by Hooke's law, through the elastic compliance $s$ or elastic stiffness $c$. The elastic constants are closely related to the density $\rho$ and phase velocity $v$ (two times the frequency constant) of the piezoelectric element. The elastic constants can be determined following:

$$
s=1 / \rho v^{2} \text { or } c=\rho v^{2} .
$$

d. Acoustic impedance. The acoustic impedance $Z$ of a material, evaluating the propagation of acoustic waves across 
interfaces between different media, is also related to the elastic constant and/or phase velocity, following the relationship:

$$
Z=\sqrt{\rho c}=\rho v
$$

$\mathrm{Z}$ is an important parameter in piezoelectric applications related to acoustic waves. ${ }^{17}$ Analogous to electrical impedance, the transfer of acoustic energy from one medium to another will be maximized when the two media have the same acoustic impedance. For example, the acoustic impedance is about 30MRayl for relaxor-PT crystals, while it is only $1.5 \mathrm{MRayl}$ for water, thus, matching layers with intermediate acoustic impedances are desired to improve the acoustic energy transfer.

e. Electromechanical coupling. The electromechanical coupling factor $k_{i j}$ of a piezoelectric material refers to the conversion ability between electric and mechanical energy and vice versa, being closely related to the bandwidth of resonant devices. The relationship of electromechanical coupling and the piezoelectric coefficients follows the formulae: ${ }^{19,20}$

$$
k_{i \mu}^{2}=\frac{d_{i \mu}^{2}}{\varepsilon_{i i}^{T} s_{\mu \mu}^{E}}=\frac{e_{i \mu}^{2}}{\varepsilon_{i i}^{S} c_{\mu \mu}^{D}}=\frac{g_{i \mu}^{2}}{\beta_{i i}^{T} s_{\mu \mu}^{E}}=\frac{h_{i \mu}^{2}}{\beta_{i i}^{S} c_{\mu \mu}^{D}} .
$$

Electromechanical coupling factor is a dimensionless parameter relating to different vibration modes, since the mechanical energy of the piezoelectric element depends on the element dimension and vibration. The coupling can be calculated directly from the resonance and antiresonance frequencies measured on the piezoelectric elements, following:

$$
k^{2}=\frac{\pi f_{r}}{2} \frac{\pi}{f_{a}} \cot \left(\frac{\pi f_{r}}{2} \frac{k_{a}}{f_{a}}\right) \text { or } \frac{k^{2}}{k^{2}-1}=\frac{\pi f_{a}}{2} \frac{\pi}{f_{r}} \cot \left(\frac{\pi f_{a}}{2} \frac{f_{r}}{f}\right) .
$$

f. Mechanical quality factor. Analogous to the dielectric loss $\tan \delta_{e}$, mechanical loss $\tan \delta_{m}$ (inverse of the mechanical quality factor $Q_{m}$ ) describes the power loss over the energy stored in the system. For a piezoelectric resonator, the mechanical quality factor $Q_{m}$ characterizes the resonator's bandwidth relative to its center frequency, following:

$$
Q_{m}=f_{r} / \Delta f
$$

where $\Delta f$ is the frequency difference at $-3 \mathrm{~dB}$ of the maximum admittance. From a power dissipation viewpoint, high $Q_{m}$ is important in resonant applications, while high $Q_{e}$ (inverse of dielectric loss) is crucial for devices operating off-resonance.

For ferroelectric single crystals, the existence of domain wall motion/polarization rotation will significantly decrease $Q_{m}$. Other factors affecting the $Q_{m}$ include the polarization rotation angle, domain state (single domain vs multidomains), and domain size. External conditions will also affect the measured $Q_{m}$ values, such as measurement frequency, contact resistance (sample surface finish and adhesion of the electrode), and damping (for example, the $Q_{m}$ value meas- ured in air is much higher than the measured value in water, but lower compared to the value measured in vacuum).

\section{Ferroelectricity and related phenomena}

Ferroelectricity is the presence of a spontaneous polarization in a material, which can be reoriented between two or more distinct crystallographic directions by applying an external electric field. ${ }^{26-28}$ Ferroelectricity was discovered by Valasek ${ }^{29}$ in Rochelle salt in 1921, following which, ferroelectric behavior was observed in many families of materials, including hydrogen bonded crystals, complex oxides, polymers, and liquid crystals. Among oxides, materials with perovskite, tungsten bronze, and Aurivillius structures have been the mainstay for the ferroelectric studies. It should be noted that polarization reorientation can be induced by either applied stress (ferroelastic switching) or electric field (ferroelectric switching).

a. Ferroelectric domains and domain walls. A polarization develops once a ferroelectric crystal is cooled through Curie temperature, from the prototype paraelectric to ferroelectric phase with lower symmetry. Regions with uniform polarization are called ferroelectric domains, ranging in scale from few nanometers to few tens micrometers. The interface between two domains is called the domain wall, which is thin in order to reduce the elastic energy generated by the strain near the walls, being on the scale of a few lattice cells. In a ferroelectric crystal, the variety of domain patterns and the number of types of domain walls depend on the number of conceivable orientations of the dipole moment when the spontaneous polarization occurs from the prototype phase. ${ }^{30-35}$ For the tetragonal (T) case, domains can have their polarization along one of the six $\langle 001\rangle$ directions. Domain walls can lie in the (001) and (10) planes. The (001) walls are referred to as $180^{\circ}$ walls, since they separate domains which are $180^{\circ}$ to each other (antiparallel polarizations but with strain tensors that are necessarily

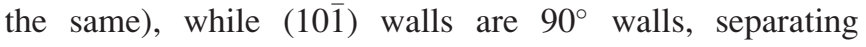
domains perpendicular to each other. For a rhombohedral (R) phase, the spontaneous polarization lies along one of the eight $\langle 111\rangle$ directions, thus both $180^{\circ}$ and $71^{\circ} / 109^{\circ}$ domain walls can occur. For the orthorhombic (O) phase, $180^{\circ}, 60^{\circ}$, $90^{\circ}$, and $120^{\circ}$ domain walls may exist, with spontaneous polarization lies along one of twelve $\langle 011\rangle$ directions. The switching of $180^{\circ}$ domain walls does not involve elastic deformation (so called ferroelectric domain walls), while the switching of non- $180^{\circ}$ walls involves elastic deformation, referred to as ferroelastic domain walls. Thus, both $180^{\circ}$ and non- $180^{\circ}$ domain walls may reduce the effects of depolarizing electric fields, but only the formation of non- $180^{\circ}$ domain walls can minimize the elastic energy. It is thought that the dielectric loss is related to the $180^{\circ}$ and non $-180^{\circ}$ domain wall motion under an applied electric field, while mechanical loss is only associated with non- $180^{\circ}$ domain wall motion under an applied stress. Domains can be observed using polarized light microscopy (PLM), scanning force microscopy, scanning electron microscopy, piezoresponse force microscopy, etc. 


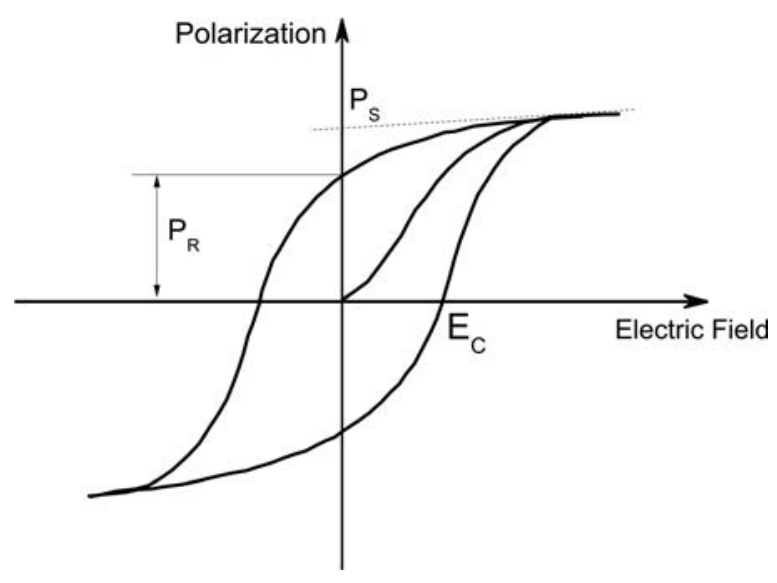

FIG. 1. A typical polarization hysteresis loop in ferroelectrics.

b. Ferroelectric hysteresis loop. Ferroelectric materials can be characterized by measuring their polarization $\mathrm{P}$ (D) as function of applied $a c$ electric field $(E)$, i.e., $P-E$ hysteresis loop. ${ }^{19,20,33-36}$ Ferroelectrics are strongly nonlinear in their dielectric behavior, thus, the variation of polarization with electric field is generally hysteretic as a consequence of domain wall motion. As shown in Figure 1, at low electric field, a linear relationship between $P$ and $E$ is observed, since the field is not sufficient to switch any domains and the crystal behaves as a normal dielectric. As the applied field increases, domains switch to the directions being more inclined to the electric field and the polarization increases rapidly until all the domains have switched, whereupon the polarization becomes saturated and relates to the spontaneous polarization $P_{S}$. As the field strength decreases to zero, some of the domains will remain aligned in the positive direction and the crystal will exhibit a remnant polarization $P_{R}$ (poled state). The remnant polarization cannot be removed until an applied field along negative direction reaches a certain value, defined as the coercive field $\mathrm{E}_{\mathrm{C}}$. An ideal hysteresis loop is symmetrical, with the positive and negative values of $E_{C}$ and $P_{R}$ being equal. The levels of $E_{C}, P_{S}, P_{R}$, and the squareness of the loop may be affected by many factors, including sample thickness, presence of charged defects (defect dipoles), mechanical stress, thermal treatment, measurement frequency, and space charge, etc.

Due to the existence of ferroelectric domains, the overall symmetry for nonpoled ferroelectrics is centro-symmetric and a piezoelectric response cannot be observed. Ferroelectric materials, thus, can be piezoelectrically active through the poling process, where an electric field is applied to switch the polarization vector of each domain inclining to the direction of the applied field. Generally, ferroelectric materials can be poled at $\sim 2 E_{C}$ electric field. In order to enhance poling efficiency and avoid cracking, ferroelectric crystals and polycrystalline ceramics are poled at elevated temperature.

c. Polymorphotropic phase transitions and morphotropic phase boundary (MPB). Phase transitions are major characteristics of most ferroelectrics. The most important transitions are those between the ferroelectric and paraelectric phases, and the temperature at which the transition occurs is called the Curie point (Curie temperature). ${ }^{19,20,33-36}$ Upon cooling through a Curie point, the crystallographic structure of a ferroelectric crystal is associated with a small distortion of the paraelectric structure, so the lattice symmetry of the ferroelectric phase is lower than that of the paraelectric phase. Ferroelectrics are also subject to transitions in which the crystallographic orientation of the polar axis changes, referring to polymorphic phase transitions, such as tetragonal to orthorhombic $\left(\mathrm{T}_{\mathrm{OT}}\right)$ and orthorhombic to rhombohedral $\left(\mathrm{T}_{\mathrm{RO}}\right)$ phase transitions in relaxorPT crystals. The thermodynamic properties (including the dielectric and piezoelectric) show anomalies and reach peak values when the temperature is in the vicinity of polymorphic phase transitions. Phase transitions can occur not only with changing temperature, but also under mechanical stress and/or electric field, generally, a positive electric field and uniaxial tension favor ferroelectricity. ${ }^{36}$

It is important to note that many ferroelectric solid solutions are found to possess an MPB, an abrupt structural change within a solid solution with composition, ${ }^{1}$ separating two ferroelectric phases of different crystallographic symmetries. For compositions near the MPB, the polarization can be easily rotated between different symmetries, giving rise to enhanced dielectric and piezoelectric properties. It should be noted here, both polymorphic phase transitions and MPBs lead to improved dielectric and piezoelectric properties in ferroelectric systems. However, the property enhancements at polymorphic phase transitions are strongly temperature dependent. $^{37,38}$

d. Domain engineering. As previously stated, the number and possible orientation of domains (domain configuration) are determined by the crystal symmetry, which are closely related to their dielectric and piezoelectric properties. The technique to control the desirable domain configuration is called domain engineering. Definition of a domain engineered structure is given as: "A domain engineered ferroelectric crystal is one, which has been poled by the application of a sufficiently large field along one of the possible polar axes of the crystal other than the zero-field polar axis, creating a set of domains in which the polarization vectors are oriented so that their angles to the poling direction are minimized." $33,39,40$

It was reported that the electric field induced hysteresisfree strain response in [001] oriented R relaxor-PT single crystals is related to the "domain engineered configuration." 3 In $\mathrm{R}$ crystals, there are eight degenerate domain variants, with the spontaneous polarization (polar vector) along one of the $\langle 111\rangle$ directions. When poled along the non-polar axis [001], four of the eight domain variants will be energetically favored by the poling field, with polar vectors along [111], [11̄i], [11]1], and [111]. Thus, four domain variants exist after the poling process, with polar vectors equally inclined to the poling direction $\left(\sim 54.7^{\circ}\right)$, exhibiting a macroscopic $4 \mathrm{~mm}$ symmetry. The resultant domain engineered configuration, labeled "4R," will be stable upon further application of an electric field along the poling direction, since there is no driving force for domain wall motion, and only the polar vectors in each domain variant will rotate towards to the direction of the 
applied field (polarization rotation or inclination). ${ }^{33}$ This accounts for the hysteresis-free strain characteristics and low dielectric loss observed in relaxor-PT crystals. An engineered domain configuration is basically multidomain, various domain engineered configurations can be realized depending on the crystal symmetry (phase) and the crystallographic orientation. For example, domain engineered configurations " $2 \mathrm{R}$ " and " $4 \mathrm{O}$ " can be formed when poled along the crystallographic [011] and [001] directions of $\mathrm{R}$ and $\mathrm{O}$ phase crystals, with macroscopic $\mathrm{mm} 2$ and $4 \mathrm{~mm}$ symmetries, respectively, as summarized in Table I. ${ }^{33,40}$ It should be noted that single domain states can be formed upon the application of electric field along spontaneous polarization directions, for example, $\langle 111\rangle$ for R crystal and $\langle 001\rangle$ for T crystal, leading to " $1 \mathrm{R}$ " and "1 T" states, respectively, though the single domain states are not generally stable, with the domains partially switching back after the removal of external electric field.

In engineered domain configurations, relaxor-PT crystals are found to possess high piezoelectric coefficients, owing to the easy tilt of spontaneous polarization by applying electric field along non-polar directions. Other approaches, such as domain wall engineering using patterning electrodes or special poling process, are also found to increase the piezoelectric activity with increasing domain wall densities (decreasing domain sizes) in $\mathrm{BaTiO}_{3}$ or $\mathrm{PMN}-$ PT crystals, ${ }^{30,41-43}$ due to the extrinsic contribution from the domain wall motion.

e. Aging behavior and piezoelectric nonlinearity. The domain alignment during the poling process leaves the ferroelectric material in a state of high internal stress due to strain accommodations associated with the ferroelastic domain wall reversal/polarization rotation. The gradual relief of various stresses by domain realignment, usually in small steps as time elapses, gives rise to the observed change in properties with time. For example, the dielectric and piezoelectric coefficients decrease while the mechanical and electrical quality factors increase, as a logarithmic function of time. ${ }^{17,19,20,36}$ In order to obtain ferroelectric materials with stable properties, accelerated aging treatments have been used to relieve these interdomain stresses.

Another effect associated with multidomain ferroelectrics is piezoelectric nonlinearity. Deviations from linear behavior occur in ferroelectrics due to the domain wall motion/domain switching, which are related to dissipation and hysteresis. ${ }^{36}$ Under low electrical or mechanical drive fields, ferroelectric materials may be considered linear, where the dielectric and piezoelectric constants are attributed to intrinsic contribution and reversible domain wall motion. With increasing electrical or mechanical drive, there is a disproportionate increase in dielectric and piezoelectric response, being related to irreversible domain wall motion. Other phenomena, such as field/mechanical stress induced phase transitions and cyclic electric fatigue, are also related to domain effects. More details about piezoelectric nonlinearity can be found in the reference by Damjanovic. ${ }^{36}$

\section{Background on pervoskite ferroelectric materials}

\section{History of pervoskite ferroelectric ceramics}

A breakthrough in the discovery of ferroelectric materials occurred in mixed-oxide perovskites with the general formula $\mathrm{ABO}_{3}$. Milestones included barium titanate $\left(\mathrm{BaTiO}_{3}\right)$ polycrystalline ceramics in the late 1940 s (Refs. 44-46) and PZT solid solutions in the early $1950 \mathrm{~s},{ }^{1,47-49}$ which have since dominated the piezoelectric ceramic markets. The major interest is that large dielectric and piezoelectric properties are achieved in PZT with compositions close to an MPB. In addition, PZT ceramics can be modified with different dopants, exhibiting "hard" characteristics with acceptor dopants or "soft" behavior with donor dopants, suitable for a broad range of electromechanical applications. However, it is hard to grow PZT single crystals in usable size, due to the incongruent melting behavior. In late $1950 \mathrm{~s}$, unusual dielectric behavior was reported in complex perovskites with chemical formula of $\mathrm{Pb}\left(\mathrm{B}^{\prime}, \mathrm{B}^{\prime \prime}\right) \mathrm{O}_{3}$, where $\mathrm{B}$ ' is a low valence cation and $\mathrm{B}$ " is a high valence cation, later named relaxor ferroelectrics. ${ }^{50}$ Materials with this chemical structure exhibit frequency-dispersed dielectric permittivity. Of the many interesting relaxor materials discovered, lead magnesium niobate (PMN) was the most promising, with high dielectric permittivities and a strong nonhysteretic electrostrictive effect at room temperature. ${ }^{34,51,52}$ In the late $1970 \mathrm{~s}$, it was discovered that by creating a solid solution of PMN with addition of PT, the Curie point and the nonhysteretic quadratic strains increased. ${ }^{53}$ This finding was quickly followed by the discovery that with increased compositional levels of PT, the electrostrictive behavior of PMN-PT was replaced by a more "classical" ferroelectric behavior, leading to the mapping of the PMN-PT binary phase diagram, which

TABLE I. Domain engineered configuration in Relaxor-PT single crystal systems (Refs. 33 and 40).

\begin{tabular}{|c|c|c|c|c|c|}
\hline Crystal phase & Polar directions & Poling direction & Engineered domain configuration & Macroscopic symmetry & Exist domain walls \\
\hline \multirow[t]{3}{*}{ Rhombohedral } & \multirow[t]{3}{*}{$\langle 111\rangle$} & [001] & $4 \mathrm{R}$ & $4 \mathrm{~mm}$ & $109^{\circ}, 71^{\circ}$ \\
\hline & & [011] & $2 \mathrm{R}$ & $\mathrm{mm} 2$ & $71^{\circ}$ \\
\hline & & [111] & $1 \mathrm{R}$ & $3 \mathrm{~m}$ & Single domain \\
\hline \multirow[t]{3}{*}{ Orthorhombic } & \multirow[t]{3}{*}{$\langle 011\rangle$} & [001] & 40 & $4 \mathrm{~mm}$ & $90^{\circ}$ \\
\hline & & [011] & 10 & $\mathrm{~mm} 2$ & Single domain \\
\hline & & [111] & 30 & $3 \mathrm{~m}$ & $60^{\circ}$ \\
\hline \multirow[t]{3}{*}{ Tetragonal } & \multirow[t]{3}{*}{$\langle 001\rangle$} & {$[001]$} & $1 \mathrm{~T}$ & $4 \mathrm{~mm}$ & Single domain \\
\hline & & [011] & $2 \mathrm{~T}$ & $\mathrm{~mm} 2$ & $90^{\circ}$ \\
\hline & & [111] & $3 \mathrm{~T}$ & $3 \mathrm{~m}$ & $90^{\circ}$ \\
\hline
\end{tabular}


showed an MPB separating rhombohedral and tetragonal phases. ${ }^{54,55}$ Analogous to PZT, the piezoelectric properties of PMN-PT ceramics reach peak values at the MPB, being on the order of $700 \mathrm{pC} / \mathrm{N}^{55}$ In 1982, a new method, called two-step precursor process, for synthesis of PMN ceramics and other complex mixed oxide ceramics was reported by Swartz and Shrout, ${ }^{56}$ greatly decreased the presence of unwanted pyrochlore phase.

\section{Relaxor-PT single crystals}

Although the growth of relaxor PMN crystals was reported in the early $1960 \mathrm{~s}$, it was not until the early $1970 \mathrm{~s}$ that single crystal relaxor ferroelectrics emerged, specifically lead zinc niobate $\left(\mathrm{Pb}\left(\mathrm{Zn}_{1 / 3} \mathrm{Nb}_{2 / 3}\right) \mathrm{O}_{3}-\mathrm{PZN}\right){ }^{34}$ It was found that these crystals could be readily grown from the high temperature $\mathrm{PbO}$ flux. The solid solution was found to possess an MPB at around 9\% $\mathrm{PT}$, with $d_{33}>1500 \mathrm{pC} / \mathrm{N}$ reported for [001] poled PZN-PT crystals. The high piezoelectric property was explained by a strong crystal anisotropy in single crystals. ${ }^{57,58}$ This work was followed by the investigation on single crystal PMN-PT in 1989 s. ${ }^{59,60}$ The piezoelectric properties of PMN-PT and PZN-PT single crystals poled along different crystallographic directions were reported in late $1990 \mathrm{~s}$ and early $2000 \mathrm{~s},{ }^{3,4,61-75}$ showing ultrahigh piezoelectric coefficients and electromechanical coupling factors, being on the order of $>2000 \mathrm{pC} / \mathrm{N}$ and $>0.9$, far outperforming state-of-art PZT ceramics, as shown in Figure 2. Today, relaxor ferroelectrics PMN-PT single crystals continue to be an exciting research area that promises even further discoveries and have been recently commercialized using the Bridgman crystal growth method. ${ }^{34,76}$

Implementation of these crystals has yet to meet expectation, due to their low Curie temperature $T_{C}$ and ferroelectric phase transition temperature $T_{R T}$. The temperature stability and long term reliability of single crystal devices are a major concern for many applications. In addition to the low $T_{C} / T_{R T}$, the low $E_{C}$ brings up the issues of polarization stability under various storage and driving conditions. For this case, a $d c$ bias may be required to maintain the polarization and the performance of the devices. Loss in sensitivity, however, occurs when applying $d c$ bias, which also adds complexity and the cost to the driving electronics. Furthermore, though having low dielectric loss, being on the order of 0.004 , their low mechanical quality factors $Q_{m}$, being $\sim 100$, limit their use in resonance based applications, such as high power transducers and transformers. Owing to the above mentioned issues observed in PMN-PT and PZN-PT crystals, new single crystal systems with broad temperature usage range and improved reliability under the thermal/electric field/mechanical stress are desired. ${ }^{76}$

Over the last $10 \mathrm{yr}$, extensive effort has been focused on new crystals with high $\mathrm{T}_{\mathrm{C}} / \mathrm{T}_{\mathrm{RT}}$, including binary systems $\mathrm{Pb}\left(\mathrm{Sc}_{0.5} \mathrm{Nb}_{0.5}\right) \mathrm{O}_{3}-\mathrm{PbTiO}_{3}$ (PSN-PT), ${ }^{77-83} \mathrm{~Pb}\left(\mathrm{In}_{0.5} \mathrm{Nb}_{0.5}\right) \mathrm{O}_{3}-$ $\mathrm{PbTiO}_{3}$ (PIN-PT), ${ }^{84-89} \mathrm{~Pb}\left(\mathrm{Yb}_{0.5} \mathrm{Nb}_{0.5}\right) \mathrm{O}_{3}-\mathrm{PbTiO}_{3}$ (PYN$\mathrm{PT}),{ }^{90-94} \mathrm{BiScO}_{3}-\mathrm{PbTiO}_{3}$ (BSPT) ${ }^{95-101}$ and ternary systems $\mathrm{Pb}\left(\mathrm{Sc}_{0.5} \mathrm{Nb}_{0.5}\right) \mathrm{O}_{3}-\mathrm{Pb}\left(\mathrm{Mg}_{1 / 3} \mathrm{Nb}_{2 / 3}\right) \mathrm{O}_{3}-\mathrm{PbTiO}_{3}$ (PSNPMN-PT $),{ }^{102} \mathrm{~Pb}\left(\mathrm{In}_{0.5} \mathrm{Nb}_{0.5}\right) \mathrm{O}_{3}-\mathrm{Pb}\left(\mathrm{Mg}_{1 / 3} \mathrm{Nb}_{2 / 3}\right) \mathrm{O}_{3}-\mathrm{PbTiO}_{3}$ (PIN-PMN-PT), ${ }^{103-126} \mathrm{~Pb}\left(\mathrm{Mg}_{1 / 3} \mathrm{Nb}_{2 / 3}\right) \mathrm{O}_{3}-\mathrm{PbZrO}_{3}-\mathrm{PbTiO}_{3}$
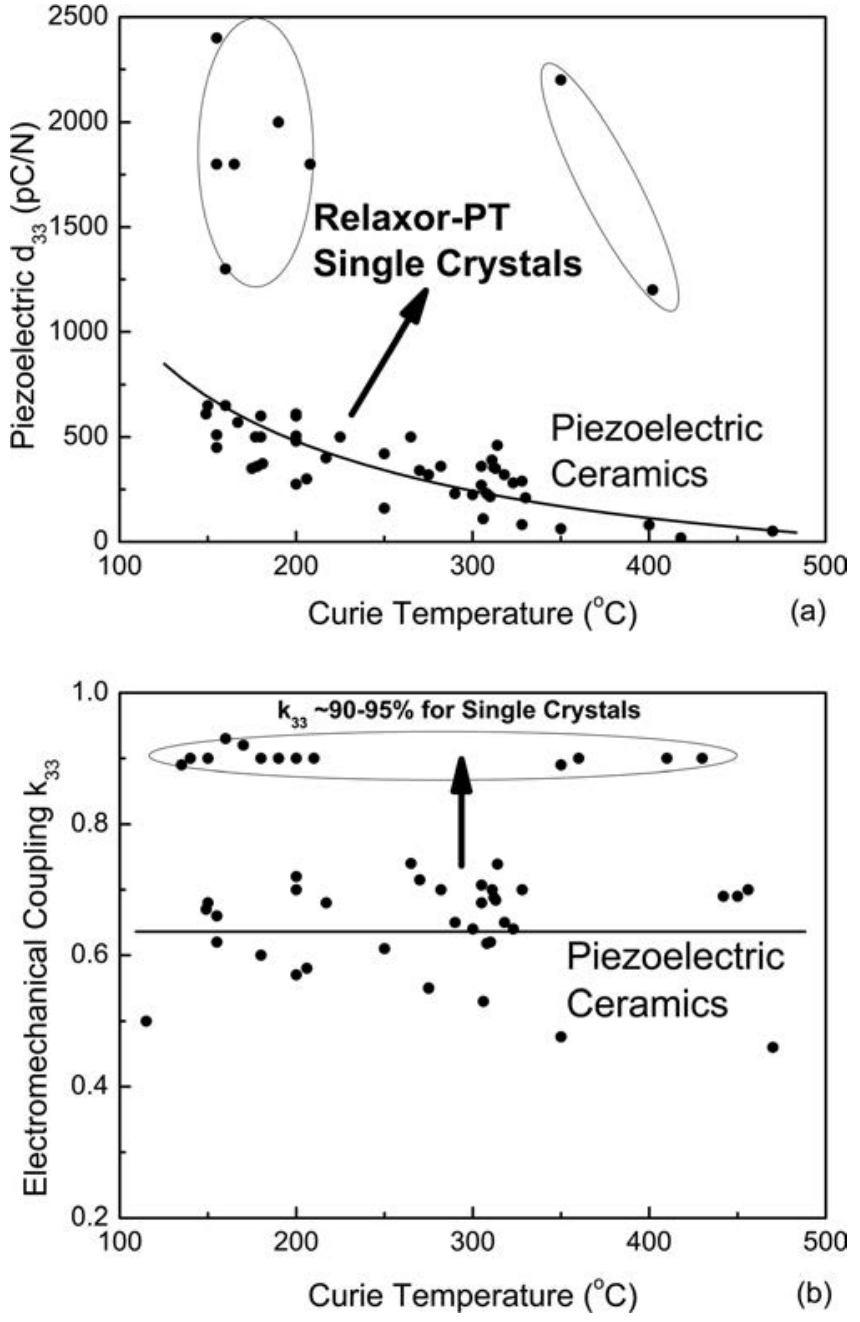

FIG. 2. Piezoelectric $d_{33}$ (a) and electromechanical $k_{33}$ (b) of relaxor-PT crystals, compared to polycrystalline ceramics as function of Curie temperature (data are from Ref. 76).

(PMN-PZT), ${ }^{127-132}$ etc. Among those crystal systems, only PIN-PMN-PT and PMN-PZT systems have been found to be grown in large size and high quality. With these developments in relaxor-PT single crystals, the concept of various generation of crystals was proposed by Smith. ${ }^{133}$ First, generation crystals, e.g., PMN-PT and PZN-PT, exhibit high electromechanical coupling and piezoelectric coefficients that allow transducers to be fabricated with increased bandwidth $(\times 2-3)$, higher sensitivity $(+12 \mathrm{~dB})$ and higher source level $(+12 \mathrm{~dB})$ when compared with the state-of-art polycrystalline PZT technology, which already have been commercialized in medical ultrasonic transducers. Second generation crystals extend the high electromechanical properties to a broader range of temperature, electric field and mechanical stress, expanding their design envelope by reducing the need for heat shunts and applied $d c$ bias fields. Crystals with higher ferroelectric phase transition temperatures and higher coercive fields are in this category, where the potential commercialization of the ternary PIN-PMN-PT and PMN-PZT are expected. Third generation crystals include the addition of minor dopants to tailor the crystal's electromechanical parameters, analogous to PZTs. For example, Mn doped relaxor-PT crystals have been developed, with 
greatly increased mechanical quality factors, which will benefit the resonance based devices, such as sonar transducers, ultrasonic motors, or piezoelectric transformers.

Table II compiles the general properties for the three generations of crystal systems, where the $T_{C} / T_{R T}$ and $E_{C}$ of 2nd generation crystals were found to be higher when compared to their 1st generation crystal counterparts, with comparable dielectric and piezoelectric properties. As expected, the mechanical quality factor of 3 rd generation crystals was found to be $>5$ times higher than that of 1 st generation crystals, due to the existence of an internal bias, induced by $\mathrm{Mn}$ acceptor dopants. ${ }^{134-137}$

\section{SINGLE CRYSTAL GROWTH: ISSUES AND FUTURE DIRECTION}

Numerous crystal growth techniques have been developed to synthesize single crystals. These techniques include growth from high temperature solutions (flux; flux Bridgman; top seeding crystal growth; Kyropoulos), crystal growth from the solid state, crystal growth from the pure melt (Bridgman; Stockbarger; Czochralski; floating-zone melting, etc.); crystal growth from vapor (such as chemical vapor deposition and physical vapor transport); crystal growth from aqueous or nonaqueous solvent; hydrothermal; etc. ${ }^{138,139}$

As discussed in Sec. I C 2, three generations of relaxorPT single crystals have been developed. The successful growth of relaxor single crystals was first reported in the late $1950 \mathrm{~s}$ for $\mathrm{Pb}\left(\mathrm{Mg}_{1 / 3} \mathrm{Ta}_{2 / 3}\right) \mathrm{O}_{3}$ (PMT) and $\mathrm{Pb}\left(\mathrm{Zn}_{1 / 3} \mathrm{Nb}_{2 / 3}\right) \mathrm{O}_{3}$ (PZN) crystals using the high temperature solution growth technique with $\mathrm{PbO}$ as flux. ${ }^{140,141}$ In the period of 1960-1990, the growth of PZN-PT and PMN-PT single crystals was reported using $\mathrm{PbO}$ or $\mathrm{PbO} / \mathrm{B}_{2} \mathrm{O}_{3}$ as fluxes, but with limited success to obtain large size crystals. ${ }^{57-60,142-147}$ In the mid1990 s, systematic studies of flux grown PZN-PT single crystals were carried out, with crystal sizes being sufficient for detail property characterization and device exploration. The ultrahigh piezoelectric properties and the potential applications of the relaxor-PT single crystals drew extensive attention and renewed interest worldwide in growing large-size and commercially viable single crystals. $3,61,62,148-150$

In this section, crystal growth approaches related to relaxor-PT single crystals will be discussed, with emphasis on high temperature solution methods, Bridgman, and solid state conversion method.

\section{A. High temperature solution growth}

For the case of growth from a high temperature solution, the constituents of the material to be crystallized are dissolved in a suitable solvent and crystallization occurs as the solution becomes critically supersaturated. The supersaturation may be promoted by evaporation of the solvent, by cooling the solution or by a transport process in which the solute is made to flow from a hotter to a cooler region. The advantage of using a solvent is that crystal growth occurs at a lower temperature than that required for growth from the melt, thus, this method is useful for growing crystals that are incongruently melting or phase instable, undergo a phase transition below the melting point which may result in severe straining or cracking, have a very high vapor pressure at the melting point or highly refractory materials. ${ }^{151}$ Relaxor-PT single crystals, such as PZN-PT, ${ }^{152-157}$ PYN-PT (Ref. 91) and BSPT, ${ }^{98,158}$ show incongruently melting characteristics and pyrochlore phase $\left(\mathrm{Pb}_{2} \mathrm{Nb}_{2} \mathrm{O}_{7}\right)$ at high temperature. The size of relaxor-PT single crystals produced by the conventional flux growth technique remains relatively small. In order to grow large PZN-PT single crystals, other flux growth techniques have been explored. These include the solution Bridgman ${ }^{159-170}$ and top-seeded solution growth (TSSG) ${ }^{171-175}$ techniques, where PZN-PT single crystals with $75 \mathrm{~mm}$ in diameter have been produced. In this section, the development and status of PZN-PT single crystals growth using high temperature solution method will be discussed.

\section{Conventional flux method}

Figure 3 shows a typical experimental set-up for PZNPT single crystal growth using conventional flux method. The components, including the nominal crystal composition

TABLE II. The property comparison of various generation relaxor-PT single crystals.

\begin{tabular}{|c|c|c|c|c|c|c|c|c|c|}
\hline Crystal & $\mathrm{T}_{\mathrm{C}}\left({ }^{\circ} \mathrm{C}\right)$ & $\mathrm{T}_{\mathrm{RT}}\left({ }^{\circ} \mathrm{C}\right)$ & $\mathrm{E}_{\mathrm{C}}(\mathrm{kV} / \mathrm{cm})$ & $\mathrm{E}_{\text {int }}(\mathrm{kV} / \mathrm{cm})$ & $\varepsilon_{\mathrm{r}}$ & $d_{33}(\mathrm{pC} / \mathrm{N})$ & $k_{33}$ & $Q_{\mathrm{m}}$ & References \\
\hline \multicolumn{10}{|c|}{ First generation crystals } \\
\hline PMN-0.29PT & 135 & 96 & 2.3 & - & 5400 & 1540 & 0.91 & 150 & 241 \\
\hline PMN-0.33PT & 155 & 65 & 2.8 & - & 8200 & 2800 & 0.95 & 100 & 31 \\
\hline \multicolumn{10}{|c|}{ Second generation crystals } \\
\hline PIN-PMN-PT & 191 & 125 & 5.0 & - & 4400 & 1510 & 0.92 & 180 & 109 \\
\hline PIN-PMN-PT (MPB) & 197 & 96 & 5.5 & - & 7240 & 2740 & 0.95 & 120 & 110 \\
\hline PMN-PZT & 210 & 113 & 5.0 & - & 5000 & 1750 & 0.92 & 150 & 130 \\
\hline PMN-PZT & 216 & 144 & 4.6 & - & 4850 & 1530 & 0.93 & 100 & 127 \\
\hline BSPT57 & 402 & 349 & 13.7 & - & 3000 & 1150 & 0.91 & - & 97,101 \\
\hline PYN-0.45PT & 325 & 160 & 12.5 & - & 2000 & $2000^{\mathrm{a}}$ & 0.90 & - & 76 \\
\hline \multicolumn{10}{|c|}{ Third generation crystals } \\
\hline Mn:PIN-PMN-PT & 193 & 119 & 6.0 & 1.0 & 3700 & 1120 & 0.90 & 810 & - \\
\hline Mn:PMN-PZT & 203 & 141 & 6.3 & 1.6 & 3410 & 1140 & 0.92 & 1050 & 136 \\
\hline
\end{tabular}

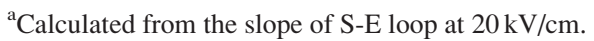


and flux, are mixed thoroughly and charged into the platinum (Pt) crucible, and then the Pt crucible is covered with a lid and placed into a sealed alumina crucible, which can reduce the evaporation of lead at high temperature. The assembly is placed in a muffled resistant heating furnace and allowed to heat to temperature in the range of $1200-1250^{\circ} \mathrm{C}$, with a dwell time of several hours to stabilize and homogenize the melt. Subsequently, the assembly is cooled at a controlled cooling rate, typically in the range of $0.5-1{ }^{\circ} \mathrm{C} / \mathrm{min}$, to initiate the growth process. The growth process is allowed to end at temperature of $800-1000^{\circ} \mathrm{C}$, then cooled to room temperature at a rate of $50^{\circ} \mathrm{C} / \mathrm{h}$. In order to eliminate or reduce points of spontaneous nucleation, a local cooling arrangement, such as thin metal rod or metal wire, or controlled oxygen gas flow, is used at the bottom center of the Pt crucible. The thermal gradient is usually about $10^{\circ} \mathrm{C} / \mathrm{cm}$ at the cooling spot. Though a cooling spot is used, the thermal gradient for flux method is very small, the crystal is not exposed to steep temperature gradients, and the crystal can grow in an unconstrained fashion, together with the relatively low growth temperature, leading to an improved crystal quality with respect to point defects, dislocation densities, and low angle grain boundaries, compared to crystals grown directly from melt. Disadvantages of this method, however, are substitutional or interstitial incorporation of solvent ions into the crystal lattice, microscopic, or macroscopic inclusions of solvent, impurities, or non-uniform doping. ${ }^{139,146-151}$

For the high temperature solution method, the selection of flux is very important. $\mathrm{PbO}$ or $\mathrm{PbO}-\mathrm{B}_{2} \mathrm{O}_{3}$ mixtures are commonly used in the growth of relaxor-PT single crystals. A $\mathrm{PbO}$ melt has a reasonably low viscosity and high solubility for complex oxides, with a melting temperature of $886^{\circ} \mathrm{C}$. It will form an eutectic compound with PZN-PT at $828^{\circ} \mathrm{C}$, with composition of $25 \mathrm{wt}$ \% (PZN-0.09PT)/75 wt. $\%$ PbO. ${ }^{157}$ PZN-PT crystals will partially decompose into a pyrochlore phase above $1140^{\circ} \mathrm{C}$ and incongruently melt at $1225+2{ }^{\circ} \mathrm{C}$, thus, crystallization temperatures are selected to below this temperature. Typical solute-to-flux weight ratios used are in the range of $0.65: 0.35$ to $0.5: 0.5$, which

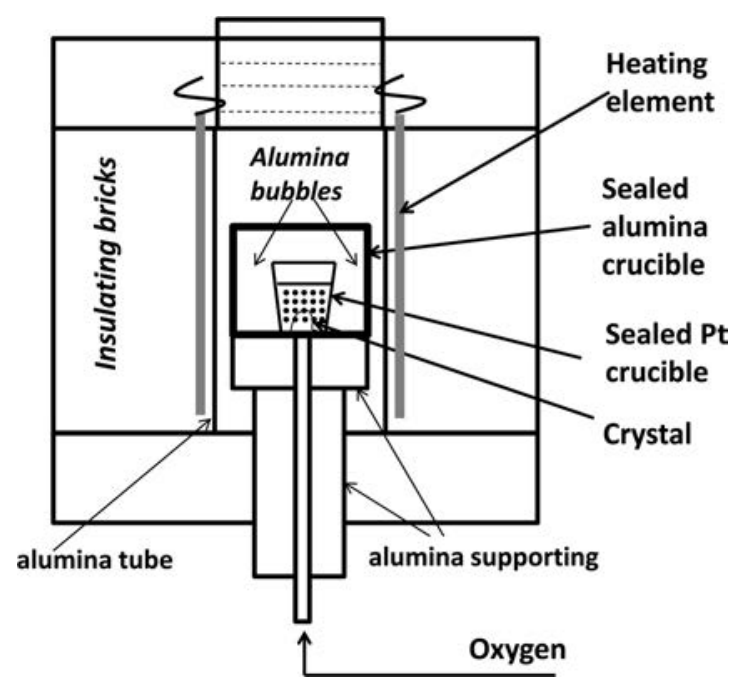

FIG. 3. Schematic experimental set-up of the PZN-PT crystal growth from high temperature solution. guarantee a crystal growth temperature below $1140^{\circ} \mathrm{C}$. After the growth process, the crystals are leached out of the flux using hot nitric acid. The growth of PZN-PT crystals starts by nucleation at [111] corners followed by spreading down adjacent [001] edges and (001) crystal faces at a low supersaturation in the solution, while the growth is dominated via nucleation at [001] edges and on (001) faces when the degree of supersaturation increase. It was reported that by engineering the isotherms in the solution, [111] corner nucleation is controlled, while (001) layer growth is promoted. The crystal growth process in this case is dominated by layer growth on certain (001) crystal planes. ${ }^{147}$ Each (001) layer represents a layer of material grown within a given time interval, reducing solute segregation, thus, wafers cut parallel to the prevalent (001) layer growth plane show improved compositional homogeneity. The crystal growth occurs in an equilibrium or near-equilibrium condition, such as controlled cooling/ growth rates, compositional uniformity between wafers can also be improved. ${ }^{147}$ Figure 4 shows large size PZN-PT single crystals ( $\geq 35 \mathrm{~mm}$ edge length) grown using the above scheme at Microfine Materials Technologies, ${ }^{159}$ with translucent and light yellow to brownish-yellow in color while exhibiting prominent (001) habitual facets. The obtained crystals show improved compositional uniformity, with a Curie temperature variation being within $\pm 2{ }^{\circ} \mathrm{C}$, corresponding to the PT content variation less than $\pm 0.5 \%$ in the whole ingot.

\section{Flux Bridgman}

Bridgman method is useful for the production of single crystals, however, PZN-PT easily changes to pyrochlore phase at high temperature, with perovskite phase stable only in $\mathrm{PbO}$ flux, thus, modified solution Bridgman method has been applied to grow PZN-PT crystals. ${ }^{160-171}$ The PZN-PT compound with flux is charged in the Bridgman crucible, with the crucible sealed and buried into alumina powder in a refractory tube. Alumina powder is used to support the crucible as well as prevent $\mathrm{PbO}$ evaporation. An oxygen gas cooling system (with cooling rod) is designed and assembled at the bottom of the crucible to induce nucleation at the bottom of the crucible, providing a temperature gradient, usually on the order of $15-20^{\circ} \mathrm{C} / \mathrm{cm}$. The oxygen flow rate and the diameter of the rod are important to accurately control the thermal gradient. The hot zone temperature of the furnace is $>1250{ }^{\circ} \mathrm{C}$, with the crucible lowered through the thermal zones at a rate of $0.1-0.6 \mathrm{~mm} / \mathrm{h}$. Due to a lack of forced convection in the Bridgman growth, the growth rate is strongly dependent on mass transport. The crucible lowering rate is important, depending on the solute transport rate at the respective temperature range. Large size crystals have been reported up to $75 \mathrm{~mm}$ in diameter and $55 \mathrm{~mm}$ in length. ${ }^{160-164} \mathrm{PbO}$ flux inclusions were observed in the bottom part and the peripheral regions of the crystal, probably as the results of inadequate temperature gradient and the difference between the crucible lowering rate and the solute transport rate (crystal growth rate). In the solution Bridgman growth, no seed material is used to initiate nucleation. Depending on the growth condition, the growth direction 


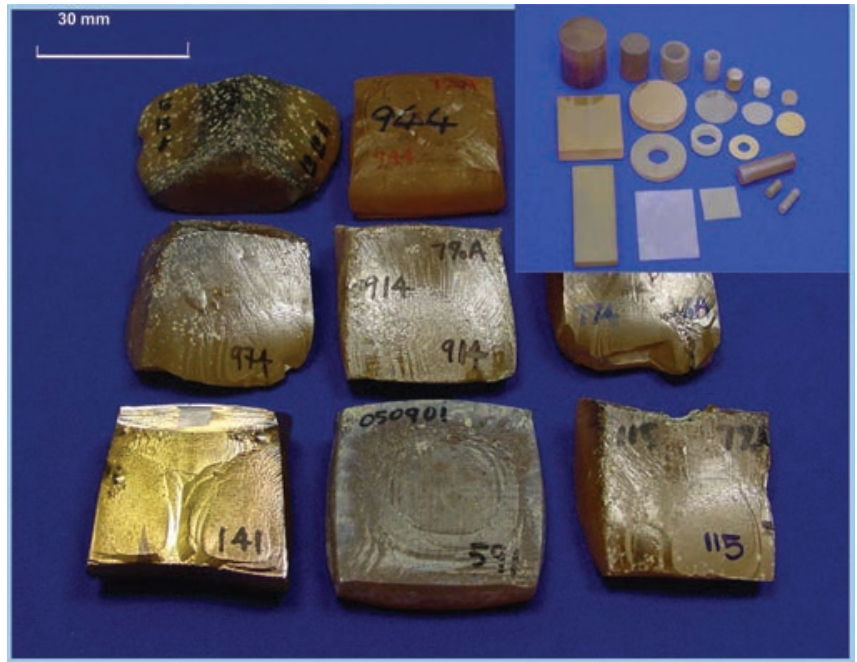

FIG. 4. (Color) Large PZN-PT single crystals grown from PbO flux (Courtesy of Dr. L. C. Lim from Microfine Materials Technologies). ${ }^{159}$

can vary from [111] to [100]. Large size PZN-PT crystals obtained show (100) habitual planes, due to the fact that growth rate along [111] is the highest, while [100] direction exhibit the lowest growth rate. The variation of $\mathrm{Ti}^{4+}$ concentration along the growth direction was found to be $\pm 0.6 \mathrm{~mol}$. $\%$ over a distance of about $20 \mathrm{~mm} .{ }^{160-164}$ Compositional uniformity of the as-grown PZN-PT crystals were reported to be greatly improved with much less inclusion defect, by using two-step solution Bridgman method. ${ }^{139,167-170}$ In this approach, PZN-PT seed crystal is achieved by spontaneous nucleation at the cooling spot (oxygen flow) in the first step. The temperature goes up due to the termination of oxygen flow at the end of the first step, which will melt the seed partially and re-establish a stable solid-liquid interface during the second growth step, thus, inclusions in the crystals are reduced.

Other high temperature solution growth methods, including top seeding solution growth (TSSG), ${ }^{172-176}$ have also been studied to grow PZN-PT single crystals. Crystals with $35 \mathrm{~mm}$ in diameter and $14 \mathrm{~mm}$ in length have been obtained. Compared to solution Bridgman, the TSSG technique offers the advantage of low cost, with ease of separating the primary grown crystals from the flux and extending life of Pt crucibles. Furthermore, TSSG can be effectively improved for growth of uniform crystals by continually charging the raw powders into the crucible to offset compositional variation in the melt during growth. Lead oxide evaporation and decomposition of the as-grown perovskite crystals during the crystal growth process, however, limit the TSSG method for large scale production. ${ }^{172,173}$

\section{B. Modified Bridgman}

Analogous to the Czochralski method, Bridgman method also grows single crystals directly from the melt, which is the most straightforward and economical way to grow high quality and large size crystals. ${ }^{138}$ The work by Bridgman was directed toward the growth of single crystals, where he used a vertically mounted tubular electric furnace to melt the charge and lowered the ampoule containing the charge through an axial thermal gradient, so the molten ingot is gradually crystallized from one end to the other. ${ }^{177}$ A similar vertical configuration was employed by Stockbarger to grow single crystals of lithium fluoride, using two furnaces mounted coaxially, which possess high temperature and low temperature zones. ${ }^{178}$ Horizontal directional solidification has also been widely employed and is known as "horizontal Bridgman" growth. Both horizontal and vertical directional solidification techniques (referred to as normal freezing) have been extended to melt partial charge, with a high temperature zone traversing along the charge, which is referred to as zone melting. The zone melting configuration has the advantage over the normal freezing method of avoiding macro-segregation in the crystals. ${ }^{138,179-182}$

PMN-PT single crystals have been grown from high temperature solution with $\mathrm{PbO}$ or $\mathrm{PbO} / \mathrm{B}_{2} \mathrm{O}_{3}$ as the flux, ${ }^{59,60,147,159}$ however, the growth rate and crystal size using the flux method are not suitable for commercial production. The first report on the melt growth of PMN-PT crystals using a modified Bridgman furnace occurred in 1997, suggesting that the Bridgman method could be used to grow PMN-PT crystals as an alternative to flux method, ${ }^{183,184}$ after which, Bridgman approach has been widely used to grow PMN-PT crystals. ${ }^{185-194}$ Currently, binary PMN-PT and ternary PIN-PMN-PT crystals are commercially manufactured along [001] direction, with 75-100 $\mathrm{mm}$ in diameter and 150$200 \mathrm{~mm}$ in length, by using modified multi-crucible Bridgman method, as shown in Figure 5. ${ }^{185,186,195-200}$

Figure 6 shows typical experimental set-up for Bridgman growth technique. Crystal growth is usually performed in either straight or tapered $\mathrm{Pt}$ crucibles. After oriented PMN-PT crystal seed and the sintered pellets charged into the $\mathrm{Pt}$ crucible, it is sealed with a $\mathrm{Pt}$ lid to prevent the $\mathrm{PbO}$ evaporation at high temperature. The crucible is placed into alumina buffer tube and the gap between the crucible and the alumina tube is filled with alumina or zirconia powders. ${ }^{185}$

\section{[001] grown PMN-PT \& PIN-PMN-PT (Ф100mm)}
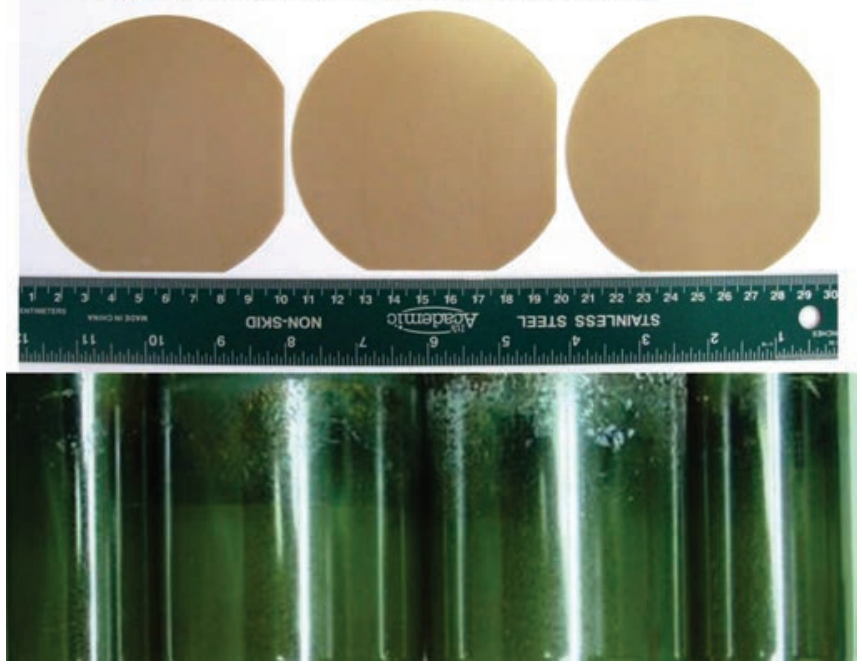

FIG. 5. (Color) PMN-PT and PIN-PMN-PT single crystals (100 mm diameter) grown by multi-crucible Bridgman method (courtesy of Dr. J. Luo from TRS Technologies). ${ }^{199}$ 


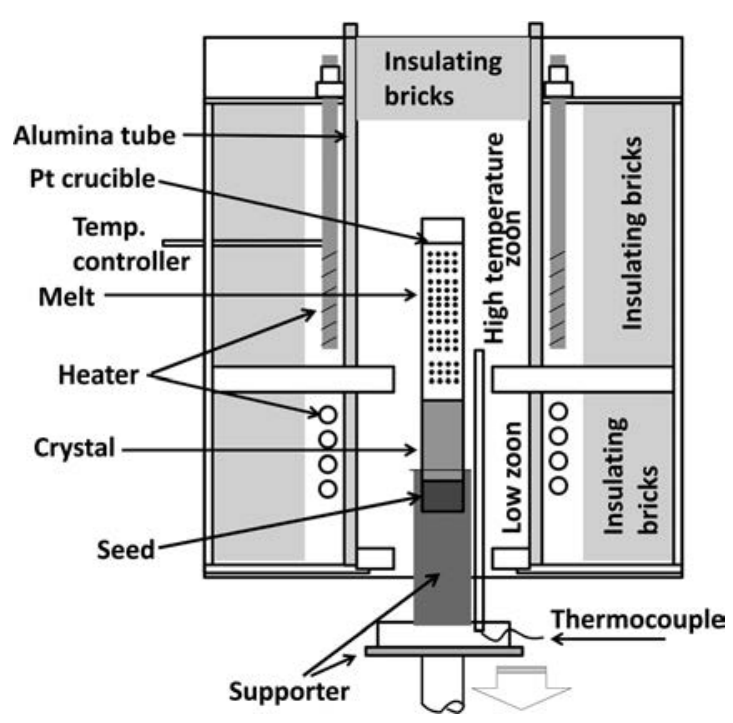

FIG. 6. Schematic experimental set-up of a Bridgman growth system.

The assembly is loaded into resistance-heating multi-zone furnaces with soaking temperature higher than $1350{ }^{\circ} \mathrm{C}$ and dwelling time of $5-10 \mathrm{~h}$. The axial temperature gradient is set to be larger than $20^{\circ} \mathrm{C} / \mathrm{cm}$ at the solid-liquid interface. Several thermocouples are used to monitor the melt temperatures during growth. ${ }^{186}$ Unidirectional solidification is accomplished by slowly lowering the Pt crucible through the temperature gradient with a translation speed less than $1 \mathrm{~mm} / \mathrm{h}$. After the growth process, the furnace is allowed to cool to room temperature at a rate of $50{ }^{\circ} \mathrm{C} / \mathrm{h}$ to prevent the cracking of the crystal. The crucibles are not reusable, as the grown crystals cannot be removed without cutting away the crucible. ${ }^{185-194}$

For PMN-PT crystal growth, owing to the large difference in melting point and density between each oxide, especially between $\mathrm{PbO}\left(\mathrm{T}_{\mathrm{m}}=886^{\circ} \mathrm{C}\right.$ and $\left.\rho=9.53 \mathrm{~g} / \mathrm{cm}^{3}\right)$ and $\operatorname{MgO}\left(\mathrm{T}_{\mathrm{m}}=2852{ }^{\circ} \mathrm{C}\right.$ and $\left.\rho=3.58 \mathrm{~g} / \mathrm{cm}^{3}\right)$, it is usually difficult to get a homogeneous perovskite phase by directly mixing the oxides without the presence of a minor amount of the pyrochlore phase. ${ }^{186}$ Pre-synthesized PMN-PT using a modified columbite precursor method has been proved to be the most efficient way to eliminate the pyrochlore phase. ${ }^{56}$ Powders with nominal compositions are calcined at $850^{\circ} \mathrm{C}$ for several hours to synthesize the PMN-PT compound, and then ground, mixed and pressed into pellets, subsequently sintered to reduce the volume of the pellets prior to the charge in $\mathrm{Pt}$ crucibles. During crystal growth, it is important to understand the high temperature phase equilibrium of the solid solution. The high temperature phase diagram of the PMN-PT system was reported in 2003, showing a typical binary solid solution behavior. ${ }^{193}$ Though PMN and PT are congruently melting compounds, the segregation coefficient of $\mathrm{Ti}$ is less than one, giving rise to compositional variation in the asgrown crystal boule. For electromechanical applications, single crystals with high property uniformity are desired. Due to the compositional gradient associated with titanium segregation during the growth, the properties vary along the growth axis, which result in property variation from wafer-to-wafer and even within the wafer. Compositional uniformity can be improved by growing along the crystallographic [001] directions, where there is almost no compositional gradient in [001] oriented wafers (radial direction), the axial gradient (along the crystal growth direction), however, possess yet high composition segregation. Three different regions can be identified in the as-grown [001] seeded PMNPT crystal boule by observing the transparency and spontaneous domain structure in the polished wafers, sliced along the growth direction. In the lower part of the boule, where crystallization occurs first, the crystal is semi-transparent and uniform without visible ferroelectric macrodomains, corresponding to the rhombohedral phase. In the middle part, the crystal is very cloudy due to light scattering by large and irregular-shaped visible domains, relate to the monoclinic phase. In the upper part, however, the crystal becomes transparent with only $90^{\circ}$ domains observed, indicative of the tetragonal phase. ${ }^{186}$

Figure 7 shows a typical composition profile of Ti concentration in a [001]-oriented crystal boule grown by the Bridgman method. The Ti concentration was found to be about 0.27 at the initial part, slightly increasing to 0.36 at the top of the crystal boule. The compositional profile of Ti was simulated by a well-established equation used for describing the compositional segregation behavior in solid solution systems during the normal solidification process

$$
C_{s}=k_{e} C_{0}(1-g)^{k_{e}-1}
$$

where $k_{\mathrm{e}}$ is effective segregation coefficient, $C_{\mathrm{s}}$ and $C_{0}$ are the solute concentration in the crystal and the starting melt, respectively, and $g$ is the solidified fraction. The effective segregation coefficient was found to be about $\sim 0.83$. Thus, Ti segregation in PMN-PT gives rise to the compositional inhomogeneity and accounts for the property variation, resulting in only one third of the as-grown crystal boule with desired dielectric and piezoelectric properties. ${ }^{186}$ Owing to the inevitable compositional segregation in the Bridgman growth, alternative crystal growth techniques have to be sought to further improve the longitudinal compositional

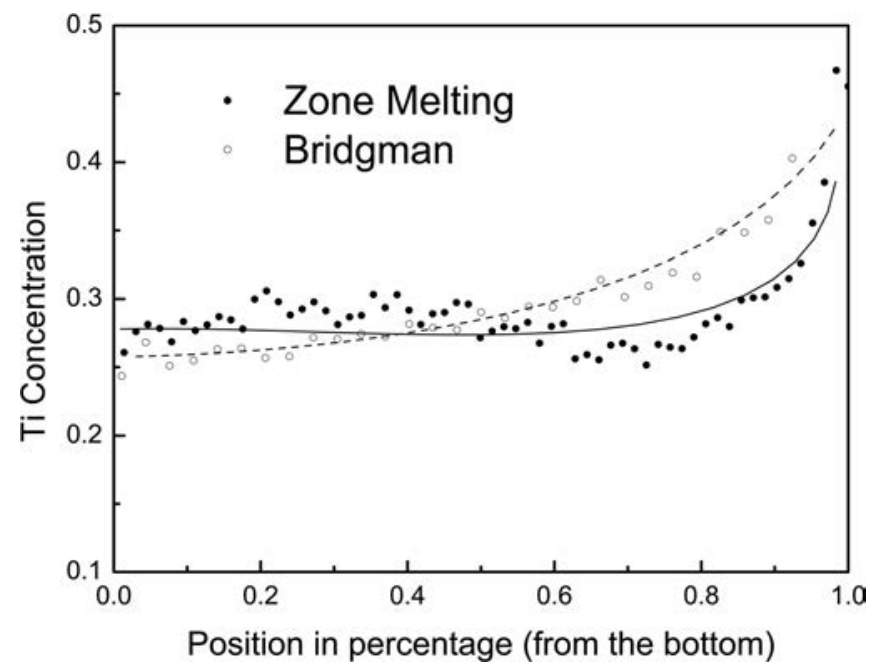

FIG. 7. Ti concentration along the growth direction in PMN-PT crystal boules grown by Bridgman and Zone-melting methods (data from Ref. 186). 
uniformity along the growth direction and to extend the portion of as-grown boules with optimal piezoelectric properties. Zone leveling, which has been shown to produce a uniform distribution of solute in single crystals, has been proposed as an alternative method to prevent compositional segregation. ${ }^{181,182,185,186}$ In single-pass zone leveling, the composition distribution along the solidification direction can be described by the following equation:

$$
C_{s}=C_{0}\left(1-\left(1-k_{e}\right) e^{-k_{e} d / l}\right)
$$

where $d$ is the distance from the point at which the first solid freezes and $l$ is the length of the melting zone. Theoretically, zone leveling can produce a flat compositional distribution except for the initial and ending regions. The zone melting growth of PMN-PT crystals was initially demonstrated using a $r f$ induction heated furnace by self-seeding growth. ${ }^{181}$ Recently, [110]-oriented PMN-PT single crystals have also been grown by a modified zone melting process in a threezone resistance heating furnace. ${ }^{186}$ The Ti concentration along the growth direction in this boule is given in Figure 7 and compared with the conventional Bridgman method. It is obvious from the data that the zone melting method offers improved longitudinal compositional uniformity, by leveling the Ti concentration in the middle part of the boule.

For PMN-PT and PIN-PMN-PT crystal growth, two major modifications to the conventional Bridgman method have been adopted: multi-crucible configuration and zone leveling, leading to the cost-effective manufacturing of large crystals. ${ }^{185,186}$ In order to further reduce crystal cost, it is important to improve the compositional segregation. Continuous top-feeding Bridgman method, which maintains steady state for melt composition and the mass transport rate, has been explored in last few years. Several companies, including JFE Mineral Co., ${ }^{201}$ TRS Technologies, ${ }^{202}$ and HC Materials, ${ }^{203}$ have initiated this modification, with preliminary results showing that two thirds of the as-grown crystals possess uniform dielectric and piezoelectric properties, greatly increasing the homogeneity and yield, thus decrease the overall production cost. ${ }^{201}$

\section{Solid state conversion}

The solid state conversion method (SSCG or template grain growth (TGG) $)^{7,204-216}$ has been studied as an alternative approach to achieve single crystals. In the SSCG process, a small single crystal seed is diffusion-bonded to a highly dense polycrystalline body. Growth occurs by consuming fine grains in the matrix to become a large single crystal after long annealing period, generally at temperatures $100-200{ }^{\circ} \mathrm{C}$ below melting points. This process is referred to as solid-state single crystal growth because it does not involve complete melting of the major components. Thus, the SSCG method is useful for the single crystal growth of materials with high melting temperatures, volatile components, and incongruent melting. ${ }^{216,217}$ Compared to the other single crystal growth processes, the crystal growth rate in the SSCG process is relatively low, being in the range of $50 \mu \mathrm{m}$ to $200 \mu \mathrm{m}$ per $\mathrm{h}$, depending on the material system. ${ }^{216}$
For SSCG of PMN-PT, excess PbO (up to $\sim 8$ wt. $\%$ ) is generally added to the compound powder and mixed thoroughly, then pressed into pellets and sintered to obtain high density ceramics. Abnormal grain growth (AGG) or exaggerated grain growth is observed to occur with the formation of a small amount of liquid during annealing at $1200^{\circ} \mathrm{C}$ for several hours. During AGG, a few grains grow much faster than the surrounding fine grain matrix so that the microstructure exhibits a bimodal distribution of grain size. ${ }^{216}$ Abnormal grains usually grow until they impinge upon each other. If it is possible to control the nucleation of abnormal grains in a polycrystalline material, AGG can be used to grow a single crystal. In the SSCG process, a small [011] oriented $\mathrm{Ba}\left(\mathrm{Zr}_{0.1-}\right.$ $\left.\mathrm{Ti}_{0.9}\right) \mathrm{O}_{3}(\mathrm{BZT})$ single crystal seed is embedded or bonded on the surface of the polycrystalline ceramic. In spite of the difference in chemical composition between the seed crystals and the ceramic, the BZT seed crystals are chemically stable in the Pb-based ceramics and thus act as an effective seed single crystal and PMN-PT crystals can be obtained. ${ }^{216}$

In order to increase Curie temperature, PMN-PT modified by $\mathrm{PbZrO}_{3}$ (PZ) end member was attempted using flux and Bridgman methods, with limited success due to the incongruent melting feature of PZ. During the growth, PZ easily decomposes to $\mathrm{ZrO}_{2}$, preventing the continuous growth of PZT or PMN-PZT single crystals. From this perspective, the SSCG method is potential to grow crystals with PZ end member, where no melting of the compound is involved. The as grown PMN-PZT single crystal with size up to $60 \mathrm{~mm}$ was reported to be successfully fabricated by the SSCG approach, ${ }^{216,217}$ showing high Curie temperature and ferroelectric phase transition temperatures, being on the order of $>200^{\circ} \mathrm{C}$ and $96-170^{\circ} \mathrm{C}$, respectively, with comparable dielectric, piezoelectric, and electromechanical properties to PMN-PT crystals. ${ }^{127-130}$

\section{STRUCTURE AND PROPERTY CHARACTERIZATIONS}

\section{A. Crystal phase determination}

As introduced in Sec. II B, due to the nature of Bridgman growth method, the composition may not be uniform along the crystal boule, with rhombohedral (R), orthorhombic/monoclinic $(\mathrm{O})$, and tetragonal $(\mathrm{T})$ phases possible. ${ }^{186}$ Furthermore, for relaxor-PT crystals with a composition close to an MPB, the phase may vary by poling or annealing treatment. Therefore, it is important to determine the specific phase of the crystal to be characterized. In the following, the general phase characterization methods will be discussed, with emphasis on macroscopic methods.

\section{Microscopic characterization}

Microscopic characterization of crystals includes X-ray diffraction (XRD) and PLM, which can be used to directly determine the phase.

The lattice parameters and phase of the materials can be readily determined from XRD analysis. However, for relaxorPT crystals with a composition close to an MPB, the lattice parameters of $\mathrm{R}, \mathrm{O}$, and $\mathrm{T}$ phases are similar and difficult to 
distinguish. Therefore, high energy XRD methods have been applied to characterize the phase of relaxor-PT crystals. $^{218}$ Alternatively, the phases can be identified according to extinction angles using PLM method. ${ }^{219}$ The advantage of this technique is that it can be easily used to observe the variation of crystal phase as a function of electric field, stress, or temperature. $^{220-224}$

\section{Macroscopic characterization}

The phase of crystals can also be determined indirectly, using piezoelectric, dielectric permittivity-vs-temperature $(\varepsilon-\mathrm{T})$, and strain-vs-electric field (S-E) measurements. ${ }^{116,225,226}$ In the following sections, the phase characterization methods are introduced for relaxor-PT crystals with various poling directions.

a. [111] poled relaxor-PT crystals. For [111] poled crystals, it is easy to distinguish the $\mathrm{R}$ phase from $\mathrm{T}$ or $\mathrm{O}$ phase, by direct $d_{33}$ measurements, owing to the fact that very low $d_{33}$ values can be observed for [111] poled R crystals $(<200 \mathrm{pC} / \mathrm{N})$. Low $d_{33}$ values can be explained as follows. In the $\mathrm{R}$ phase, the spontaneous polarization lies along the $\langle 111\rangle$ crystallographic direction, with a single domain state "1 R" obtained when poled along [111] directions, where no polarization rotation contributes to the longitudinal $d_{33}$. The same phenomena can be observed in [011] poled orthorhombic crystals and [001] poled tetragonal crystals, with single domain states of " 10 " and " $1 \mathrm{~T}$," respectively. To distinguish [111] poled $\mathrm{O}$ and $\mathrm{T}$ crystals, the $\varepsilon$ - $\mathrm{T}$ measurements can be used, since an O-T phase transition can be observed for $\mathrm{O}$ crystals prior to the Curie temperature, while no phase transitions exist for $\mathrm{T}$ crystals above room temperature, until reaching the Curie temperature.

b. [011] poled relaxor-PT crystals. Analogous to [111] poled crystals, $d_{33}$ measurements can also be used to distinguish [011] poled O crystals from R and T crystals, the [011] poled $\mathrm{R}$ and $\mathrm{T}$ crystals can be distinguished by $\varepsilon$ - $\mathrm{T}$ measurements, since no ferroelectric phase transition occurs for $\mathrm{T}$ crystals above room temperature.

c. [001] poled relaxor-PT crystals. Similar to the case of [111] and [011] poled crystals, the [001] poled T crystals can be readily determined by $d_{33}$ or $\varepsilon$-T measurements. However, distinguishing $\mathrm{R}$ and $\mathrm{O}$ phases is complicated for [001] poled crystals, because $d_{33}$ values are similar for [001] poled $\mathrm{R}$ and O crystals, with " $4 \mathrm{R}$ " and " $4 \mathrm{O}$ " engineered domain configurations, respectively. By systematically studying of $\varepsilon$-T and $\mathrm{S}-\mathrm{E}$ behavior, the methods to distinguish $\mathrm{R}$ and $\mathrm{O}$ phases for relaxor-PT crystals were proposed, ${ }^{116,225}$ where the phase could be determined according to the temperature or electric field induced phase transitions and field induced strain level.

\section{B. Dielectric and piezoelectric measurements}

The dielectric permittivity of relaxor-PT crystals can be determined by measuring the capacitance of plate samples with electrodes on the major surfaces. To measure the "free" dielectric permittivity $\varepsilon_{i j}{ }^{T}$, the test frequency should be sub- stantially lower than the lowest resonance frequency (generally at $1 \mathrm{kHz}$ ). The "clamped" dielectric permittivity $\varepsilon_{i j} S$ can be determined at a frequency which is high compared to the principal natural frequencies of the plate samples (usually two times the principal thickness antiresonance frequency). Compared to $\varepsilon_{i j}^{S}$ parameters, electromechanical coupling $k_{i j}$ can be measured with improved accuracy, ${ }^{227}$ therefore, $\varepsilon_{i j} S$ is usually determined by the following equation:

$$
\varepsilon_{33}^{S} / \varepsilon_{33}^{T}=1-k_{33}^{2}
$$

Due to the anisotropy of relaxor-PT crystals, only three dielectric permittivities $\varepsilon_{33}, \varepsilon_{22}$, and $\varepsilon_{11}$ are non-zero parameters exist in the dielectric matrix. The permittivity $\varepsilon_{33}$ may be determined along the poling direction, while $\varepsilon_{11}$ and $\varepsilon_{22}$ are determined along the directions perpendicular to poling direction. Thus, in order to obtain $\varepsilon_{11}$ or $\varepsilon_{22}$, the electrodes should be removed after the poling process and subsequently re-electroded on the large faces vertical to the original electrode planes.

In contrast to the determination of dielectric permittivities, there are three common measurements that can be used to determine piezoelectric coefficients, i.e., Berlincourt $d_{33}$ meter (direct method), the slope of strain-electric field (converse method) and impedance/ultrasonic pulse-echo method. Other approaches, such as laser interferometer, laser scanning vibrometer or laser velocimeter, have been reported to measure strain of thin film or $d_{33}$ under hard drive conditions, which can be found in Refs. 137, 228, and 229. The Berlincourt and strain-electric field methods are quasi-static, where the testing frequency should be much lower than the fundamental resonance frequency of the sample, being generally used to determine the piezoelectric coefficients $d_{33}$, $d_{31}$, and $d_{32}$ for relaxor-PT crystals.

Compared with the quasi-static method, the impedance and ultrasonic pulse-echo methods are based on the theory of acoustic vibration, which can be used to determine various piezoelectric coefficients and elastic constants, including shear parameters. For the impedance method, the elastic constants and electromechanical coupling factors are calculated by measuring resonance and antiresonance frequencies of the piezoelectric elements, from which, the piezoelectric coefficients can be derived. In order to obtain a certain vibration mode, the sample geometry must meet required aspect ratio. ${ }^{227}$ It should be noted that the general impedance method is not available for lossy materials, whose piezoelectric coefficients can be determined by different approaches, ${ }^{230-234}$ such as impedance spectrum fitting. ${ }^{232,233}$ The ultrasonic pulse-echo measurement can be applied to determine the elastic stiffness constants from the phase velocities of ultrasonic waves propagating along appropriate pure mode directions. By using the impedance and ultrasonic methods, the full matrix of dielectric/elastic/piezoelectric constants have been determined for relaxor-PT crystals with various phases (rhombohedral, orthorhombic, and tetragonal). $31,71,72,97,112,115,122,235,236$

\section{Loss determination}

For piezoelectric materials, three different losses may be considered: dielectric, mechanical, and piezoelectric losses. ${ }^{237}$ The loss factors can be represented as follows: 


$$
\begin{aligned}
& \varepsilon=\varepsilon^{\prime}\left(1-j \tan \delta_{E}\right) \\
& s=s^{\prime}\left(1-j \tan \delta_{M}\right) \\
& d=d^{\prime}\left(1-j \tan \delta_{P}\right)
\end{aligned}
$$

where $\tan \delta_{\mathrm{E}}, \tan \delta_{\mathrm{M}}$, and $\tan \delta_{\mathrm{P}}$ are dielectric, mechanical, and piezoelectric loss factors, respectively. To determine the loss factors, hysteresis loop and inductance-capacitanceresistance (LCR) meter measurements can be utilized.

\section{Hysteresis loop measurements}

The losses are associated with the hysteresis phenomena in materials. The determination of loss factors is introduced in the Ref. 36. A typical $D-E$ hysteresis loop is shown in Figure 8, with applied electric field $E_{0} \cos \omega t$. It can be observed that a larger loss factor $\tan \delta_{E}$ corresponds to a higher level of $D-E$ hysteresis. From Figure 8, the stored electric energy $U_{E}$ (E from 0 to $E_{0}$ ) and the loss energy $\left(w_{\mathrm{E}}\right)$ of an electric field cycle (area $A$ ) can be represented as follows:

$$
\begin{gathered}
U_{E}=\frac{1}{2} \varepsilon^{\prime} E_{0}^{2} \\
A=w_{E}=\pi \varepsilon^{\prime} E_{0}^{2} \tan \delta_{E}
\end{gathered}
$$

where loss factors are considered to be independent of electric field. From the equations, the dielectric loss factor can be calculated

$$
\tan \delta_{E}=\frac{w_{E}}{2 \pi U_{E}}
$$

Thus, the dielectric loss can be determined by measuring the area $A$, as shown in Figure 8. Based on the same method, the mechanical and piezoelectric losses can be determined by measuring strain-stress and strain-electric field loops, respectively.

\section{Impedance spectrum measurements}

The dielectric loss can be directly measured using an $L C R$ meter, while the mechanical loss factors can be determined by measuring the frequencies at resonance and $-3 \mathrm{~dB}$ bandwidth, as discussed in Sec. I B $1 \mathrm{f}$. However, it is hard to determine piezoelectric loss by measuring the impedance

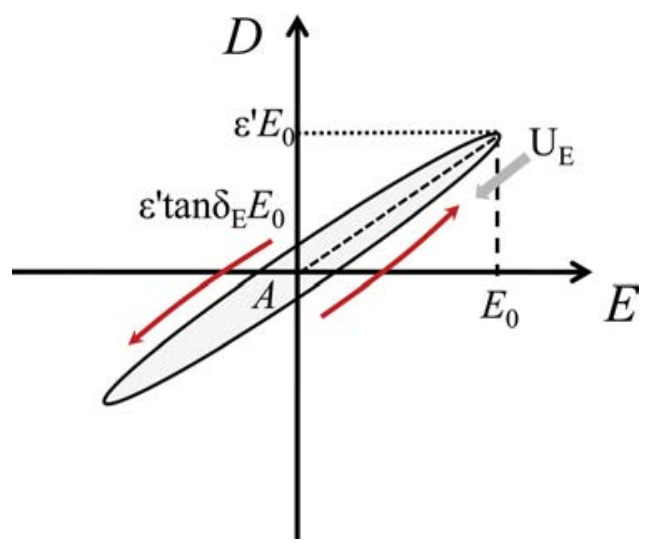

FIG. 8. (Color online) Schematic plot for D-E loop with certain dielectric loss $\tan \delta_{\mathrm{E}}$. spectrum, as discussed in Ref. 238, resulting in inconsistent results. ${ }^{232,238,239}$

\section{Determination of full matrix material constants}

In general, domain engineered Relaxor-PT single crystals possess extraordinary large piezoelectric coefficients for the longitudinal vibration mode, while crystals with single domain states are found to possess very low longitudinal properties and ultrahigh shear properties. Thus, it is desirable to understand the fundamental principle of domain engineering and to understand the physical origin of the ultrahigh piezoelectric and electromechanical properties, which requires the full set of material constants for theoretical analysis. From application viewpoint, in order to use computer simulations (such as the ATILA finite element package) to design transducer devices, the complete set of material constants must be obtained for input in the software.

There are several issues involved in the characterization of the high performance relaxor-PT crystals, which should be taken care of during the determination of the full matrix material constants. ${ }^{31}$ For single domain crystals, the samples are poled along their respective spontaneous polarization directions. However, a single domain state may not be stable and domains may partially switch back upon removal of the poling field, leading to inaccurate results. To overcome this, a $d c$ bias field may be used to stabilize the single domain state, at a price of inducing the nonlinear behavior. For ternary PIN-PMN-PT crystals, the single domain states were found to be stable, allowing the determination of full matrix material constants. ${ }^{115}$

For samples with domain engineered configurations, such as "4R," they are in multi-domain state, whose macroscopic symmetry is the effective symmetry of the engineered domain patterns, which can be very different from the microscopic crystal symmetry. Because several energetically degenerated domain states exist in a given structure and several domain wall orientations are allowed, the engineered domain patterns can be complicated and the symmetries of such patterns need to be analyzed for different combinations of domains and domain walls. ${ }^{31}$

For [001] poled relaxor-PT crystals, including the domain engineered structures " $4 \mathrm{R}$," "4O," and single domain " $1 \mathrm{~T}$," the macroscopic symmetry is $4 \mathrm{~mm}$ (as shown in Table I), where the $\mathrm{Z}$ axis is along the poling direction [001], with $\mathrm{X}$ and $\mathrm{Y}$ axes along [100] and [010] crystallographic directions to form a right-hand orthogonal coordinate. There are eleven independent electroelastic constants in $4 \mathrm{~mm}$ symmetry. For [011] poled $\mathrm{R}$ and $\mathrm{O}$ crystals, including the domain engineered structure " $2 \mathrm{R}$ " and single domain state " 10 ," the macroscopic symmetry is $\mathrm{mm} 2$, with seventeen independent electroelastic constants. The $\mathrm{Z}$ axis is along the poling direction [011], with $\mathrm{X}$ and $\mathrm{Y}$ axes along the crystallographic directions [011] and [100], respectively. For [111] poled R crystals, the single domain state " $1 \mathrm{R}$ " has a macroscopic symmetry $3 \mathrm{~m}$, with twelve independent electroelastic constants. The $\mathrm{Z}$ axis is along poling direction [111], while $\mathrm{X}$ and $\mathrm{Y}$ axes are along [110] and [11 $\overline{2}$ ] directions, respectively. In order to obtain the full matrix material constants, various crystal samples with different geometries and orientations need to be prepared according to symmetry constraints and constitutive relations, as given in Figure 9, 

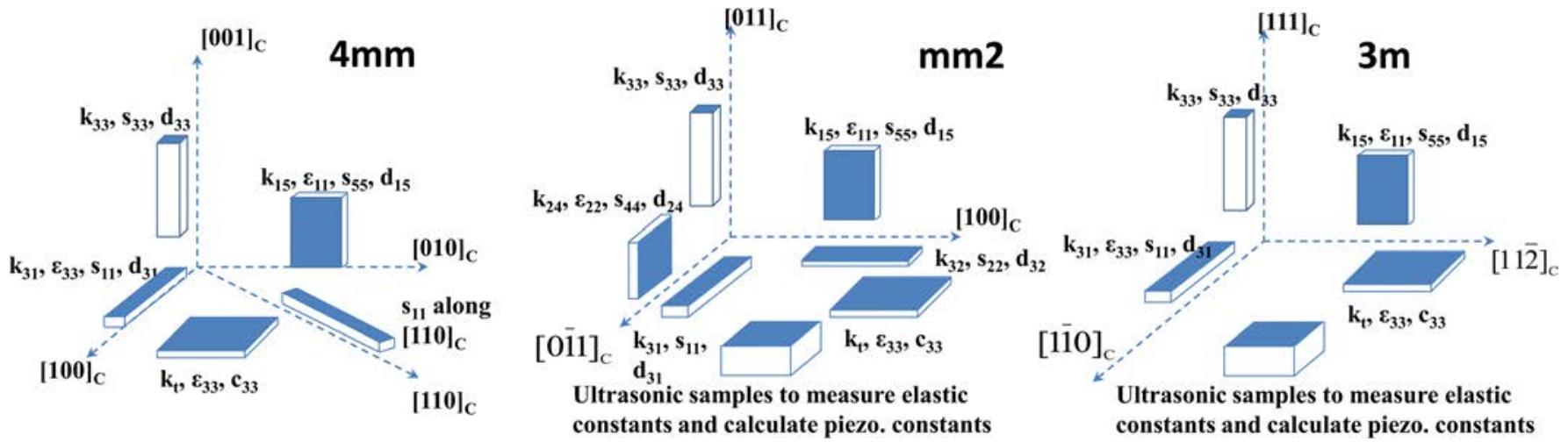

FIG. 9. (Color online) Samples for the full matrix material constants determination, for crystals with $4 \mathrm{~mm}$, mm2, and $3 \mathrm{~m}$ symmetries.

according to Institute of Electrical and Electronics Engineers (IEEE) standard on piezoelectricity, ${ }^{227}$ the dielectric, elastic, piezoelectric, and electromechanical constants can be calculated and/or derived. ${ }^{97,240,241}$

Another critical issue for the full matrix determination is self-consistency of the obtained data, which may be induced by experimental measurement, uncertainties of some derived quantities due to the unstable nature of some constitutive relations, which will enlarge the error through error propagation. ${ }^{31,242}$ For relaxor-PT crystals, the lack of compositional uniformity will lead to large property variation. Thus, it is desirable to reduce the number of samples utilized in the full matrix determination, in order to eliminate the inhomogeneous compositional effect. It has been reported that the combination of the impedance and ultrasonic pulse-echo methods offer the advantage of using less samples to determine material constants, where the different phase velocities (relate to elastic stiffness constants) can be measured in one cubic sample. . $^{31,71,72,115,226,243-245}$ Table III lists the phase velocities and related elastic stiffness constants for $4 \mathrm{~mm}$ ([001] poled crystals), $\mathrm{mm} 2$ ([011] poled crystals), and $3 \mathrm{~m}$ ([111] poled crystals) symmetries, based on the measured shear and longitudinal waves with different propagation and displacement directions, from which, most of the elastic constants can be obtained. ${ }^{71,115,243}$

Table IV lists some piezoelectric and dielectric constants for single domain relaxor-PT crystals. As shown in Table IV, a critical characteristic of relaxor-PT crystals is that the longitudinal properties $\left(\varepsilon_{33}, d_{33}\right.$, and $\left.\mathrm{s}_{33}\right)$ are much smaller than those values of shear vibration $\left(\varepsilon_{11}, d_{15}\right.$, and $\left.s_{55}\right)$. For single domain crystals, the longitudinal piezoelectric response is mainly related to polarization extension, while the shear piezoelectric response is associated with polarization rotation, as analyzed by Damjanovic et al. ${ }^{246,247}$ The high shear piezoelectric response in relaxor-PT crystals is attributed to the ease of polarization rotation, which will be discussed in Sec. IV.

\section{E. Piezoelectric properties as function of orientation and composition}

\section{Orientation dependent properties}

Table $\mathrm{V}$ lists the longitudinal properties of various relaxor-PT crystals as a function of crystallographic orientation. The longitudinal piezoelectric, dielectric, and elastic constants of the crystals poled along nonpolar directions ( $\mathrm{R}$ crystals along [001] and [011] directions and $\mathrm{O}$ crystals along [001] direction) are found to be much higher than those coefficients along the polar direction ( $\mathrm{R}$ crystals along [111] and $\mathrm{O}$ crystals along [011]). Figure 10 shows the domain configuration of [001] poled R and O crystals. In " $4 \mathrm{R}$ " and " $4 \mathrm{O}$ " domain configurations, the domain vectors lying along different directions are equivalent to the [001] orientation. Therefore, without considering the contribution of domain walls, the

TABLE III. Relationship between the phase velocity and elastic constants for crystals with $4 \mathrm{~mm}$, mm2, and $3 \mathrm{~m}$ symmetries in the pulse-echo ultrasonic measurements.

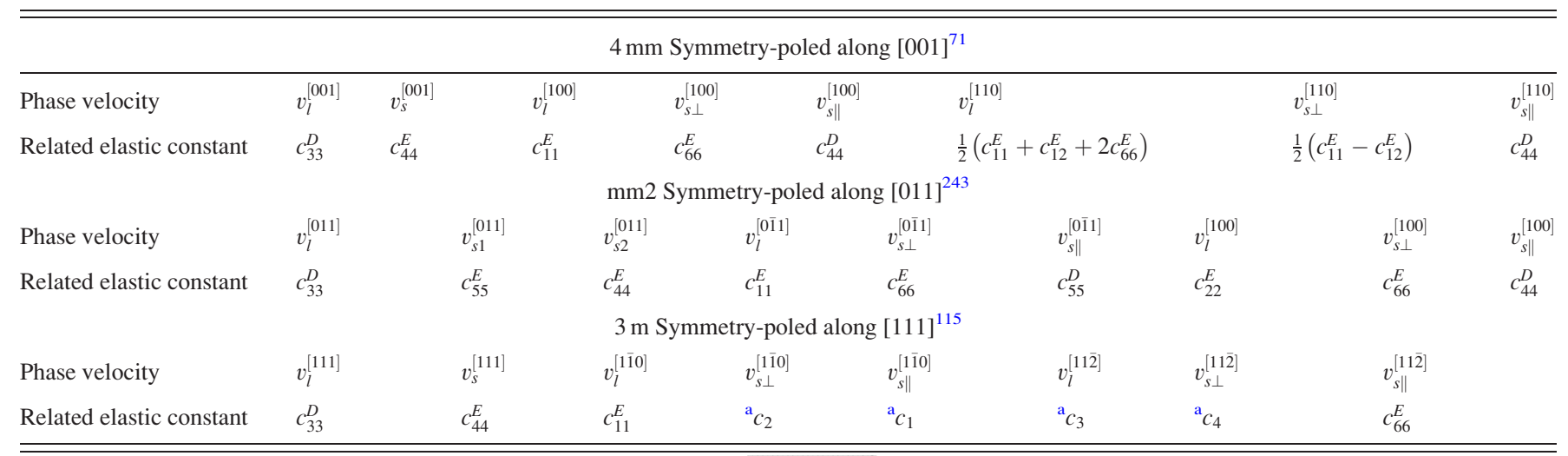

${ }^{\mathrm{a}}$ For $3 \mathrm{~m}$ symmetry, $c_{44}^{D}=\frac{c_{1} c_{2}-c_{3} c_{4}}{c_{1}+c_{2}-c_{3}-c_{4}} ; c_{66}^{D}=c_{1}+c_{2}-c_{44}^{D} ; c_{14}^{D}=\sqrt{c_{44}^{D} c_{66}^{D}-c_{1} c_{2}}$. 
TABLE IV. Piezoelectric, dielectric, and elastic constants for single domain PIN-PMN-PT crystals with various phases. ${ }^{\text {a }}$

\begin{tabular}{|c|c|c|c|c|c|c|c|c|c|c|c|c|c|c|c|c|}
\hline \multirow[b]{2}{*}{ Crystal phase } & \multicolumn{5}{|c|}{ Dielectric constants } & \multicolumn{11}{|c|}{ Elastic constants $\mathrm{s}_{\mathrm{ij}}\left(\times 10^{-12} \mathrm{~m}^{2} / \mathrm{N}\right)$} \\
\hline & $\varepsilon_{33}^{T} / \varepsilon_{0}$ & \multicolumn{2}{|r|}{$\varepsilon_{22}^{T} / \varepsilon_{0}$} & \multicolumn{2}{|l|}{$\varepsilon_{11}^{T} / \varepsilon_{0}$} & \multicolumn{2}{|l|}{$\mathrm{s}_{11}{ }^{\mathrm{E}}$} & $\mathrm{s}_{22}^{\mathrm{E}}$ & \multicolumn{2}{|c|}{$\mathrm{s}_{33}{ }^{\mathrm{E}}$} & \multicolumn{2}{|l|}{$\mathrm{s}_{44}{ }^{\mathrm{E}}$} & \multicolumn{2}{|l|}{$\mathrm{s}_{55}{ }^{\mathrm{E}}$} & \multicolumn{2}{|c|}{$\mathrm{s}_{66}{ }^{\mathrm{E}}$} \\
\hline Tetragonal $1 \mathrm{~T}^{112}$ & 1090 & & 15000 & 15000 & & 17.1 & & 17.1 & 41.0 & & 55.0 & & 55.0 & & 25.0 & \\
\hline Orthorhombic $1 \mathrm{O}^{243}$ & 1500 & & 30000 & 8070 & & 9.2 & & 21.2 & 16.8 & & 78.1 & & 316 & & 15.5 & \\
\hline Rhombohedral $1 \mathrm{R}^{115}$ & 700 & & 6300 & 6300 & & 10.4 & & 10.4 & 6.8 & & 101.9 & & 101.9 & & 33.8 & \\
\hline \multirow[t]{2}{*}{ Crystal phase } & \multicolumn{9}{|c|}{ Piezoelectric constants $\mathrm{d}_{\mathrm{ij}}(\mathrm{pC} / \mathrm{N})$} & \multicolumn{7}{|c|}{ Electromechanical coupling factors } \\
\hline & $d_{31}$ & $d_{32}$ & & & $d_{15}$ & $d_{24}$ & $d_{22}$ & $d_{16}$ & $k_{31}$ & $k_{32}$ & & $k_{33}$ & & $k_{15}$ & & $k_{24}$ \\
\hline Tetragonal $1 \mathrm{~T}^{112}$ & -200 & -200 & & & 2350 & 2350 & 0 & 0 & 0.50 & 0.50 & & 0.84 & & 0.85 & & 0.85 \\
\hline Orthorhombic $10^{243}$ & 153 & -346 & & & 4550 & 4100 & 0 & 0 & 0.44 & 0.65 & & 0.74 & & 0.96 & & 0.90 \\
\hline Rhombohedral $1 \mathrm{R}^{115}$ & -34 & -34 & & & 2190 & 2190 & 511 & -1022 & 0.13 & 0.13 & & 0.36 & & 0.92 & & 0.92 \\
\hline
\end{tabular}

${ }^{\mathrm{a}}$ The properties are expected to increase with composition approaching an MPB.

contribution of domains to longitudinal piezoelectricity can be analyzed from single domain properties. Figure 11 shows the orientation dependence of piezoelectric coefficients $d_{33}^{*}$ for PIN-PMN-PT crystals, calculated from the data in single domain states "1 R," "1 T," and "1O," respectively, where the maximum longitudinal piezoelectric responses are along their nonpolar directions. A high anisotropic $d_{33}^{*}$ is observed for all single domain crystals, which can be attributed to the high piezoelectric anisotropic factor $d_{15} / d_{33}(>4)$. It was reported that the calculated $d_{33}^{*}$ along [001] direction (derived from single domain data) was about $80 \% \sim 90 \%$ of measured $d_{33}{ }^{*}$ for [001] poled domain engineered crystals, ${ }^{248,249}$ demonstrating that the irreversible (extrinsic) contribution to piezoelectric coefficient is relatively small. According to Rayleigh analysis, ${ }^{225}$ the irreversible piezoelectric contribution was about $10 \%-20 \%$ for crystals with MPB compositions, while it was only about $\sim 3 \%$ for relaxor-PT crystals with compositions far away from an MPB. It was also confirmed in [001] poled domain engineered PIN-PMN-PT crystals with O phase, where the calculated $d_{33}^{*}$ value from " 10 " single domain data was found to be about $\sim 11 \%$ lower than the value measured directly, ${ }^{243}$ exhibiting the extrinsic contribution being on the order of $\sim 11 \%$, due to the irreversible electric-fieldinduced-phase transition. ${ }^{116,225}$ Thus, it can be concluded that the major contribution of the ultrahigh piezoelectric $d_{33}^{*}$ in domain engineered crystals comes from the orientation conver- sion of the high shear piezoelectric $d_{15}$ in the single domain state. The origin of ultrahigh longitudinal piezoelectric response of relaxor-PT crystals will be discussed in detail in Sec. IV A.

The dielectric loss and mechanical quality factor also exhibit anisotropic characteristics. Table VI lists the dielectric loss and mechanical quality factors for PMN-PT crystals as a function of orientation. ${ }^{250}$ For rhombohedral relaxor-PT crystals, the lowest loss factor was found to be along their respective polar directions, with mechanical $Q_{m}$ values being $>1000$. Of particular significance is that both high electromechanical coupling $(\sim 0.9)$ and large mechanical $Q_{m}$ ( 600) were achieved in [011] poled crystals with " $2 \mathrm{R}$ " domain engineered configuration, which will be discussed in Sec. IV B.

Table $\mathrm{V}$ lists the shear constants for rhombohedral relaxor-PT crystals poled along [001] and [011]. The shear constants for [111] poled R crystals were listed in Table IV. In contrast to the longitudinal piezoelectric response, the shear piezoelectric response of domain engineered crystals cannot be simply analyzed by anisotropic calculations. Figure 12 shows the orientation dependence of the shear piezoelectric coefficient $d_{15}^{*}$ for single domain rhombohedral crystals. For " $1 \mathrm{R}$ " single domain state, the shear coefficient $d_{15}^{*}$ is $\sim 4600 \mathrm{pC} / \mathrm{N}$ after rotation around $[1 \overline{1} 0]$ ( $x$ axis) by $54^{\circ}$ (new z' axis is along [001] direction), being slightly higher

TABLE V. Comparison of longitudinal and shear piezoelectricity for relaxor-PT crystals. ${ }^{19,31,71,110,114,115,245}$

\begin{tabular}{|c|c|c|c|c|c|c|c|c|c|c|c|c|c|}
\hline \multirow{2}{*}{$\begin{array}{l}\text { Mode } \\
\text { Longitudinal }\end{array}$} & \multirow{2}{*}{$\begin{array}{c}\text { Crystal } \\
\text { Relaxor-PT crystals }\end{array}$} & \multicolumn{4}{|c|}{ [001] poled } & \multicolumn{4}{|c|}{ [011] poled } & \multicolumn{4}{|c|}{ [111] poled } \\
\hline & & $\varepsilon_{33}{ }^{\mathrm{T}} / \varepsilon_{0}$ & $\mathrm{~S}_{33}{ }^{\mathrm{E}} \mathrm{pm}^{2} / \mathrm{N}$ & $\mathrm{d}_{33} \mathrm{pC} / \mathrm{N}$ & $\mathrm{k}_{33}$ & $\varepsilon_{33} \mathrm{~T} / \varepsilon_{0}$ & $\mathrm{~s}_{33}{ }^{\mathrm{E}} \mathrm{pm}^{2} / \mathrm{N}$ & $\mathrm{d}_{33} \mathrm{pC} / \mathrm{N}$ & $\mathrm{k}_{33}$ & $\varepsilon_{33}{ }^{\mathrm{T}} / \varepsilon_{0}$ & $\mathrm{~s}_{33}{ }^{\mathrm{E}} \mathrm{pm}^{2} / \mathrm{N}$ & $\mathrm{d}_{33} \mathrm{pC} / \mathrm{N}$ & $\mathrm{k}_{33}$ \\
\hline & PIN-PMN-PT(R) & 7200 & 77.8 & 2740 & 0.95 & 4400 & 52 & 1300 & 0.92 & 700 & 6.8 & 74 & 0.36 \\
\hline & PMN-PT (R) & 8200 & 120 & 2820 & 0.95 & 3800 & 70 & 1350 & 0.88 & 640 & 13.3 & 190 & 0.69 \\
\hline & PZN-7PT (R) & 5622 & 142 & 2455 & 0.92 & 3180 & 62 & 1150 & 0.87 & - & - & - & - \\
\hline
\end{tabular}

Mode

Material

[001] poled

Shear

\begin{tabular}{ccccc}
\cline { 2 - 5 } Relaxor-PT crystals & $\varepsilon_{11} \mathrm{~T} / \varepsilon_{0}$ & $\mathrm{~s}_{55}{ }^{\mathrm{E}} \mathrm{pm}^{2} / \mathrm{N}$ & $\mathrm{d}_{15} \mathrm{pC} / \mathrm{N}$ & $\mathrm{k}_{15}$ \\
PIN-PMN-PT(R) & 10100 & 14.5 & 230 & 0.20 \\
PMN-PT(R) & 1600 & 14.0 & 146 & 0.32 \\
Ceramics & \multicolumn{4}{c}{ PZT4 } \\
\end{tabular}

Ceramics

\begin{tabular}{|c|c|c|c|c|c|c|c|}
\hline$\varepsilon_{11}{ }^{\mathrm{T}} / \varepsilon_{0}$ & $\mathrm{~s}_{55}{ }^{\mathrm{E}} \mathrm{pm}^{2} / \mathrm{N}$ & $\mathrm{d}_{15} \mathrm{pC} / \mathrm{N}$ & $\mathrm{k}_{15}$ & $\varepsilon_{22}{ }^{\mathrm{T}} / \varepsilon_{0}$ & $\mathrm{~s}_{44}{ }^{\mathrm{E}} \mathrm{pm}^{2} / \mathrm{N}$ & $\mathrm{d}_{24} \mathrm{pC} / \mathrm{N}$ & $\mathrm{k}_{24}$ \\
\hline 6800 & 160 & 2900 & 0.93 & 1480 & 16.1 & 200 & 0.43 \\
\hline 6000 & 140 & 2500 & 0.90 & 1800 & 16.0 & 200 & 0.40 \\
\hline & PZT8 & & & \multicolumn{4}{|c|}{ PZT5H } \\
\hline$\varepsilon_{11}^{\mathrm{T}} / \varepsilon_{0}$ & $\mathrm{~s}_{55}{ }^{\mathrm{E}} \mathrm{pm}^{2} / \mathrm{N}$ & $\mathrm{d}_{15} \mathrm{pC} / \mathrm{N}$ & $\mathrm{k}_{15}$ & $\varepsilon_{11}{ }^{\mathrm{T}} / \varepsilon_{0}$ & $\mathrm{~s}_{55}{ }^{\mathrm{E}} \mathrm{pm}^{2} / \mathrm{N}$ & $\mathrm{d}_{15} \mathrm{pC} / \mathrm{N}$ & $\mathrm{k}_{15}$ \\
\hline 1290 & 31.9 & 330 & 0.55 & 3130 & 43.5 & 740 & 0.68 \\
\hline
\end{tabular}

1290

31.9

330 $\begin{array}{lllll}\varepsilon_{11}{ }^{\mathrm{T}} / \varepsilon_{0} & \mathrm{~s}_{55}{ }^{\mathrm{E}} \mathrm{pm}^{2} / \mathrm{N}_{15} \mathrm{pC} / \mathrm{N} \quad \mathrm{k}_{15}\end{array}$

$\begin{array}{llll}1470 & 39.0 & 496 & 0.71\end{array}$ 
'4R' domain structure

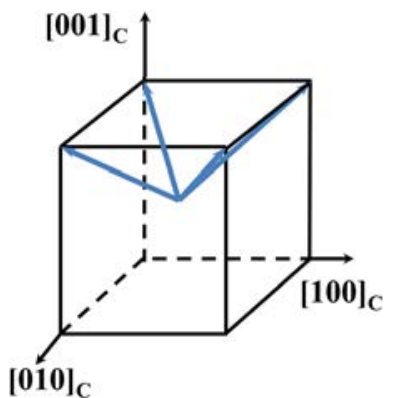

'40' domain structure

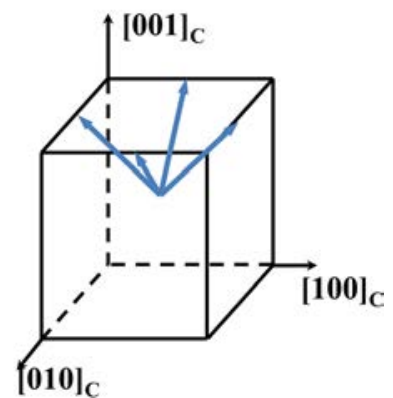

FIG. 10. (Color online) Domain structure (or possible domain vectors) of [001] poled rhombohedral and orthorhombic crystals.

than the original value $(4100 \mathrm{pC} / \mathrm{N}$, without rotation), revealing that $d_{15}$ in [001] poled domain engineered crystals will be on the order of $\sim 4600 \mathrm{pC} / \mathrm{N}$, if only [111] single domain exists, which is not the case. In reality, as listed in Table V, the shear piezoelectric coefficient of [001] poled domain engineered crystals is around $200 \mathrm{pC} / \mathrm{N}$, due to the negating effect among various domains. As shown in Figure 13, with an applying perpendicular electric field along [010], the contribution from [111] and [111] domains to the shear deforma-

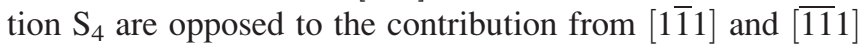
domains, leading to a low level of shear piezoelectricity. Here, it should be noted that $d_{15}=d_{24}$ based on the macroscopic symmetry $4 \mathrm{~mm}$. The negating effect is also observed for the shear coefficient $d_{24}$ in [011] poled " $2 \mathrm{R}$ " crystals, as shown in Figure 14. The contributions of [111] and [1111] to the shear deformation $\mathrm{S}_{4}$ are opposed under an applied field $E_{2}$ along the [100] direction, negating one another and giving rise to the minimized $d_{24}{ }^{235}$ Admittedly, the negating effect does not exist in every shear mode of domain engineered crystals. For the 15 -mode of [011] poled R crystals (" $2 \mathrm{R}$ "
TABLE VI. Mechanical quality factor and dielectric loss of PMN-0.30PT crystals as a function of orientation. ${ }^{250}$

\begin{tabular}{|c|c|c|c|c|c|c|c|c|}
\hline Material & & $\begin{array}{c}\text { Domain } \\
\text { Engineering }\end{array}$ & $\varepsilon_{\mathrm{r}}$ & $\tan \delta$ & $k_{33}$ & $\begin{array}{c}d_{33} \\
(\mathrm{pC} / \mathrm{N})\end{array}$ & $\begin{array}{c}s_{33}{ }^{\mathrm{E}} \\
\left(\mathrm{pm}^{2} / \mathrm{N}\right)\end{array}$ & $\mathrm{Q}_{\mathrm{m}}$ \\
\hline \multirow[t]{3}{*}{ PMN-0.30PT } & [001] & $4 \mathrm{R}$ & 5200 & 0.004 & 0.90 & 1500 & 60.0 & 120 \\
\hline & [011] & $2 \mathrm{R}$ & 4400 & 0.002 & 0.90 & 1050 & 35.9 & 600 \\
\hline & [111] & $1 \mathrm{R}$ & 810 & 0.002 & 0.42 & 90 & 6.5 & 1130 \\
\hline
\end{tabular}

domain configuration), the contribution of the two different domains are identical, both contributing to the shear deformation $S_{5}$ under an applied electric field $E_{1}$ along [0 $\left.\overline{1} 1\right]$, as shown in Figure $14,{ }^{235}$ thus the measured piezoelectric coefficient $\mathrm{d}_{15}(\sim 2500 \mathrm{pC} / \mathrm{N})$ is close to the calculated one $(2100 \mathrm{pC} / \mathrm{N}$, as shown in Figure 12). Based on the results, both orientation dependence of single domain properties (Figure 12) and domain configurations (Figures 13 and 14) should be considered in the analysis of the shear properties of multi-domain crystals.

The orientation dependences of longitudinal and shear piezoelectric responses are discussed according to the coordinate rotation, which are closely related to the domain configurations. It should be noted that by the coordinate rotation, new piezoelectric coefficients can be generated and unwanted coefficients can be eliminated, which are exemplified in the following.

Face (contour) shear piezoelectric coefficient $d_{36}{ }^{*}$ was predicted by coordinate rotation in [011] poled relaxor-PT crystals, according to the equation, ${ }^{251}$

$$
d_{36}^{*}=2 d_{31} \sin \theta \cdot \cos \theta-2 d_{32} \sin \theta \cdot \cos \theta,
$$

where $d_{36}^{*}$ is the face shear coefficient after rotating around the $\mathrm{Z}$ axis by $\theta$ angle and $d_{31}$ and $d_{32}$ are piezoelectric coefficients in the original coordinate of [011] poled R crystals.
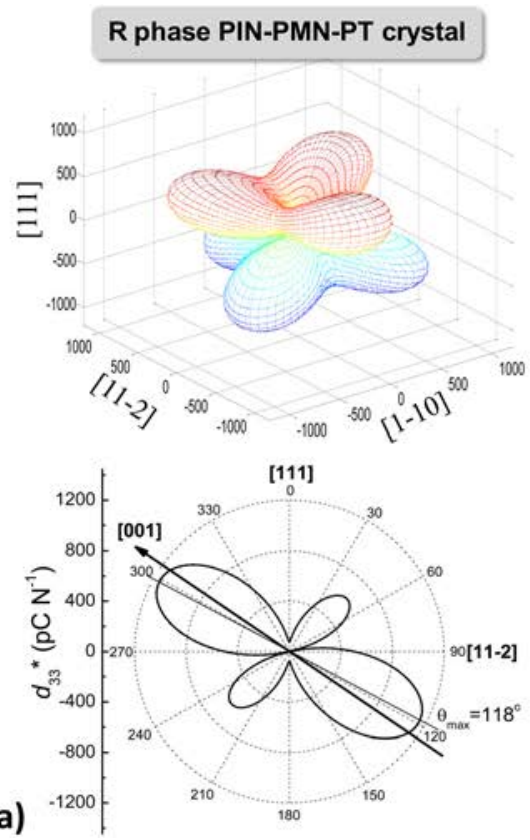
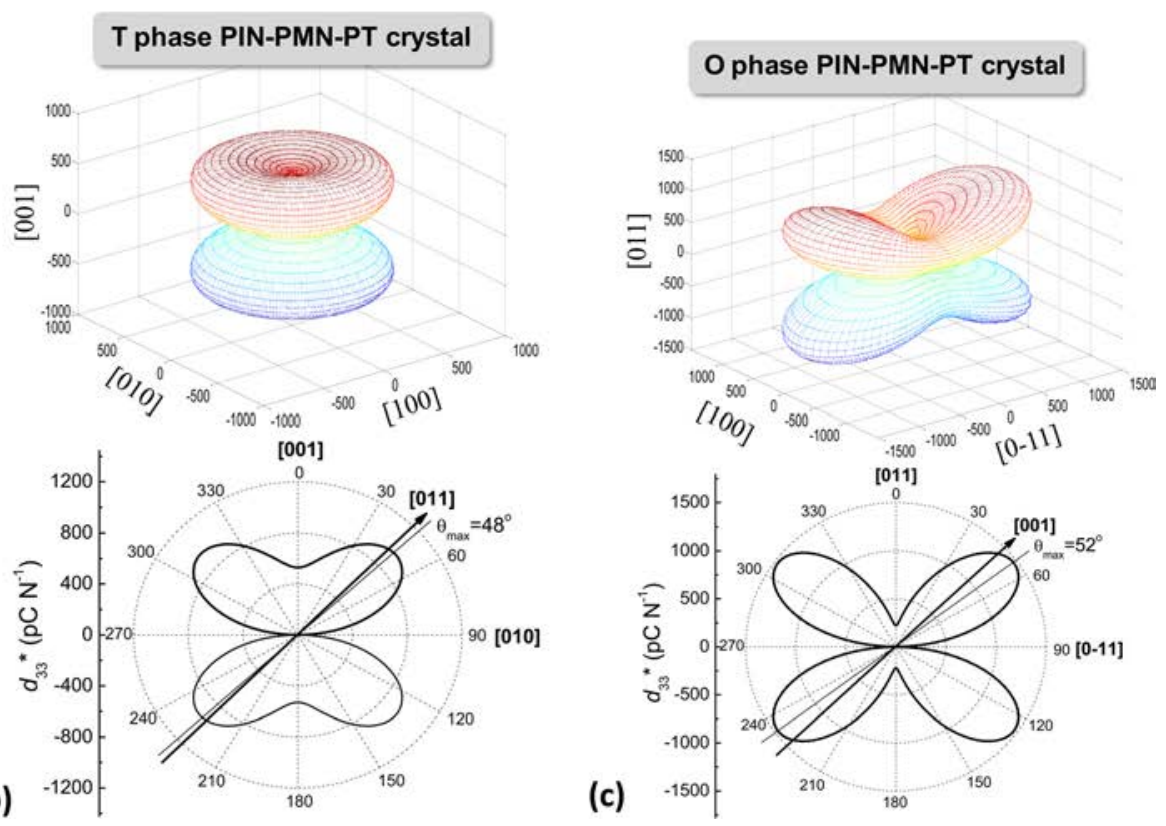

FIG. 11. (Color online) Orientation dependence of piezoelectric coefficient $d_{33}^{*}$ of (a) rhombohedral, (b) tetragonal, and (c) orthorhombic PIN-PMN-PT crystals. Li et al., Advanced Functional Materials 21, 2118, 2011. Copyright $\odot 2011$, Wiley. 


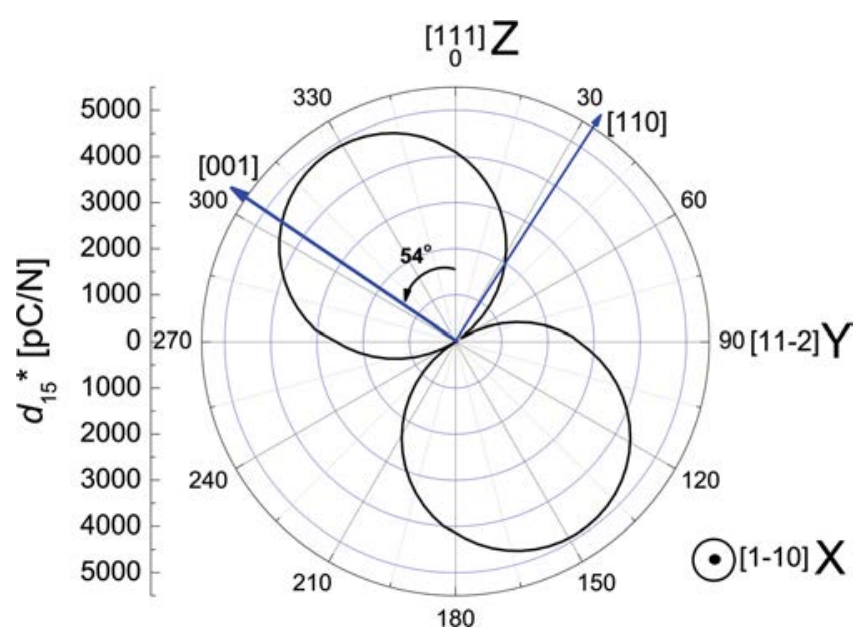

FIG. 12. (Color online) Orientation dependence of shear piezoelectric coefficient for rhombohedral PMN-PT crystals, where input data are from Ref. 72

The maximum face shear $d_{36}^{*}$ was theoretically predicted and experimentally obtained in [011] poled $Z t \pm 45^{\circ}$ relaxor-PT crystals $\left(\theta= \pm 45^{\circ}\right){ }^{251-254}$ The piezoelectric coefficients and electromechanical coupling factors, being on the order of $1600-2800 \mathrm{pC} / \mathrm{N}$ and $0.77-0.83$, respectively, were determined by the impedance method using square plate samples, with ultrahigh elastic compliance constants on the order of $127-225 \mathrm{pm}^{2} / \mathrm{N} .^{254}$ The high elastic compliance corresponds to very low frequency constant, making them good candidates for the low frequency transducers. ${ }^{252-254}$ In addition, compared to $d_{15} / d_{24}$ thickness shear samples, the face shear component can be repolarized since the poling direction is the same as the working electrode direction. The mechanical quality factor of the face shear resonator is $\sim 100-450$, significantly higher than that of thickness shear resonators $(\sim 30){ }^{253,254}$ However, the condition $d_{31}^{*}=d_{32}^{*} \neq 0$ exists in

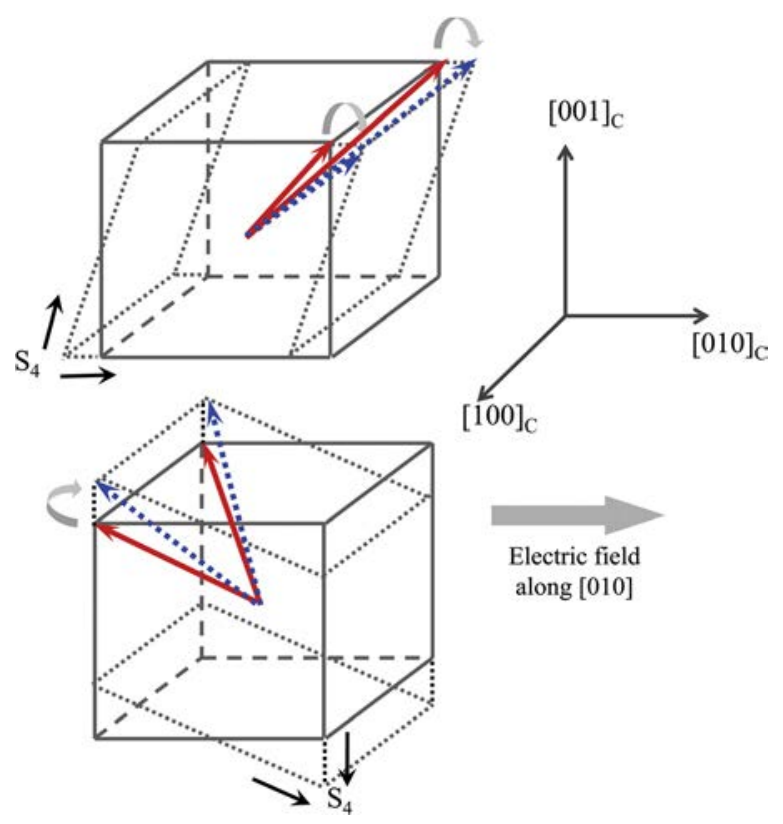

FIG. 13. (Color online) Schematic of polarization rotation for [001] poled $\mathrm{R}$ crystals under [010] perpendicular electric field, related to $d_{24}\left(=d_{15}\right)$.

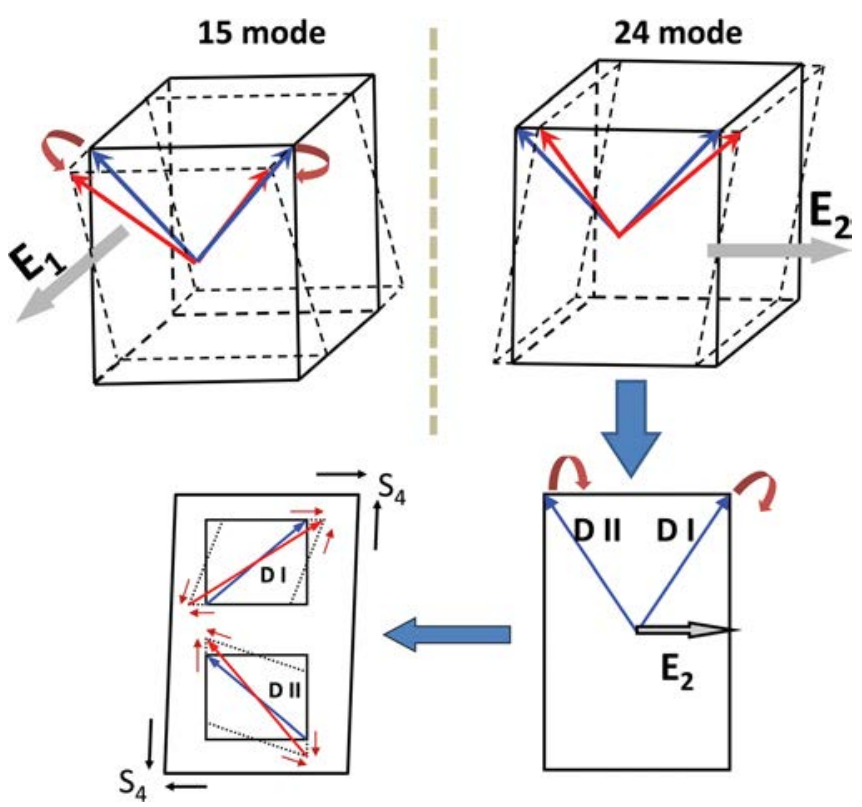

FIG. 14. (Color online) Two independent shear piezoelectric response (15and 24-modes) and related polarization rotation paths in crystals with " $2 \mathrm{R}$ " engineered domain state, where the shear deformations were contributed by polarization rotation of domain I (DI) and domain II (DII). Reprinted with permission from Zhang et al., Applied Physics Letter 97, 132903, 2010. Copyright (C) 2010, the American Institute of Physics.

the newly rotated coordinates, which may interfere with the face shear vibration, thus, it is desired to have zero $d_{31}$ or $d_{32}$ in practical applications. It was reported that one of the transverse extensional piezoelectric coefficients could be eliminated at a rotation angle of

$$
\theta=\arctan \left(\frac{d_{31}}{-d_{32}}\right) \text { or } \theta=\arctan \left(\frac{d_{32}}{-d_{31}}\right),
$$

at the expense of increasing the other transverse extensional coefficient, while the face shear coefficient was found to be insensitive to the rotation angle in the range of $30^{\circ}-60^{\circ} .{ }^{255}$

For rhombohedral crystals with single domain " $1 \mathrm{R}$ " and macroscopic $3 \mathrm{~m}$ symmetries, the thickness shear coefficient $d_{16}$ exists and is equal to $-2 d_{22}$. Analogous to face shear vibration, the thickness shear $d_{15}$ is interfered with $d_{16}$, which is undesirable in practical applications (e.g., hydrophones). It was reported that the coefficient $d_{16}$ can be eliminated by rotating the coordinate system around the $\mathrm{X}$ axis, without sacrificing the high value of $d_{15}$, with the rotation angle being determined by ${ }^{251,252}$

$$
\alpha=\arctan \left(\frac{d_{16}}{d_{15}}\right) .
$$

\section{Composition dependent properties}

It has been reported that the properties of relaxor-PT crystals strongly depend on the composition, especially in proximity to the MPBs. ${ }^{116,185,186,193,238,239,256-260}$ From thermodynamic analysis, the shear piezoelectric response of single domain crystals will increase as the composition approaches an MPB. ${ }^{261,262}$ As a consequence, the 
longitudinal dielectric and piezoelectric coefficients of domain engineered relaxor-PT crystals exhibit strong compositional dependent properties, as shown in Figure 15. For [001] and [011] poled R crystals, the piezoelectric coefficients $d_{33}$, $d_{31}, d_{32}$, and dielectric permittivity $\varepsilon_{33}$ were found to increase as the composition approached an MPB. In [001] poled PMN- $x$ PT crystals, the coefficient $d_{33}$ increased from $1200 \mathrm{pC} / \mathrm{N}$ to $2500 \mathrm{pC} / \mathrm{N}$ as $x$ increased from 0.26 to 0.30 . The coefficient $-d_{32}$ of [011] poled R crystals was found to increase from $1000 \mathrm{pC} / \mathrm{N}$ to $1800 \mathrm{pC} / \mathrm{N}$ as the composition approached the R-M/O phase boundary. In contrast, the electromechanical coupling factors were found to be relatively insensitive to compositional variations, with $k_{33}$ values being in the range of $0.89-0.93$.

Generally, the dielectric loss of relaxor-PT crystals exhibits maximum values around MPBs. For [001] poled R PINPMN-PT crystals, the dielectric loss factor increased to $\sim 1.2 \%$ for compositions close to the $\mathrm{R}-\mathrm{M} / \mathrm{O}$ phase boundary, while it was around $\leq 0.5 \%$ for all other compositions. ${ }^{116}$ The enhancement of loss factor is attributed to the ease of polarization rotation, which will be discussed in detail in Sec. IV B.

\section{F. Pyroelectric and electro-optic properties}

The ultrahigh dielectric and piezoelectric properties of relaxor-PT crystals have generally been the main focus, while other properties, including pyroelectric and electro-optic properties, have only been given attention in last few years. The pyroelectric coefficients of relaxor-PT crystals exhibit maximum values along their polar directions. ${ }^{263}$ Investigations of the pyroelectric properties for relaxor-PT crystals has generally focused on the single domain crystals (poled along polar direction). Table VII lists reported pyroelectric coefficients for relaxor-PT crystals. ${ }^{264-270}$ For [111] poled rhombohedral and [001] poled tetragonal relaxor-PT crystals, the pyroelectric coefficients $p$ are found to be on the order of $8 \sim 15$ $\times 10^{-4} \mathrm{C} / \mathrm{m}^{2} \mathrm{~K}$, being much higher than those of conventional pyroelectric crystals, such as triglycine sulfate (TGS) $\left(5.5 \times 10^{-4} \mathrm{C} / \mathrm{m}^{2} \mathrm{~K}\right)$ and $\mathrm{LiTaO}_{3}\left(2.3 \times 10^{-4} \mathrm{C} / \mathrm{m}^{2} \mathrm{~K}\right)$. The dielectric constant $(500 \sim 1000)$ and dielectric loss $(0.1 \%$ $\sim 0.4 \%$ ) of relaxor-PT crystals, however, are also much larger than those of TGS and $\mathrm{LiTaO}_{3}$. Therefore, in respect to the
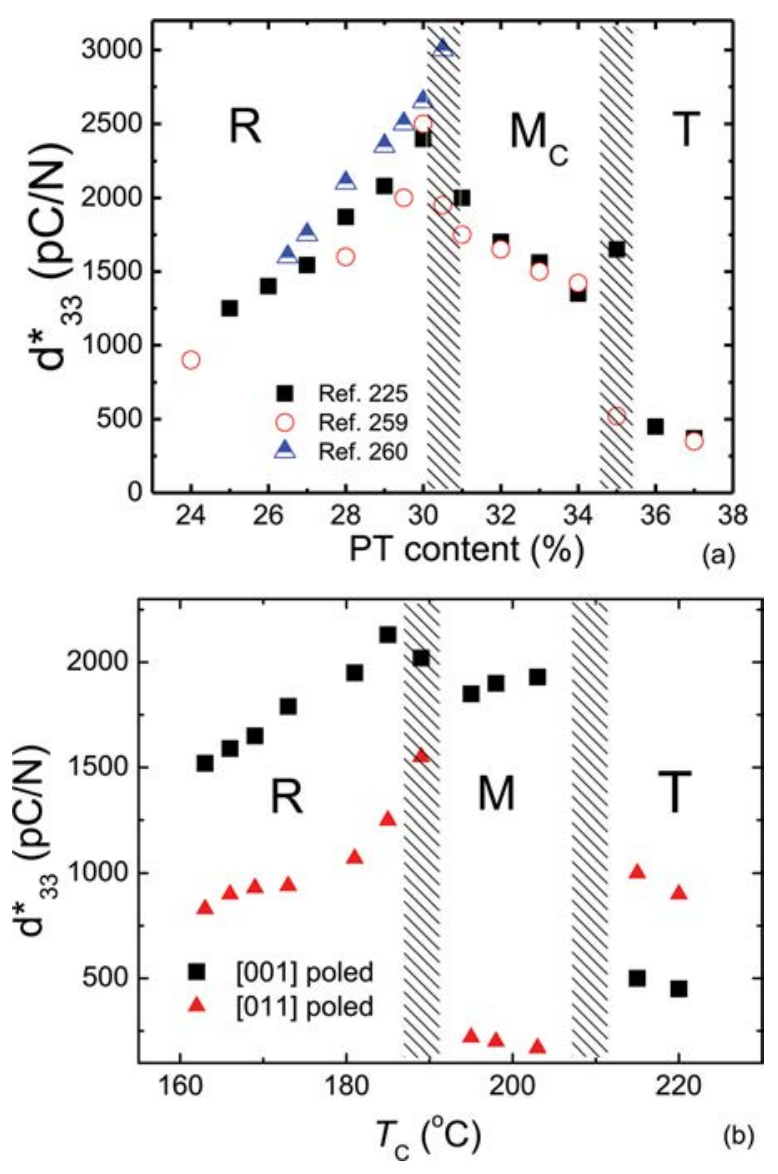

FIG. 15. (Color online) Composition dependence of piezoelectric response for (a) [001] poled PMN- $x$ PT and (b) [001] and [011] poled PIN-PMN-PT crystals. $116,225,259,260$

figure of merit (FOM, e.g., $F_{d} \propto \mathrm{p} /\left(\varepsilon_{0} \varepsilon_{r} \tan \delta\right)^{1 / 2}$ ), relaxorPT crystals do not offer obvious advantages to TGS and $\mathrm{LiTaO}_{3}$. In order to utilize relaxor-PT crystals for thermal imaging applications, crystals have been modified using Mn dopants and reported to effectively reduce both dielectric constant and loss, resulting in the improved FOMs, as listed in Table VII. ${ }^{268-270}$

Analogous to the piezoelectric response, the third-order tensor electro-optic coefficient $r_{33}$ of relaxor-PT crystals has been reported to be much higher along nonpolar directions

TABLE VII. Pyroelectric properties and FOMs for relaxor-PT crystals.

\begin{tabular}{|c|c|c|c|c|c|c|c|c|c|}
\hline Composition & $\mathrm{T}_{\mathrm{C}}\left({ }^{\circ} \mathrm{C}\right)$ & $\mathrm{T}_{\mathrm{RT}}\left({ }^{\circ} \mathrm{C}\right)$ & $p\left(10^{-4} \mathrm{C} / \mathrm{m}^{2} \mathrm{~K}\right)$ & $\varepsilon_{\mathrm{r}}(1 \mathrm{kHz})$ & $\tan \delta(1 \mathrm{kHz})$ & $F_{i}\left(10^{-10} \mathrm{~m} / \mathrm{V}\right)$ & $F_{v}\left(\mathrm{~m}^{2} / \mathrm{C}\right)$ & $F_{d}\left(10^{-5} \mathrm{~Pa}^{-1 / 2}\right)$ & Refs. \\
\hline $\mathrm{LiTaO}_{3}$ & 620 & - & 2.3 & 47 & 0.0005 & 0.72 & 0.17 & 15.76 & 264 \\
\hline$(\mathrm{SrBa}) \mathrm{Nb}_{2} \mathrm{O}_{6}$ & - & - & 5.5 & 400 & 0.003 & 2.35 & 0.07 & 7.2 & 264 \\
\hline PZT ceramic & 340 & - & 3.3 & 714 & 0.018 & 1.22 & 0.02 & 1.15 & 264 \\
\hline TGS & - & - & 5.5 & 55 & 0.025 & 2.12 & 0.43 & 6.1 & 264 \\
\hline PMN-0.26PT & 121 & 92 & 15.3 & 643 & 0.0028 & 6.1 & 0.11 & 15.3 & 265 \\
\hline PMN-0.29PT & 135 & 103 & 12.8 & 515 & 0.0063 & 5.25 & 0.11 & 9.8 & 266 \\
\hline PMN-0.37PT & 173 & -5 & 8.7 & 570 & 0.0041 & 3.5 & 0.07 & 7.6 & 265 \\
\hline PMN-0.28PT & - & - & 10.8 & 660 & 0.003 & - & - & - & 263 \\
\hline PZN-0.08PT & - & - & 8.2 & 950 & 0.003 & - & - & - & 263 \\
\hline PIMNT(42/30/28) & 187 & 152 & 9 & 702 & 0.002 & 3.6 & 0.06 & 10.2 & 267 \\
\hline Mn-PMN-0.26PT & 120 & 90 & 17.2 & 660 & 0.0005 & 6.88 & 0.12 & 40.2 & 268 \\
\hline Mn-PMN-0.29PT & 135 & 105 & 16.2 & 688 & 0.0005 & 6.48 & 0.11 & 37.1 & 269 \\
\hline Mn-PMN-0.38PT & 175 & - & 6.9 & 490 & 0.0026 & 2.76 & 0.06 & 8.2 & 270 \\
\hline
\end{tabular}


than that along the polar direction. For [001] poled T crystals and [111] poled R crystals, the $r_{33}$ coefficients were reported to be $70 \sim 130 \mathrm{pm} / \mathrm{V}$, while the $r_{33}^{*}$ was up to $300 \mathrm{pm} / \mathrm{V}$ for [001] poled $\mathrm{R}$ crystals $\left(\sim 30 \mathrm{pm} / \mathrm{V}\right.$ for $\left.\mathrm{LiNbO}_{3}\right) .{ }^{271-273}$ The high $r_{33}^{*}$ values are believed to be associated with the high $r_{15}$ of relaxor-PT crystals. The low optical transmission of relaxor-PT crystals, however, limits the potential O-E applications, due to the complex domain structure. It is known that domain wall density of relaxor-PT crystals can be minimized by poling along their respective polar directions. As reported, the transmission of [001] oriented tetragonal PMNPT crystals can be enhanced by $\sim 15 \%$ after poling. ${ }^{274}$

\section{ORIGIN OF PIEZOELECTRIC RESPONSE AND LOSSES}

\section{A. Piezoelectric properties}

The origin of the high piezoelectric response for relaxor-PT crystals has been debated since their discovery. $3,4,74,75,231,238,246-248,275-285$ From experimental observations, the mechanism of the ultrahigh piezoelectric activity in relaxor-PT crystals is different from that in polycrystalline piezoelectric ceramics. Of particular interest is that ultrahigh piezoelectric coefficients, low dielectric loss and strain-electric field hysteresis, being contradiction in PZT ceramics, occur simultaneously in domain engineered relaxor-PT crystals. ${ }^{3}$

Prior to the discovery of relaxor-PT, the highest piezoelectricity was found in PZT and related ternary ceramics for the compositions around their respective MPBs, where the piezoelectric coefficients were found to be on the order of $200-700 \mathrm{pC} / \mathrm{N}^{1,286}$ The high piezoelectric response of PZT ceramics is believed to originate from the MPB, where poling and domain wall mobility are enhanced. ${ }^{1}$ It is well known that the piezoelectricity of PZT ceramics can be tuned using various dopants, where donor dopants (e.g., $\mathrm{Nb}^{5+}$ and $\mathrm{Sb}^{5+}$ on $\mathrm{Ti}^{4+}$ site or $\mathrm{La}^{3+}$ on $\mathrm{Pb}^{2+}$ site) give rise to "soft" behavior, while acceptor dopants (such as $\mathrm{Fe}^{3+}$ and $\mathrm{Mn}^{3+, 2+}$ on $\mathrm{Ti}^{4+}$ site) lead to "hard" characteristics. The high piezoelectric response $\left(d_{33}>500 \mathrm{pC} / \mathrm{N}\right)$ in "soft" PZT ceramics is the result of enhanced domain wall mobility, which is a nonlinear and hysteresis process, accompanied by high dielectric/piezoelectric loss. In contrast, the domain wall motion of "hard" PZT ceramics is stabilized by the development of internal bias field, induced by acceptor dopants and the associated oxygen vacancies, leading to a relatively low level of piezoelectric activity and hysteresis.

In this section, concepts recently developed to explain the high piezoelectric activity in relaxor-PT crystals are surveyed, with the roles of MPBs, monoclinic phases, and relaxor component discussed in relation to experimental results.

\section{Electric field induced phase transitions}

In the middle $1990 \mathrm{~s}$, Park and Shrout ${ }^{3,61,62}$ published a number of papers that introduced the potential merits of relaxor-PT based crystals, reporting that the high E-field induced strains along [001] direction was associated with the E-field induced rhombohedral-tetragonal phase transition. As depicted in Figure 16, the spontaneous polarization of rhombohedral crystals along $\langle 111\rangle$ directions will incline to the [001] direction with increasing electric field and ultimately inducing a structural transition to the tetragonal phase. This predicted phase transition was confirmed by in situ high energy XRD techniques. ${ }^{287}$ Due to the lack of domain wall motion in this process (prior to the phase transition), hysteresis-free strain was observed. The underlying origin of the high piezoelectric coefficient, however, remained unknown.

\section{Polarization rotation mechanism}

In 2000, Fu and Cohen ${ }^{4}$ analyzed the free energy profile of PZN crystals using the first-principles linearized augmented plane wave (LAPW) method and proposed a polarization rotation mechanism to explain the high piezoelectric activity in relaxor-PT based crystals. ${ }^{4}$ In their work, the free energy under zero electric field (U) was calculated as a function of the spontaneous polar direction, as shown in Figure 17. Under an electric field, the total free energy of crystals can be expressed as F $=\mathrm{U}-\mathrm{P} \cdot \mathrm{E}$. It can be seen that the $-\mathrm{P} \cdot \mathrm{E}$ term decreases as the angle between $\mathrm{P}$ and $\mathrm{E}$ decreases, meaning that the applied electric field stabilizes the ferroelectric phase whose polar direction lies close to the applied electric field. With the application of an [001] electric field, two polar rotation paths of PZN crystals, i.e., $\mathrm{a} \rightarrow \mathrm{f} \rightarrow \mathrm{g} \rightarrow \mathrm{e}$ and $\mathrm{a} \rightarrow \mathrm{b} \rightarrow \mathrm{c} \rightarrow \mathrm{d} \rightarrow \mathrm{e}$, were given, as depicted in Figure 17(a). In the low-field region, the piezoelectric
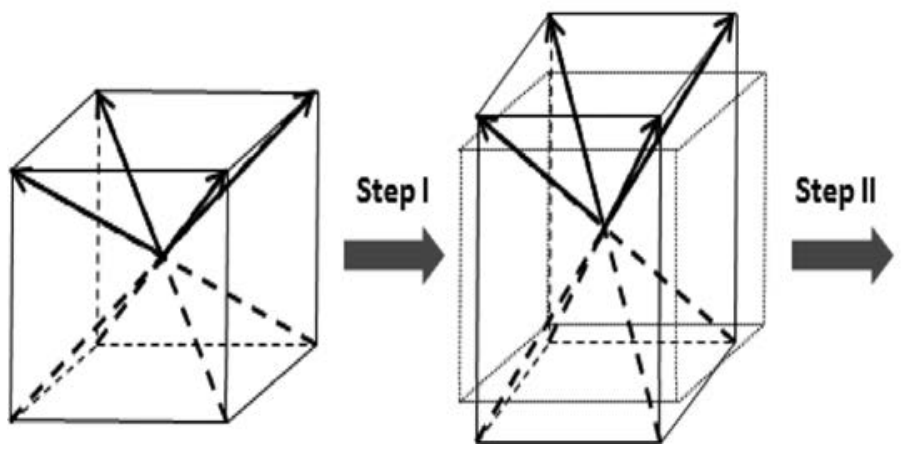

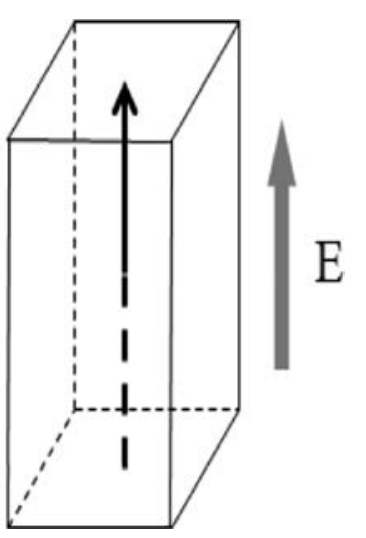

FIG. 16. Variation of polarization for rhombohedral relaxor-PT crystals under [001] electric field. 

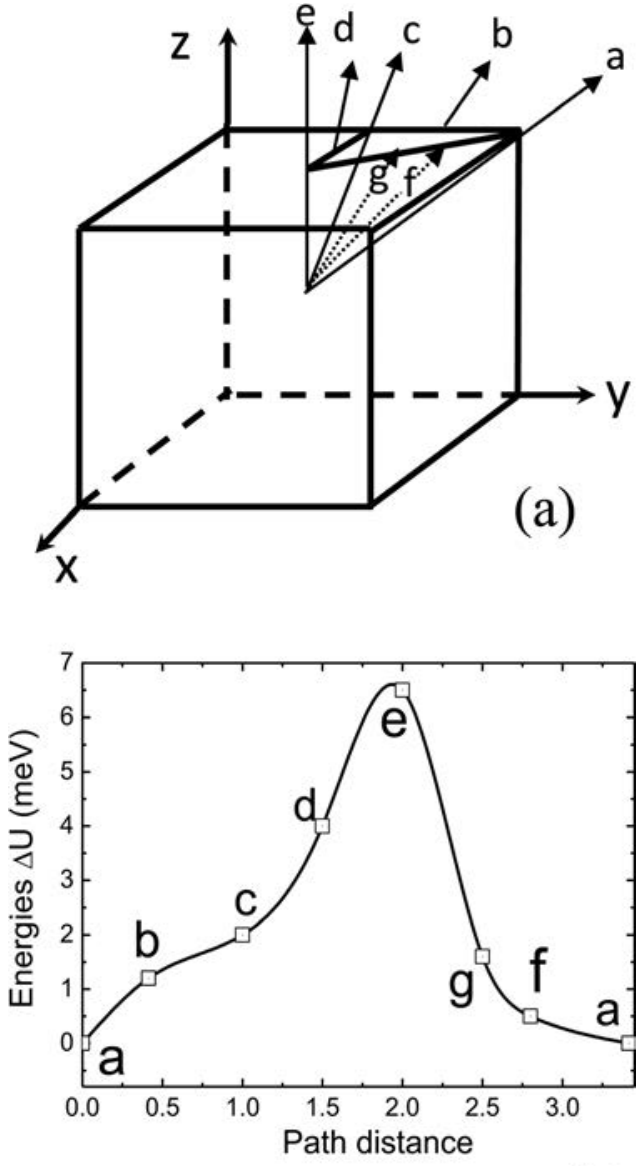

(b)

FIG. 17. Polarization rotation path (a) and related free energy (b).Reprinted by permission form H. Fu and R. E. Cohen, Nature 403, 281 (2000). Copyright $($ ) 2000, Nature Publishing Group.

coefficient of the first path was calculated to be five times larger than that of the second one, because the $\mathrm{a} \rightarrow \mathrm{f}$ path was much flatter than $\mathrm{a} \rightarrow \mathrm{b}$ path, where a flatter energy profile corresponds to more structural instability, indicating that a small electric field will induce a large polar rotation, resulting in high level of E-field induced strain. ${ }^{277}$ Obviously, a flat free energy is the dominant factor for obtaining high piezoelectric response.

"Polarization rotation" vs "domain wall motion". As mentioned above, the facilitated "polarization rotation" through the flattened free energy is thought to be critical for achieving the high piezoelectric response in relaxor-PT crystals. However, the nomenclature "polarization rotation" should be distinguished from "domain wall motion (or domain switching)," which is generally used to explain the contribution to the piezoelectric response in polycrystalline ceramics.

Domain wall motion is the phenomenon where ferroelectric materials change from one spontaneously polarized state to another under electric or mechanical field, in order to minimize the free energy, which can be deemed as a rotation of the lattice in space $\left(90^{\circ}\right.$ rotation in tetragonal crystals, $71^{\circ}$ and $109^{\circ}$ in rhombohedral one, and $60^{\circ}, 120^{\circ}$, and $90^{\circ}$ in orthorhombic one), without a change in lattice symmetry (no lattice distortion). During domain wall motion, only a part of the lattice of one domain switches to another stable state, as shown in Figure 18(a). In addition, domain wall motion can be separated into two types: reversible and irreversible. Figure 18(b) shows a schematic figure of the free energy with respect to the domain wall position in polycrystalline ceramics, where the irreversible domain wall motion refers to a motion over one or several energy barriers, while reversible domain wall motion corresponds to motion among two energy barriers. ${ }^{36}$

In contrast to domain wall motion, the polarization rotation process is induced by application of a perpendicular electric field, leading to a shear deformation of the lattice (lattice distortion), which induces variation of the lattice symmetry. During the polarization rotation process, the lattice distortion in each domain variant is identical and thought to be reversible, as shown in Figure 17, where no energy barrier exists in the polarization rotation path. Admittedly, firstorder ferroelectric phase transitions can be observed at high levels of electric field for relaxor-PT crystals with MPB compositions, being related to an irreversible polarization rotation process. For analysis of the piezoelectric coefficient in crystals, however, only polarization rotation at low levels of electric fields, being deemed as a reversible process, need to be concerned.

Considering the contribution to the longitudinal piezoelectricity, the polarization rotation mechanism refers to a contribution from the shear deformation of the lattice (originate from lattice distortion) is the intrinsic effect. However, the contribution of domain wall motion to the piezoelectricity is based on the anisotropy of the lattice scale and the

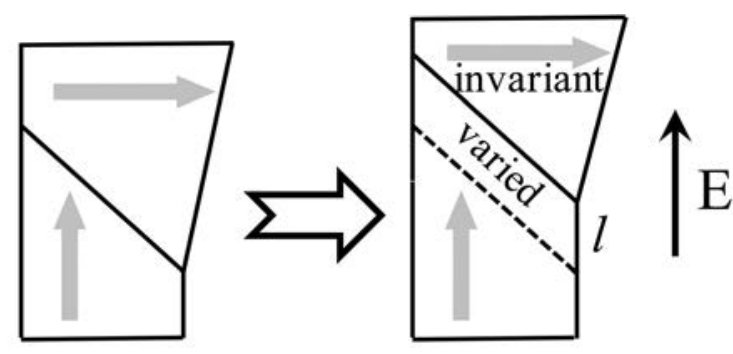

(a)

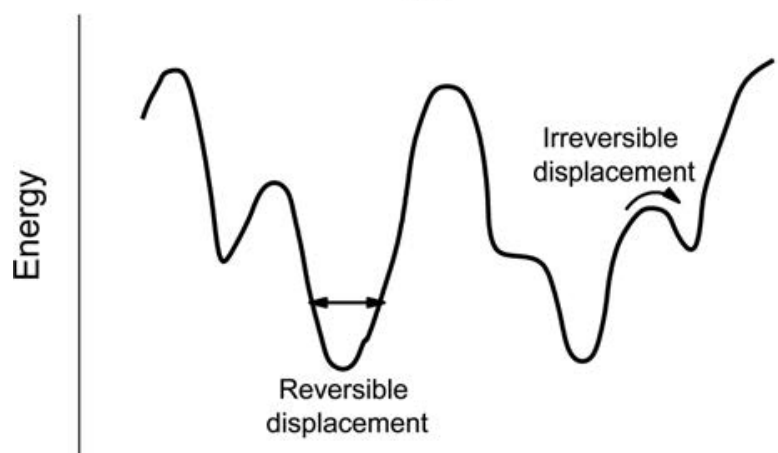

Domain wall position

(b)

FIG. 18. (a) $90^{\circ}$ domain wall motion upon electric field and (b) Schematic of free energy with respect to domain wall position. ${ }^{36}$ 
contribution to strain comes from lattice rotation, where no lattice distortion is involved. Taking tetragonal domains as an example, the contribution of domain wall motion to electric-field-induced-strain is mainly due to the lattice parameters $c>a$. Obviously, if $c=a$, the domain wall motion shown in Figure 18(a) cannot contribute to the piezoelectric response. Thus, domain wall motion is not associated with the piezoelectric distortion of lattice and belongs to an extrinsic effect.

\section{High shear piezoelectric response and MPB}

The piezoelectric matrix of single domain relaxor-PT crystals has been reported, ${ }^{72,112,115,243}$ based on which the piezoelectric anisotropy has been extensively investigated. $^{276,277}$ The longitudinal piezoelectric coefficients are found to exhibit very strong orientation dependence due to the high levels of $d_{15}\left(d_{15} \gg d_{33}\right) .^{243,246-248}$ For rhombohedral relaxor-PT crystals, therefore, the high longitudinal coefficient $d_{33}^{*}$ along the [001] direction is attributed to the high shear piezoelectric response in the single domain state. Based on a thermodynamic approach, Damjanovic et al. discussed the origin of high shear piezoelectric response for ferroelectric materials. ${ }^{276,277}$ The shear piezoelectric coefficient is in proportion to the transverse dielectric permittivity, spontaneous polarization, and electrostrictive constant, following $d_{15} \propto \varepsilon_{0} \varepsilon_{11} \mathrm{Q}_{55} \mathrm{P}_{3}$, where $P_{3}$ is the spontaneous polarization, $Q_{55}$ is the electrostrictive constant, and $\varepsilon_{11}$ is the transverse dielectric permittivity. Compared with the spontaneous polarization and electrostrictive constant, the high level of transverse dielectric permittivity was believed to be responsible for the high shear piezoelectric coefficient. For perovskite ferroelectric crystals, the transverse dielectric permittivity mainly arises from a polarization rotation process, as depicted in Figure 19, thus the shear deformation can be softened by flattening the polarization rotation path (enhancing the structural instability). Based on reported results, the shear piezoelectric coefficients $d_{15} / d_{24}$ can be significantly enhanced at compositions near MPBs or at temperatures close to polymorphic phase transitions (PPTs), where phase instability is due to similar free energies of the various morphotropic or polymorphic phases.

For an explanation of the high longitudinal piezoelectric response in domain engineered relaxor-PT crystals, both polarization rotation and high shear piezoelectric mechanisms are actually based on the same physical foundation,

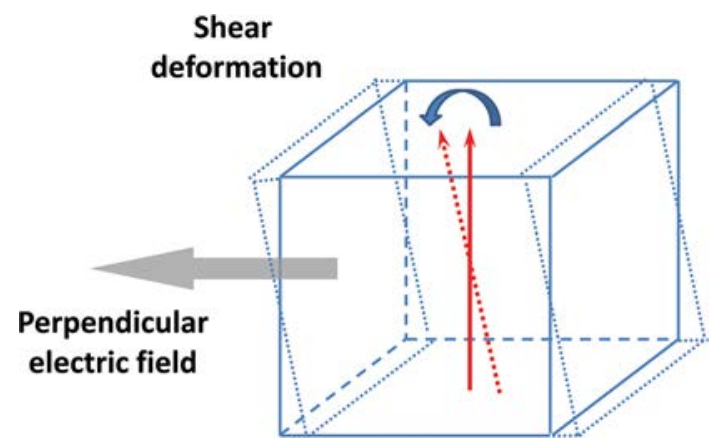

FIG. 19. (Color online) Shear piezoelectric deformation and polarization rotation. i.e., flat free energy profile or structural instability. Thus, the high longitudinal piezoelectric response can be observed at compositions close to MPBs, where the free energy profile is flat. However, other ferroelectric crystals, such as $\mathrm{Pb}(\mathrm{Zr}, \mathrm{Ti}) \mathrm{O}_{3}$, $(\mathrm{K}, \mathrm{Na}) \mathrm{NbO}_{3}$, and $\mathrm{BaTiO}_{3}(\mathrm{BT})$, with peak piezoelectric properties at their respective MPBs or PPTs, being much lower when compared to relaxor-PT crystals, leave the question of which factor induces the high level of structural instability or flat free energy profile in relaxor-PT crystal systems.

\section{The role of a monoclinic phase}

Prior to the discovery of monoclinic phases, only rhombohedral, orthorhombic, and tetragonal phases were experimentally observed in perovskite ferroelectrics, and only these three ferroelectric phases were proved to be stable by six-order Devonshire thermodynamic analysis. ${ }^{27}$ The different types of monoclinic phases, namely, $\mathrm{M}_{\mathrm{A}}, \mathrm{M}_{\mathrm{B}}$, and $\mathrm{M}_{\mathrm{C}}$ (as shown in Figure 20, where polar vector of $\mathrm{M}_{\mathrm{A}}$ phase lies in (11) plane, while polar vectors of $\mathrm{M}_{\mathrm{B}}$ and $\mathrm{M}_{\mathrm{C}}$ lie in (101) and (010) planes, respectively), have been observed by various research groups using in situ high energy X-ray/neutron and polarized light microscopy ${ }^{276,279-284}$ and also theoretically confirmed to be stable by higher-order Devonshire theory. ${ }^{284}$

The monoclinic phase is believed to act as a "structural bridge" between tetragonal, orthorhombic, and/or rhombohedral phases, consequently, the polarization rotation in the $\mathrm{M}$ phase is thought to be a critical factor for the high piezoelectric property of relaxor-PT crystals. ${ }^{231}$ Recently, Viehland et al. ${ }^{285,288}$ proposed that the observed monoclinic phase is an adaptive phase, which is formed by tetragonal microdomains with very low domain wall energy. In this model, $90^{\circ}$ domain wall motion was thought to be the dominate factor for the observed high piezoelectricity. In addition, the hysteresis-free S-E loops of relaxor-PT crystals were also explained by the adaptive phase model, being attributed to the very low domain wall energy of the fine microdomains. $^{285,288}$ However, several researchers hold critical opinions in the understanding of the high piezoelectricity from monoclinic phases. ${ }^{5,155,289}$ Kisi et al. ${ }^{289}$ proposed that it is the high piezoelectricity that induces monoclinic phase in PZN-PT crystals and not the monoclinic phase

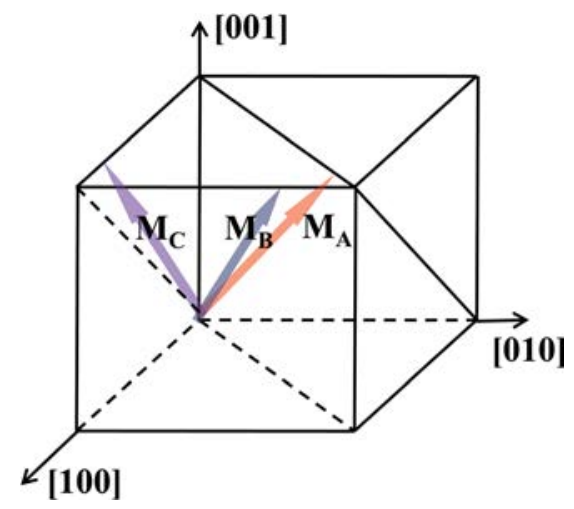

FIG. 20. (Color online) Various monoclinic phases for relaxor-PT crystals. 
contributing to the high piezoelectricity. Thus, a monoclinic phase can be recognized as a monoclinic distortion induced by strong piezoelectric anisotropy in relaxor-PT crystals.

\section{The role of relaxor end member}

Relaxors, including PMN and PZN, have been extensively studied for more than half century. ${ }^{50,54}$ However, the relaxor characteristics have not been involved in the explanation of the high piezoelectric properties in the analyses above. We should bear in mind that the piezoelectric activity of relaxor-PT ferroelectrics is much higher than those of classical ferroelectric crystals (e.g., BT and PZT). According to the random-cation model, relaxors PMN and PZN consist of local randomly oriented polar cluster, referred to as polar nanoregion (PNR), being chemically ordered (Nb:Mg/ $\mathrm{Zn}=2: 1$ ) and embedded in a disordered matrix. ${ }^{290}$ The polar nanoregions are one of the key factors for relaxor ferroelectrics, which can significantly affect the macroscopic properties. $^{51}$ In 2008, Xu et al. ${ }^{278,291}$ studied relaxor-PT crystals using neutron inelastic scattering experiments and found that the transverse acoustic (TA) mode softened, being attributed to the existence of PNRs, which interact with the propagation of phonons, giving rise to phase instability. This soft TA mode is related to an ease of shear deformation, indicating a high shear piezoelectric response of relaxor-PT crystals. According to this work, the relationship between high piezoelectric response and relaxors was established, helping to partially answer the question "why high piezoelectric response was only observed in relaxor based crystals other than classical ferroelectric crystals."

\section{Critical factors for high piezoelectricity}

Various mechanisms corresponding to the high piezoelectric activity in relaxor-PT crystals have been discussed above. Concept 1 helped us to recognize the high strain and hysteresis-free S-E loops for domain engineered relaxor-PT crystals. Concepts 2 and 3 demonstrated that the dominant factor for the high piezoelectric response was a flattened free energy profile, which can be induced by structural instability, e.g., around MPB or PPT. Concept 4 proposed that the monoclinic phase was the dominant factor resulting in high piezoelectricity, where a monoclinic phase was thought to be a bridge facilitating polarization rotation or adaptive phase (strong domain mobility contribution to piezoelectricity). Concept 5 built a relationship between the structural instability and relaxors for relaxor-PT systems, which can actually be deemed as the addition to concepts 2 and 3, being responsible for the high piezoelectricity observed in relaxor-PT crystals.

In order to delineate the critical factors responsible for ultrahigh piezoelectricity in relaxor-PT crystals, the related properties of [001] poled PMN-xPT crystals were analyzed as a function of composition and ferroelectric phase.

The sub-coercive field piezoelectric behavior of PMNPT crystals was studied using the Rayleigh approach, as shown in Figure 21, where $d_{\text {init }}$ and $\alpha \mathrm{E}_{0} /\left(\alpha \mathrm{E}_{0}+\mathrm{d}_{\text {init }}\right)$ represents the degree of reversible (hysteresis-free) piezoelectric contribution and irreversible contribution. ${ }^{225}$ Due to a stable domain engineered structure, the irreversible contribution is generally less than $10 \%$ for either R or M crystals, as shown in Figure 21(b), demonstrating that the intrinsic contribution (lattice) is mainly responsible for the high piezoelectric response. Of particular interest is that crystals with $\mathrm{M}$ phase show no piezoelectric advantage over the $\mathrm{R}$ phase, revealing that an $\mathrm{M}$ phase is not necessary for an ease polarization rotation process. The enhanced piezoelectricity for PMNxPT crystals is observed for compositions in proximity to the MPBs and not in $\mathrm{M}$ phase region. The monoclinic phases play the role in formation of MPBs with $\mathrm{R}$ and/or $\mathrm{T}$ phases, inducing phase instability and lead to the high piezoelectric response of relaxor-PT crystals. ${ }^{225}$

To further understand the enhancement of piezoelectricity, the electric field induced strain (at $70 \mathrm{kV} / \mathrm{cm}$ ) and the ferroelectric phase transition E-field $(\mathrm{R} / \mathrm{M} \rightarrow \mathrm{T}$ phase) for [001] oriented PMN-xPT crystals were measured, as shown in Figure 22. For the $\mathrm{R}$ phase, the strain was on the order of $0.7 \%$ and nearly independent of composition, whereas the phase transition E-field was found to decrease as the composition approached the R-M phase boundary. According to the polarization rotation mechanism, the decrease of phase transition E-field demonstrates a flat free energy profile, leading
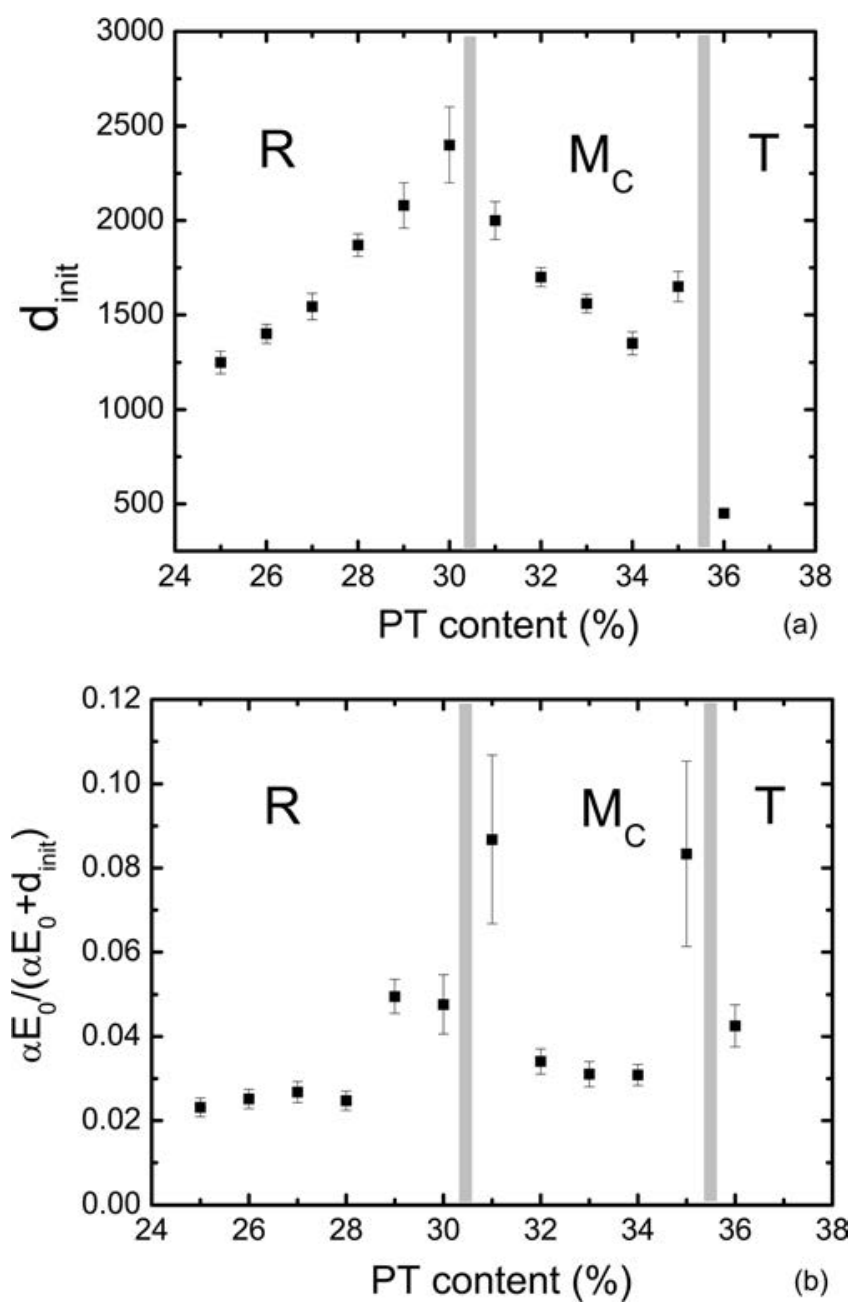

FIG. 21. Compositional dependence of Rayleigh parameters for PMN-xPT crystals at $1 \mathrm{~Hz}$ : (a) reversible contribution $\mathrm{d}_{\text {init }}$ and (b) irreversible contribution. Reprinted with permission from F. Li et al., Journal of Applied Physics 108, 034106 (2010). Copyright (C 2010, the American Institute of Physics. 


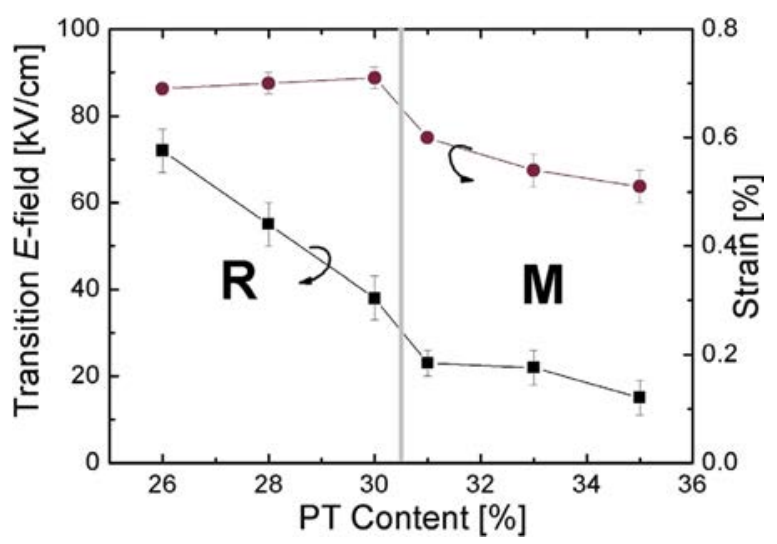

FIG. 22. (Color online) Transition electric fields and the electric field induced strain levels at $70 \mathrm{kV} / \mathrm{cm}$ for PMN-xPT crystals.

to high piezoelectricity. In the $\mathrm{M}$ phase region, the piezoelectric coefficient was observed to first decrease and then increase with increasing PT (Figure 21(a)), while both the strain and phase transition E-field decreased with increasing PT (Figure 22), which can be explained by considering the variation of both free energy profile and spontaneous polarization direction as a function of composition. ${ }^{225}$

The relaxor behavior of PMN-xPT crystals was analyzed using a modified Curie law

$$
\frac{1}{\varepsilon}-\frac{1}{\varepsilon_{m}}=\frac{\left(T-T_{m}\right)^{\gamma}}{C},
$$

where $\varepsilon_{\mathrm{m}}$ is the maximum value of the dielectric permittivity at $T_{m}, C$ is the Curie-like constant, and $\gamma(=1 \sim 2)$ is the degree of diffuseness. A higher value of $\gamma$ represents a higher level of relaxor behavior. For classical ferroelectrics, the value of $\gamma$ is 1 , while for relaxor, the value is 2 .

The measured $\gamma$ values for PMN-xPT crystals are shown in Figure 23, with the highest value being on the order of 1.85 for $x=0.31$. Of particular interest is that the factor $\gamma$ shows very high values $(>1.7)$ and is nearly compositionally independent in the $\mathrm{R}$ phase region, however, it drastically decreases with increasing PT content in $\mathrm{M}$ and $\mathrm{T}$ phase regions, with the values being $<1.3$ for the $\mathrm{T}$ phase. Based on concept 5, the relaxors PMN and PZN can induce phase instability, giving rise to high piezoelectricity. Therefore, if a higher value of $\gamma$ represents an enhanced relaxor effect on phase instability, the fact that the piezoelectricity of R relaxor-PT crystals far outperforming tetragonal ones, can be explained.

In summary, the measured piezoelectric response of PMN-xPT crystals as a function of composition can be well explained by concepts 2, 3, and 5. A possible conclusion can be derived: phase instability is the most important factor for inducing a high piezoelectric response. To obtain a high level of phase instability in ferroelectric crystals, not only MPBs (or other coexist phases, such as PPTs) but also a relaxor component is required. For relaxor-PT crystals, the induced phase instability is generally associated with softening of a TA mode (high $d_{15}$ ) or facilitation of a polarization rotation process. In contrast to domain wall motion in PZT polycrystalline ceramics, the high and hysteresis-free piezoelectric response of relaxor-PT crystals is attributed to the

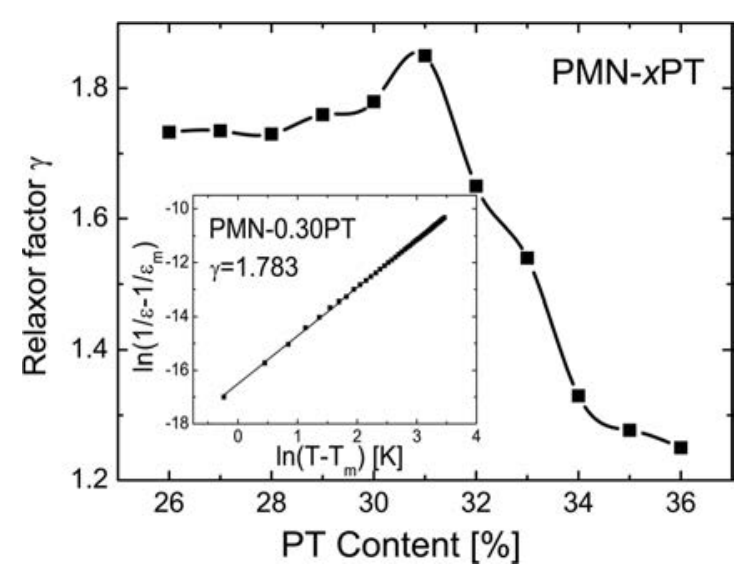

FIG. 23. Relaxor factor $\gamma$ for [001] oriented PMN-xPT crystals, small inset is the dielectric constant vs. temperature for PMN-0.30PT.

intrinsic/lattice contribution. Relaxor-PT crystals generally exhibit strong temperature dependent properties and high mechanical loss (see Secs. IV B and V), due to contribution of polarization rotation. Thus, enlarging the longitudinal piezoelectric response $\left(d_{33}\right)$ of single domain crystals may be a possible way to exploit new piezoelectric crystals with improved temperature stability and low mechanical loss. In this respect, Damjanovic ${ }^{292}$ proposed a special phase boundary system, where the high single domain coefficients $d_{15}$ and $d_{33}$ can be simultaneously obtained.

\section{B. Loss in relaxor-PT crystals}

\section{Internal bias and domain wall motion}

The dielectric and mechanical losses are primarily related to the domain wall motion in ferroelectric ceramics. In order to reduce loss in polycrystalline ceramics, the general approach has been to modify them with "acceptor" dopants, such as $\mathrm{Fe}^{3+, 2+}$ and $\mathrm{Mn}^{3+, 2+}$, which substitute for $\mathrm{Zr}^{4+} / \mathrm{Ti}^{4+}$ cations, resulting in defect dipoles of acceptor-oxygen vacancies. These dipoles align parallel to the polarization direction, which effectively clamp domain wall motion, leading to reduced dielectric and mechanical losses. ${ }^{1,2,19,20,136}$ For acceptor doped relaxorPT crystals (third generation, such as Mn:PMN-PZT), since there are no grain boundaries, the defect dipoles in the crystals must occupy energetically preferred sites in the lattice, forming anisotropic centers, locally or within a domain. After poling, the dipoles realign themselves along a preferential direction for the spontaneous polarization and move to the high stressed area of domain walls by diffusion, leading to an internal bias, pinning the domain walls, and stabilizing the domains. ${ }^{136,293}$ However, relaxor-PT crystals show strong anisotropic behavior, thus, the direction of the internal bias is not well-known and will behave differently when the crystals are poled along polar or nonpolar directions, further studies need to be carried out. It should be noted here that the domain size also affects the mechanical loss of the crystals, where the $Q_{m}$ was found to increase with increasing domain size. ${ }^{43}$

\section{Polarization rotation}

In contrast to polycrystalline ceramics, domain wall motion can be minimized in crystals with various domain 


\section{Rotation}

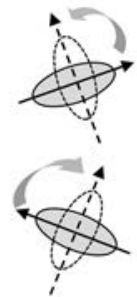

Discontinuous rotation (Domain switching)

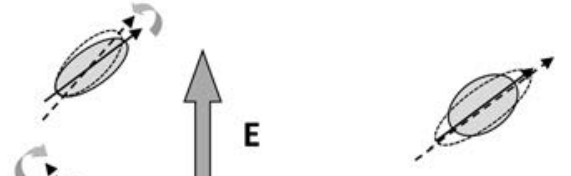

Continuous rotation

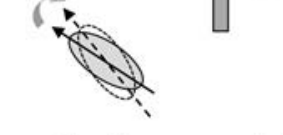

configurations, such as single domain states (poled along polar directions) and engineered domain configurations with equivalent domains ([001] poled rhombohedral crystals and [111] poled tetragonal crystals, with " $4 \mathrm{R}$ " and " $3 \mathrm{~T}$ " engineered domains), resulting in low dielectric loss. In spite of the absence of domain wall motion in domain engineered crystals, however, the level of mechanical loss observed is relatively high. As given in Table VI, ${ }^{250}$ the mechanical $Q_{m}$ (inverse of the mechanical loss) in single domain crystals is found to be much higher than those in domain engineered crystals. From these results, it was proposed that the high level of mechanical loss in domain engineered crystals (" $4 \mathrm{R}$ ") is associated with polarization rotation. It should be noted that both polarization rotation and domain wall motion are related to E-field induced polar direction variation, where the difference is that the former and latter processes refer to continuous and discontinuous variations, respectively, as shown in Figure 24. The low losses observed in hard piezoelectric ceramics and/or single domain crystals are due to the lack of E-field induced polar direction variation, where only polarization extension occurs (no polarization rotation involved) in single domain crystals due to the application of external field along the polar direction.

\section{Polarization rotation angle}

From the above, one can conclude that the losses of relaxor-PT crystals should be closely related to the polarization rotation process. In the following discussion, the losses are analyzed with respect to the angle between the polar and E-field directions, referred to as polarization rotation angle. ${ }^{38}$ Table VIII lists dielectric loss and mechanical quality factors for relaxor-PT crystals with various domain engineered structures and different vibration modes. Both the mechanical quality and dielectric quality factors (inverse of dielectric loss) were found to decrease with increasing polarization rotation angle. For the longitudinal mode, minimum
Extension

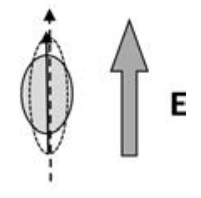

Single domain crystals
FIG. 24. (Color online) Schematic for polarization rotation and extension. The polarization rotation can be found in soft materials (soft PZT and domain engineered crystals); In hard ceramics and single domain crystals, polarization rotation is minimized, due to the internal bias field (hard ceramics) and main crystals). external field being along polar direction (single do-

mechanical and dielectric losses are found for " $1 \mathrm{~T}$ " and " $1 \mathrm{R}$ " crystals, where the polarization rotation angle is zero. For the shear mode case, the dielectric and mechanical losses, being $>1 \%$, are much higher than those of the longitudinal mode due to the large polarization rotation angle, being on the order of $90^{\circ}$. Thus, it can be concluded that a small polarization rotation angle corresponds to a low level of dielectric/mechanical losses for relaxor-PT crystals.

\section{Morphotropic phase boundary}

As discussed in Sec. IV A, ${ }^{225}$ the irreversible contribution is enhanced in relaxor-PT crystals as the composition approaches an MPB, due to the increased phase instability, leading to enhanced losses. In addition, the polarization rotation process becomes easier for compositions close to an MPB. As discussed in Sec. IV A 2, a facilitated polarization rotation was thought to be associated with high levels of loss. Due to these two factors, the mechanical quality factor was observed to decrease for compositions in proximity to MPBs, as evident in Figure 25. It should be noted here that the increase of structural/phase instability enlarges both piezoelectric/dielectric properties and the related losses.

\section{Losses under high ac drive field}

Other factors, including the electrode selection, surface finish of the samples, damping, etc., will also affect the measured mechanical quality factor, as previously mentioned in Sec. I B $1 \mathrm{f}$. Due to the nonlinear characteristics of ferroelectrics, however, such as domain wall motion, the measured losses strongly depend on the amplitude of the driving field. ${ }^{135,137,294-296}$ These measured high field values are referred to as large signal losses, to be separated from the small signal losses (measured at $\mathrm{V}_{\mathrm{rms}}<1 \mathrm{~V}$ ), which are generally reported in the references. Figure 26 shows the piezoelectric loss and mechanical loss (inverse of mechanical $Q_{m}$ ) as a function of the driving field amplitude, where both types

TABLE VIII. The loss/quality factors for relaxor-PT crystals as a function of polarization rotation angle.

\begin{tabular}{lccccc}
\hline \hline Domain configuration & Vibration mode & Polarization rotation angle & Mechanical quality factor & Dielectric loss & Piezoelectric coefficient (pC/N) \\
\hline $1 \mathrm{~T} / 1 \mathrm{R}$ & shear & $90^{\circ}$ & $<30$ & $1 \% \sim 2 \%$ & $>2000$ \\
$4 \mathrm{R}$ & longitudinal & $54.7^{\circ}$ & $100 \sim 200$ & $0.4 \% \sim 1 \%$ & $>1500$ \\
$4 \mathrm{O} / \mathrm{M}_{\mathrm{C}}$ & longitudinal & $\leq 45^{\circ}$ & $200 \sim 400$ & $0.2 \% \sim 1 \%$ & $>1500$ \\
$2 \mathrm{R}$ & longitudinal & $35.3^{\circ}$ & $500 \sim 800$ & $0.2 \sim 0.5 \%$ & $1000 \sim 1500$ \\
$1 \mathrm{~T} / 1 \mathrm{R}$ & longitudinal & $0^{\circ}$ & $>1000$ & $0.2 \%$ & $60 \sim 500$ \\
\hline \hline
\end{tabular}




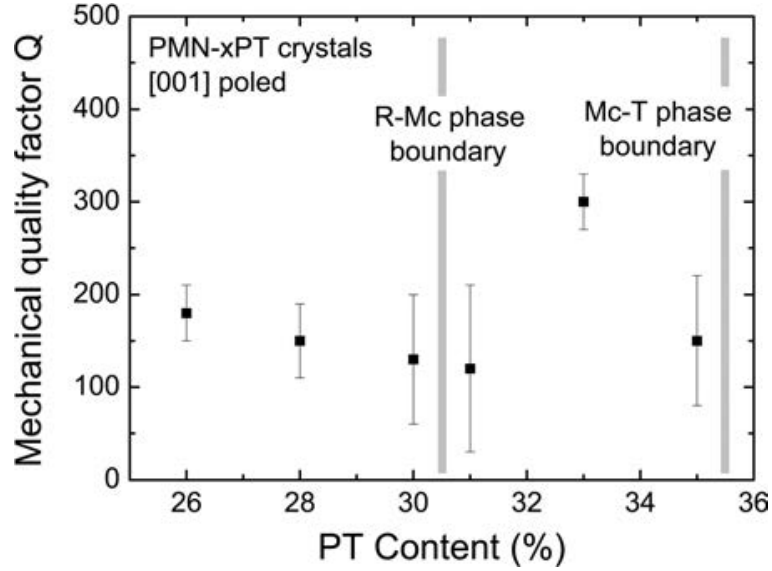

FIG. 25. Mechanical quality factor $Q_{m}$ vs. composition for PMN-xPT crystals.

of loss were found to increase with increasing drive field. According to Rayleigh analysis, ${ }^{36}$ the irreversible piezoelectric contribution will increase with increasing amplitude of electric field, responsible for the increase of loss factors.

\section{PROPERTIES UNDER EXTERNAL BOUNDARY CONDITIONS}

Piezoelectric relaxor-PT single crystals used in electromechanical devices may be subjected to various external conditions, including thermal, electric fields $(d c$ or $a c)$, mechanical stresses (uniaxial or hydrostatic pressure), or the combination of the above, which may significantly affect the crystal properties. Therefore, relaxor-PT crystals, as a new material of choice for high performance electromechanical applications, need to be investigated under various external conditions. In this section, the properties of relaxor-PT crystals are reviewed as a function of various boundary conditions.

\section{A. Temperature dependence}

The thermal stability of dielectric and piezoelectric properties is very important for most electromechanical applications, such as medical transducers and vector sensors, to name a few. Thus, it is desirable to understand the temperature usage range and thermal behavior of piezoelectric crystal elements. The temperature dependent properties of relaxor-PT crystals have been extensively studied, with most investigations focusing on [001] poled crystals. ${ }^{73,100,109-111,123,127-130,297-300}$ Figure 27 gives the longitudinal piezoelectric/dielectric response as a function of temperature for [001] poled relaxorPT crystals, showing similar temperature dependent behavior for different crystal compositions with the same phase. Generally, the piezoelectric and dielectric responses drastically degrade above $\mathrm{T}_{\mathrm{FF}}$, the ferroelectric phase transition temperature. Prior to $\mathrm{T}_{\mathrm{FF}}$, the temperature dependent behavior is explained in the following.

As discussed in Secs. III and IV, the high longitudinal piezoelectric coefficients of [001] poled $\mathrm{R}$ and $\mathrm{O} / \mathrm{M}$ crystals are attributed to the high shear piezoelectric response in single domain state, thus, the temperature dependence of longitudinal piezoelectricity is closely related to the temperature
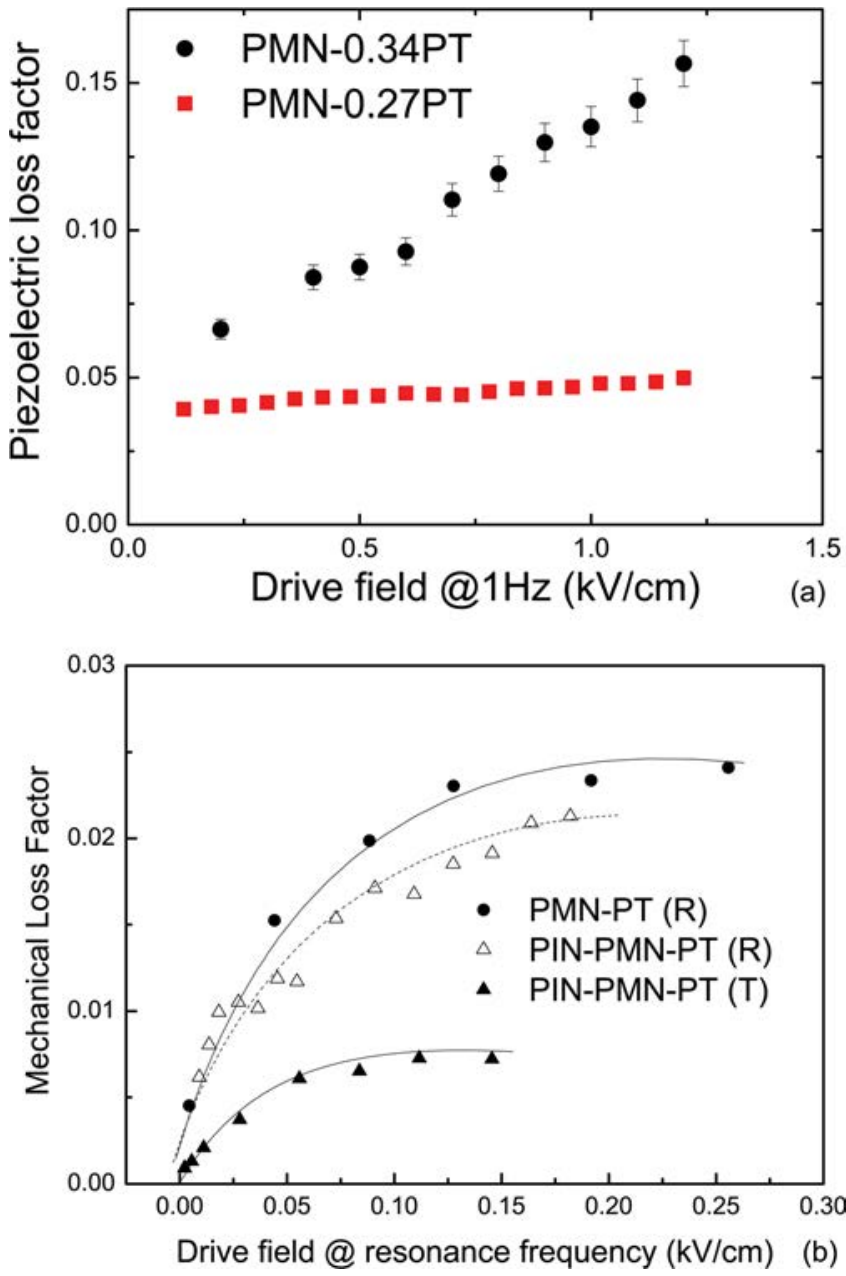

FIG. 26. (Color online) (a) Piezoelectric loss (determined by S-E loops) and (b) mechanical loss as function of drive field (data are from Refs.137 and 295).

dependent shear piezoelectric behavior, which is presented in Figure 28 using PIN-PMN-PT as the example. The measured temperature dependence of the shear piezoelectric behavior can be explained by analyzing the phase diagram and related polarization-rotation path, as shown in Figure 29. ${ }^{37,38}$ In this figure, the orthorhombic $\mathrm{O}$ phase is used instead of monoclinic $\mathrm{M}_{\mathrm{C}}$ phase, due to the fact that $\mathrm{M}_{\mathrm{C}}$ is a slightly distorted $\mathrm{O}$ phase. It should be noted that both $\mathrm{O}$ and $\mathrm{M}_{\mathrm{C}}$ symbols are used in this review, based on the references from various research groups. For rhombohedral crystals, upon applying a vertical electric field along $[11 \overline{2}]_{\mathrm{C}}$, the spontaneous polarization rotates from $[111]_{\mathrm{C}}$ to $[001]_{\mathrm{C}}$, giving rise to deviation in crystal symmetry from $3 \mathrm{~m}$ (rhombohedral phase) to $4 \mathrm{~mm}$ (tetragonal phase). This rotation becomes easier as the temperature approaches the R-T phase transition, responsible for the improved shear piezoelectric properties with increasing temperature. For T crystals, however, the spontaneous polarization rotates from $[001]_{\mathrm{C}}$ to $[011]_{\mathrm{C}}$ under a perpendicular electric field along $[010]_{\mathrm{C}}$, inducing crystal symmetry deviating from $4 \mathrm{~mm}$ to $\mathrm{mm} 2$. The crystals move away from the $\mathrm{T}-\mathrm{O}$ phase transition point with increasing temperature, leads to the decreased shear coefficient $d_{15}$. For $\mathrm{O}$ crystals, the scenario is complicated due to the anisotropic $\mathrm{mm} 2$ symmetry. There are two shear piezoelectric coefficients $d_{15}$ and $d_{24}$. 

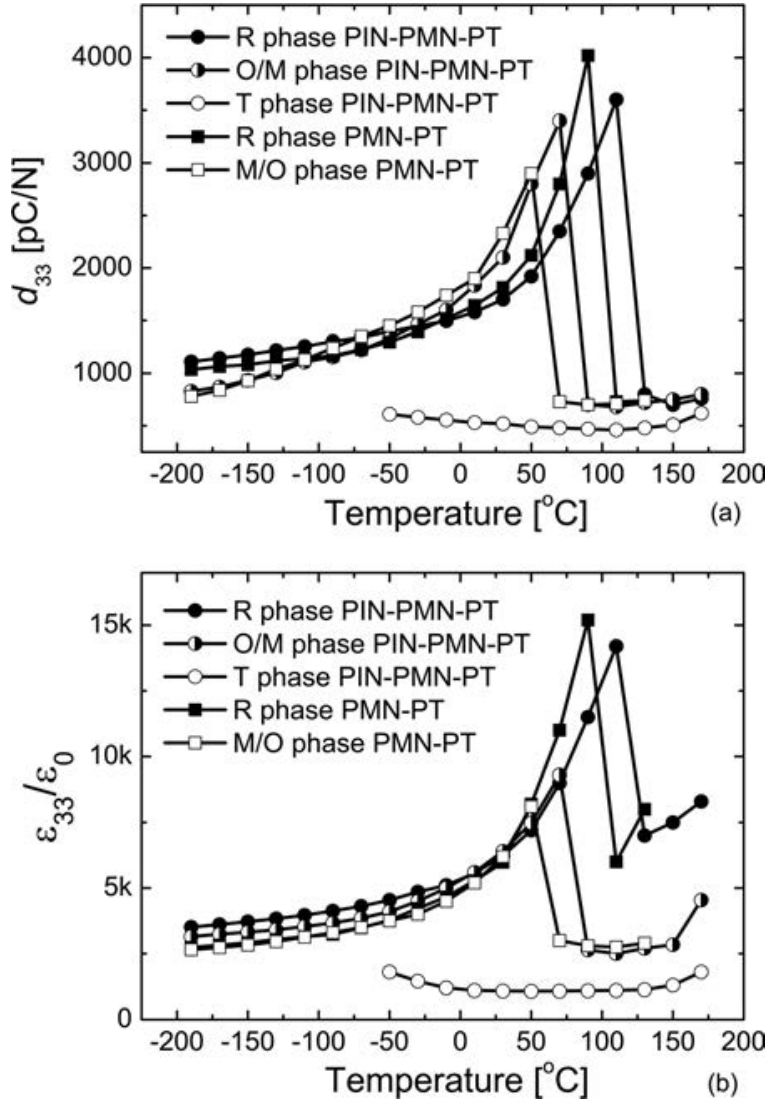

FIG. 27. Temperature dependence of (a) piezoelectric coefficients and (b) relative dielectric permittivities for [001] poled $\mathrm{R}, \mathrm{T}$, and $\mathrm{M} / \mathrm{O}$ crystals.

The spontaneous polarization rotates from $[011]_{\mathrm{C}}$ to $[001]_{\mathrm{C}}$ under an applied field $\mathrm{E}_{1}$ along $[0 \overline{1} 1]_{\mathrm{C}}$ direction, inducing a phase transition from $\mathrm{mm} 2$ to $4 \mathrm{~mm}$ symmetry, which is expected to increase with increasing temperature (approaching the O-T PPT). Of particular significance is that the shear $d_{24}$, with spontaneous polarization rotating from $[011]_{\mathrm{C}}$ to $[111]_{\mathrm{C}}$ under an applied field $\mathrm{E}_{2}$ along $[100]_{\mathrm{C}}$ direction, is closely related to the O-R phase boundary, showing nearly temperature independent behavior (as shown in Figure 28(b)), due to the vertical O-R phase boundary. ${ }^{37,38}$

From the anisotropic calculation given in Figure 11, it is known that the coefficient $d_{33}^{*}$ of [001] poled R and O crystals is mainly attributed to the single domain shear coefficient $d_{15}$. Therefore, the variation of longitudinal piezoelectric coefficients $d_{33}^{*}$ for [001] poled $\mathrm{R}$ and $\mathrm{O}$ crystals shows the same trend of single domain shear coefficient $d_{15}$, as given in Figures 27 and 28. For [001] poled tetragonal crystals, the longitudinal piezoelectric coefficient is stable with respect to temperature, due to the fact that no contribution comes from the shear coefficient $d_{15}$ (no polarization rotation involved), as shown in Figure 27. Of particular importance is that the electromechanical coupling factors of relaxor-PT crystals are found to be insensitive with temperature, as shown in Figure 30 , due to the fact that both dielectric and piezoelectric variations are similar. The coupling factor $k_{33}$ of [001] poled $\mathrm{R}$ and $\mathrm{O}$ crystals, being about 0.90 , slightly increases as the temperature increases up to $\mathrm{T}_{\mathrm{FF}}$, while for [001] poled $\mathrm{T}$ crystals, the coupling factor $k_{33}$ was found to decrease with increasing temperature.
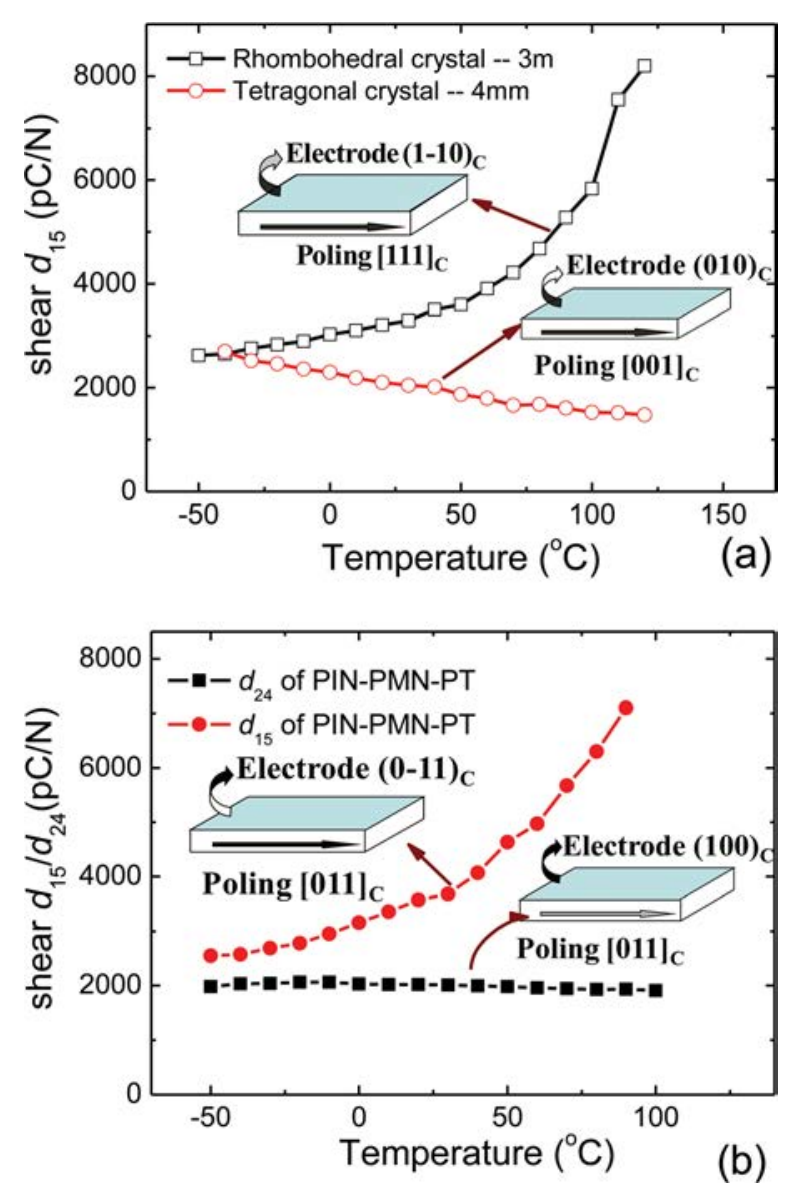

FIG. 28. (Color online) Temperature dependence of single domain shear piezoelectric responses for (a) PIN-PMN-PT crystals with $\mathrm{R}$ and $\mathrm{T}$ phases and (b) PIN-PMN-PT crystals with $\mathrm{O}$ phase. Reprinted with permission from F. Li et al., Applied Physics Letters 97, 252903 (2010). Copyright (c) 2010, the American Institute of Physics.

To fully understand the temperature dependent properties for domain engineered relaxor-PT crystals, the [011] poled tetragonal (with "2 T" engineered domain) and [111] poled orthorhombic (with "3O" engineered domain) crystals were

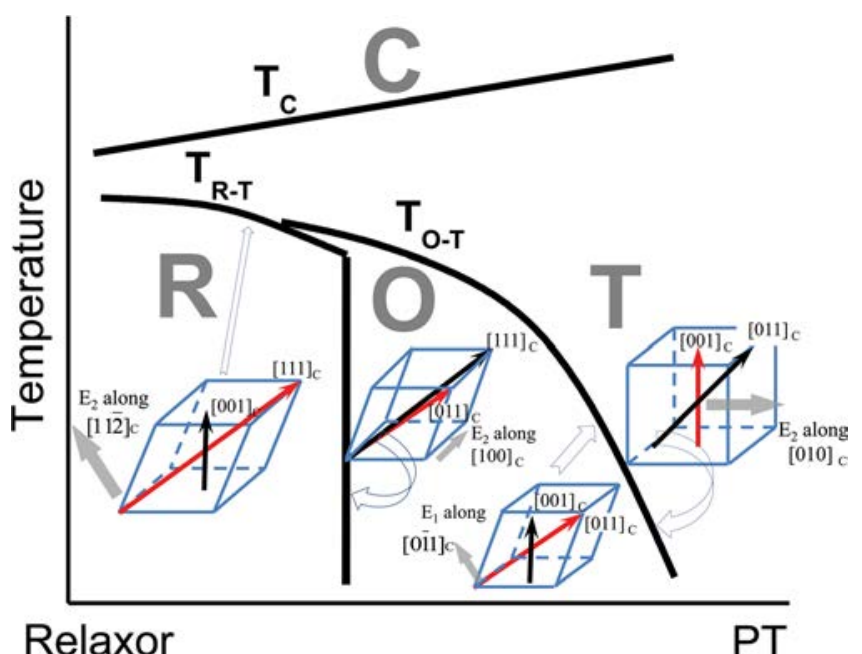

FIG. 29. (Color online) Schematic phase diagram and the polarization rotations for relaxor-PT based crystals. 

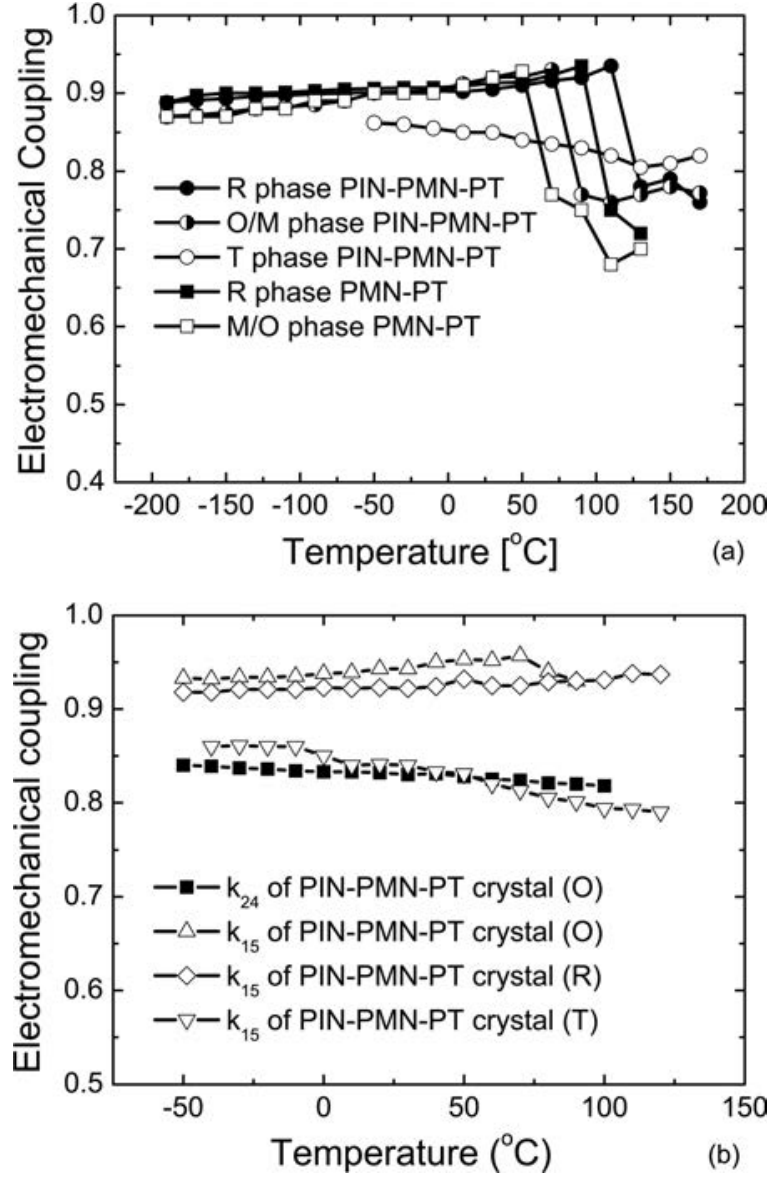

FIG. 30. Temperature dependence of electromechanical coupling factors for relaxor-PT crystals (a) longitudinal mode and (b) shear mode.F. Li et al., Advanced Functional Materials 21, 2118 (2011). (Copyright () 2011, Wiley)

investigated, with the results shown in Figure 31. At room temperature, $d_{33}$ values are around $1000 \mathrm{pC} / \mathrm{N}$ and $800 \mathrm{pC} / \mathrm{N}$ for [011] poled tetragonal and [111] poled orthorhombic relaxor-PT crystals, respectively. From the anisotropic piezoelectric behavior, the longitudinal piezoelectric coefficients for crystals with " $2 \mathrm{~T}$ " and " $3 \mathrm{O}$ " engineered domain configurations are mainly contributed by the single domain shear coefficient $d_{15}$ ("1 T") and $d_{24}$ ("1O"), respectively. It was observed that the $d_{33}$ of [011] poled tetragonal crystal decreases with increasing temperature, following the same trend of the shear coefficient $d_{15}$ in " $1 \mathrm{~T}$ " state (Figure 28(a)). Of particular significance is that for [111] poled O crystals, the coefficient $d_{33}$ is almost independent of temperature $\left(820 \sim 860 \mathrm{pC} / \mathrm{N}\right.$ in the temperature range $20^{\circ} \mathrm{C} \sim 100^{\circ} \mathrm{C}$ ), due to the temperature independent shear $d_{24}$, as shown in Figure 28(b).

Analogous to the piezoelectric and dielectric responses, the losses of relaxor-PT crystals exhibit a strong temperature dependence, as shown in Figure 32, where the mechanical loss was found to increase with increasing temperature, due to increased domain wall motion at elevated temperatures. It should be noted that the dielectric loss also exhibited a similar trend to that of the mechanical loss, which drastically increased as the temperature approached $\mathrm{T}_{\mathrm{FF}} / \mathrm{T}_{\mathrm{C}}$.

Before finishing this section, the properties of relaxorPT crystals at cryogenic temperatures are discussed briefly.
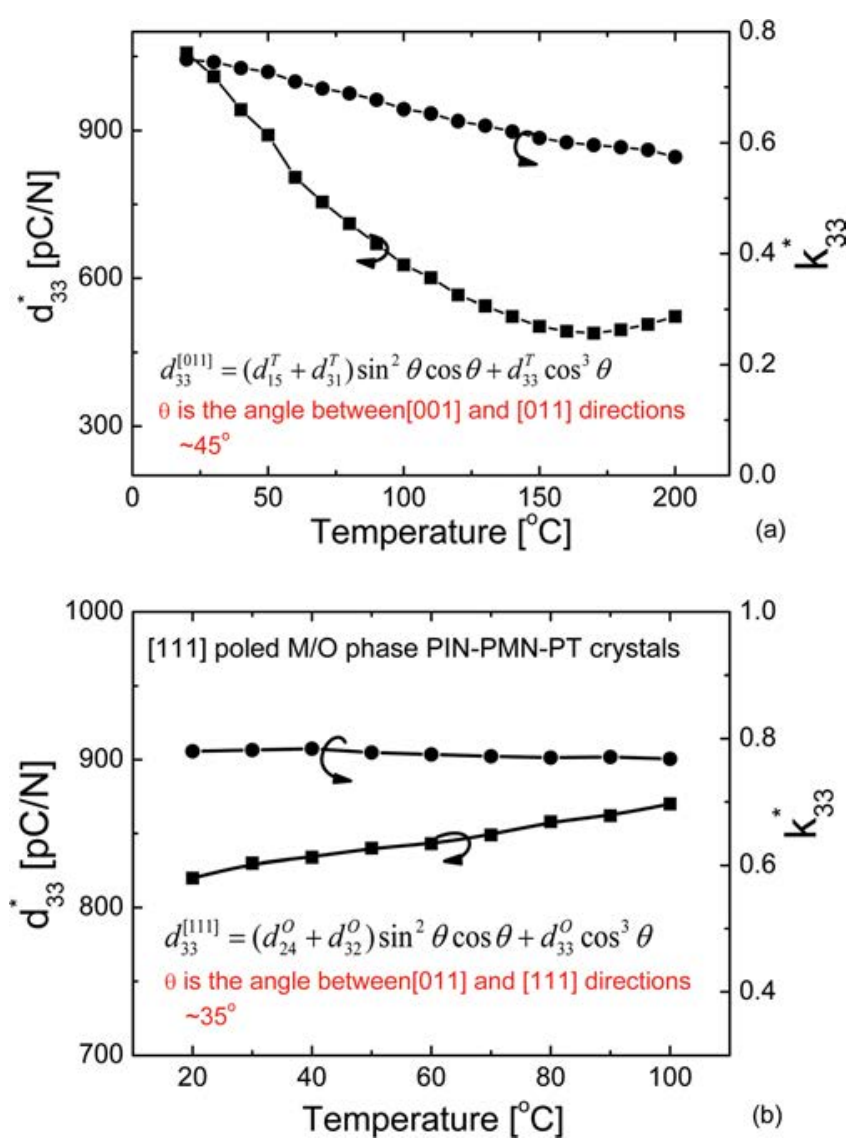

FIG. 31. (Color online) Temperature dependence of longitudinal piezoelectric coefficients and electromechanical coupling factors for (a) [011] poled tetragonal crystals and (b) [111] poled orthorhombic crystals.

At $-190{ }^{\circ} \mathrm{C}$, the longitudinal piezoelectric coefficients of domain engineered relaxor-PT crystals were reported to be $\sim 1000 \mathrm{pC} / \mathrm{N}$, a reduction of $\sim 40 \%$ when compared to room temperature values. For soft PZT and PMN-PT polycrystalline ceramics, however, the piezoelectric coefficients are only on the order of $200 \mathrm{pC} / \mathrm{N}$, a reduction of $60 \%-75 \%$ of the room temperature values. The reduction is much higher than those of relaxor-PT crystals, due to the fact that the

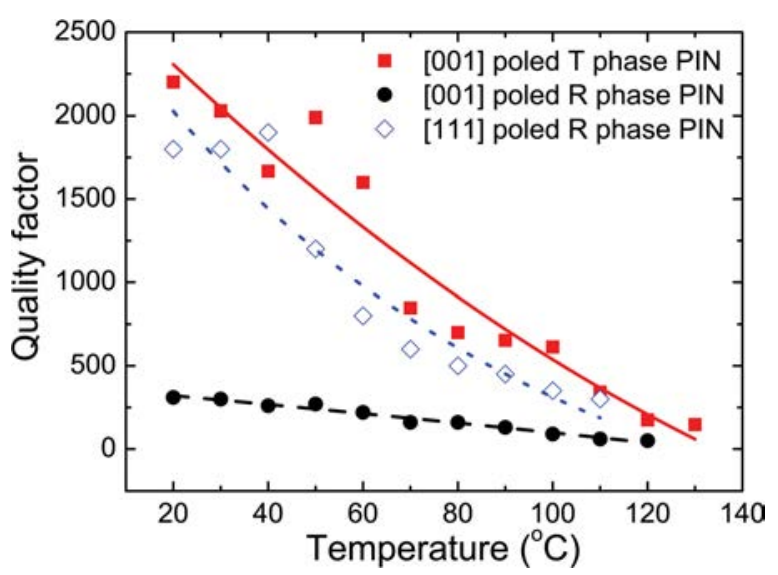

FIG. 32. (Color online) Temperature dependence of mechanical quality factors for PIN-PMN-PT crystals. 


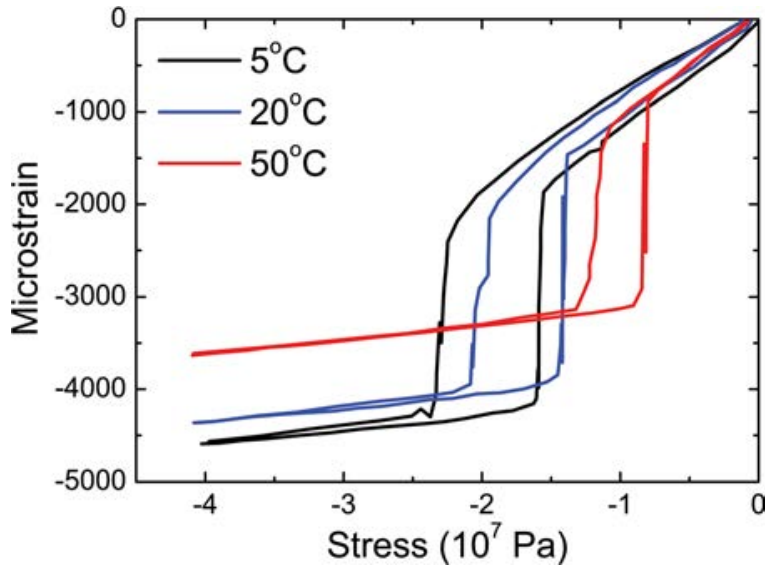

FIG. 33. (Color online) Uniaxial compressive strain-stress response of [001] poled PIN-PMN-PT single crystal at various temperatures and zero dc bias. Reprinted with permission from P. Finkel et al., Applied Physics Letters 97, 122903 (2010). Copyright (C) 2010, the American Institute of Physics.

extrinsic contribution (domain wall motion) is almost fully frozen upon $-150{ }^{\circ} \mathrm{C}$ for polycrystalline ceramics. ${ }^{297}$

\section{B. Uniaxial stress and $d c$ bias field effects}

For transducer applications, a piezoelectric element may be subjected to uniaxial stress and/or $d c$ bias electric fields. ${ }^{25}$ A compressive stress is generally used to prevent the piezoelectric elements from going into tension under high drive field, whereas a $d c$ bias electric field may be applied to stabilize the piezoelectric material and prevent it from depoling. ${ }^{301,302}$ In this section, the effect of compressive stress and positive $d c$ bias field on [001] poled relaxor-PT crystals is reviewed, including stress/electric field induced phase transitions and stress/electric field dependence of piezoelectric properties. ${ }^{33-35,70,75,118,119,287,301-312}$

\section{Stress/electric field induced phase transitions}

The strain versus stress response for [001] poled single crystals has been extensively investigated by Finkel et al., 118 as shown in Figure 33. With the application of a compressive stress, the polarization of [001] oriented rhombohedral crystals rotates from [111] to [011], as analyzed using Devonshire theory, ${ }^{303}$ where the rotation process can be separated into two stages, as shown in Figure 34. Stages I and II correspond to reversible polarization rotation and the $\mathrm{R} \rightarrow \mathrm{O}$ phase transition, respectively, which can be observed in the measurements (Figure 33). The stress induced $\mathrm{R} \rightarrow \mathrm{O}$ phase transition can be recovered upon removal of the stress, and the

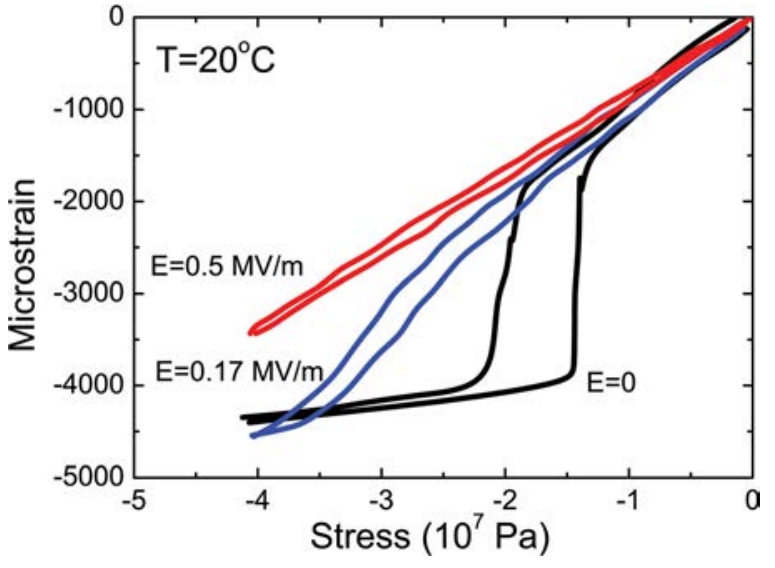

FIG. 35. (Color online) Strain-stress behaviors for relaxor-PT based crystals under various dc bias fields. Reprinted with permission from P. Finkel et al., Applied Physics Letters 97, 122903 (2010). Copyright (C) 2010, the American Institute of Physics.

phase transition stress level is found to decrease with increasing temperature, as shown in Figure 33. From Figures 34 and 16, it can be concluded that the effect of compressive stress and positive $d c$ bias field on polarization rotation is opposite to one another, where a $d c$ electric field was observed to enhance the magnitude of phase transition stress, as shown in Figure $35 .{ }^{118}$ In addition, the hysteresis was found to decrease and the strain-stress response became linear with increasing $d c$ bias electric field, demonstrating that a $d c$ bias field can be used to improve the stability of the relaxor-PT crystals based transducers.

Figure 36 shows the electric field-strain response of [001] poled PMN-PT crystals. ${ }^{116}$ Under an [001] electric field, the polarization rotation is shown in Figure 16, where the polar vectors rotate from [111] to [001] direction. As shown in Figure 36, two phase transitions were observed for PMN0.28PT and PMN-0.30PT crystals, being R-Mc and Mc-T, respectively, which were confirmed by in situ observations. ${ }^{287}$ For $\mathrm{M}_{\mathrm{C}} / \mathrm{O}$ crystals, only one phase transition Mc-T was observed, with the shape of the strain-electric field loops similar to that of strain-stress loops. Analogous to the phase transition stress, the phase transition electric field was found to decrease with increasing temperature, while increasing as the composition moves away from the R-O/Mc phase boundary. ${ }^{33}$ The electric field induced phase transitions in PMN-PT crystals are generally recoverable. However, it should be noted that the phase transition is irrecoverable for relaxor-PT crystals with compositions extremely close to MPBs, ${ }^{305-308}$ where the piezoelectricity degrades drastically after the occurrence of the irrecoverable phase transition.
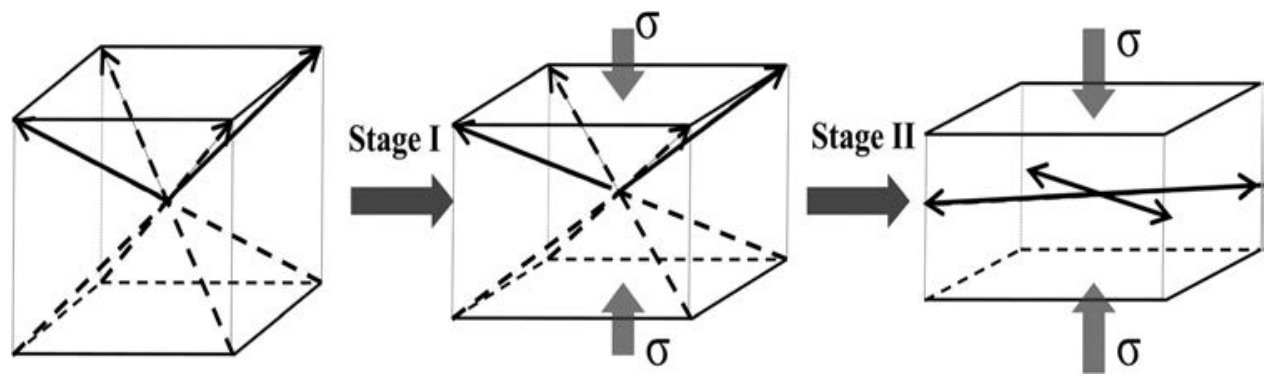

FIG. 34. Polarization rotation and phase transition of [001] poled rhombohedral crystals under uniaxial stress. 


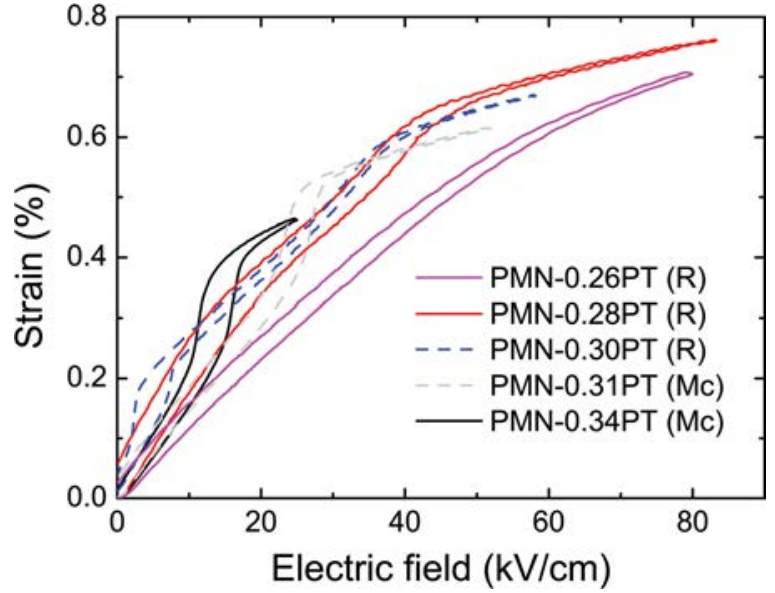

FIG. 36. (Color online) Electric field induced strain for PMN-xPT crystals. (data from Ref. 116).

\section{Piezoelectric properties as a function of $\mathrm{dc}$ bias and uniaxial stress}

a. dc bias field. With the application of a $d c$ bias field, both the intrinsic (lattice) and extrinsic (domain wall motion) contributions to the piezoelectric response in ferroelectrics are affected. In "soft" piezoelectric ceramics, where the contribution of domain wall motion to piezoelectricity is high, being $\geq 40 \%,{ }^{311}$ thus, a clamping effect of domain wall motion is thought to be the dominant factor under $d c$ electric field. ${ }^{312}$ For "hard" piezoelectric ceramics, the piezoelectric response is nearly independent of the positive $d c$ bias field, since domains have been clamped by the internal bias field. The ultrahigh piezoelectric properties of domain engineered relaxor-PT crystals, however, are primarily induced by the lattice deformation, thus, the $d c$ bias effect on relaxor-PT crystals can be analyzed based on the intrinsic contribution.

Figure 37 shows the piezoelectric coefficient $d_{33}{ }^{*}$ for [001] poled PIN-PMN-PT crystals as a function of positive $d c$ bias field. ${ }^{313}$ For $\mathrm{R}$ crystals with low PT content, the phase transition field is on the order of $\sim 50 \mathrm{kV} / \mathrm{cm}, d_{33}{ }^{*}$ was found to monotonously decrease with increasing $d c$ bias field, while for $\mathrm{R}$ crystals in proximity to the MPB with phase transition field of $\sim 25 \mathrm{kV} / \mathrm{cm}$, a slight increase was observed at bias field from 12 to $15 \mathrm{kV} / \mathrm{cm}$. Clearly, an increase of $d_{33}{ }^{*}$ was observed for $\mathrm{O}$ crystals. To explain the experimental results, the applied $d c$ bias field is separated into two portions: $\mathrm{E}_{\|}$and $\mathrm{E}_{\perp}$, where the electric field $\mathrm{E}_{\|}$is along the polar direction, while $\mathrm{E}_{\perp}$ is perpendicular to the polar direction. From thermodynamic analysis, it is known that the electric field $\mathrm{E}_{\|}$decreases the piezoelectric coefficient $d_{15}$ of single domain crystals (mechanism I), while $\mathrm{E}_{\perp}$ leads to the improvement of piezoelectric $d_{15}$ (mechanism II). The high single domain $d_{15}$ corresponds to high longitudinal piezoelectric response in domain engineered crystals. For [001] poled PIN-PMN-PT crystals, both mechanisms I and II affect the longitudinal piezoelectric response.

b. Uniaxial stress. The piezoelectric coefficient $d_{33}{ }^{*}$ and electromechanical coupling factor $k_{33}$ as a function of uniaxial stress for [001] poled PMN-PT crystals (R phase)

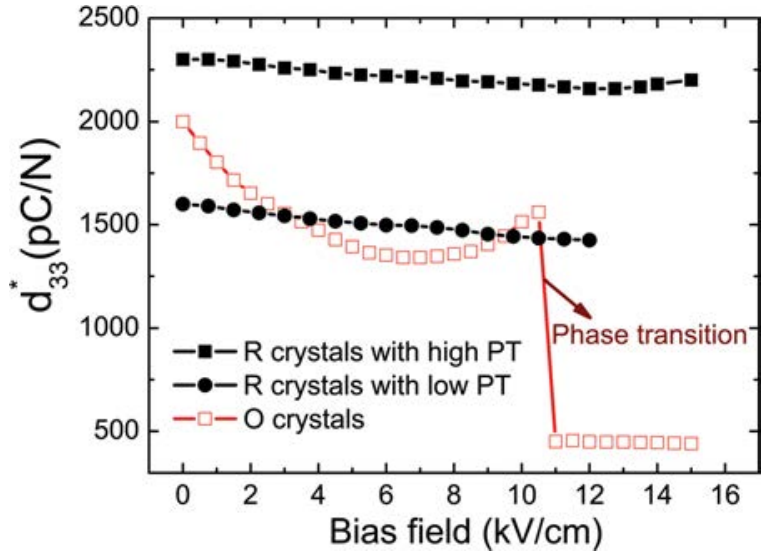

FIG. 37. (Color online) $d c$ bias field dependence of piezoelectric response for PIN-PMN-PT crystals with various phases.

are shown in Figure $38 .{ }^{314}$ The piezoelectric coefficient $d_{33}{ }^{*}$ was found to increase from 1600 to $2500 \mathrm{pC} / \mathrm{N}$ with increasing stress, while the coupling factor showed a slight decrease from 0.95 to 0.90 , which was also reported by Amin et al. ${ }^{301-304}$ The increase in piezoelectric coefficient under compressive stress can be attributed to the stress induced phase boundary motion. As shown in Figure 39, the R-O phase boundary moves to a lower PT region, as a consequence, the rhombohedral crystals approach the R-O phase boundary under stress and give rise to improved piezoelectric properties. ${ }^{302}$ From this respect, therefore, the mechanisms of temperature and stress induced piezoelectric enhancements are similar. Figure 40 shows the strain behaviors for [001] poled PMN-PT crystals under various levels of uniaxial stress, as reported by Viehland et al. ${ }^{314-317}$ It can be observed that the value of strain increased from $2.7 \times 10^{-3}$ to $\sim 4.2 \times 10^{-3}$ under a stress of $4 \times 10^{7} \mathrm{~N} / \mathrm{m}^{2}$, indicating that the zero point strain of the crystal is shifted (i.e., a contraction) under mechanical load and the strain can be (partially or fully) recovered with an application of electric field. If the applied uniaxial stress is high enough, the piezoelectric response of [001] poled relaxor-PT crystals will decrease, ${ }^{301,318,319}$ owing to the stress induced R-O phase transition and depolarization, as discussed in Sec. V B 1. For an application point of view, one needs to pay attention to

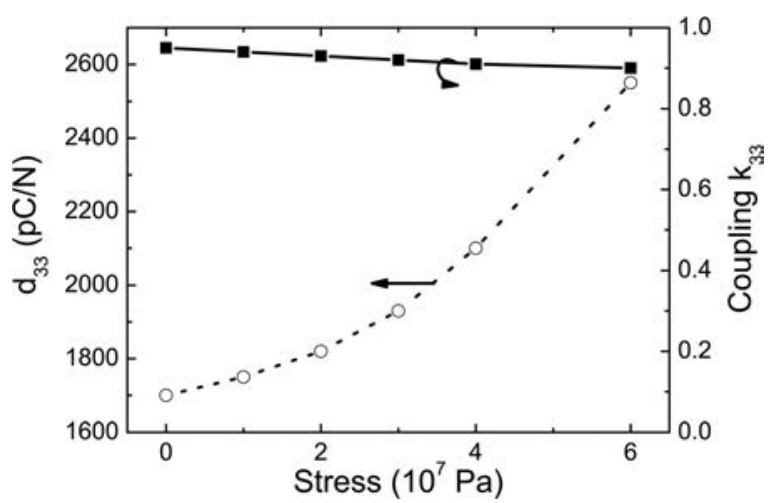

FIG. 38. Uniaxial stress dependence of piezoelectric response for PMN-PT crystals. Reprinted with permission from D. Viehland et al., Journal of Applied Physics 90, 2479 (2001). Copyright (C) 2001, the American Institute of Physics. 


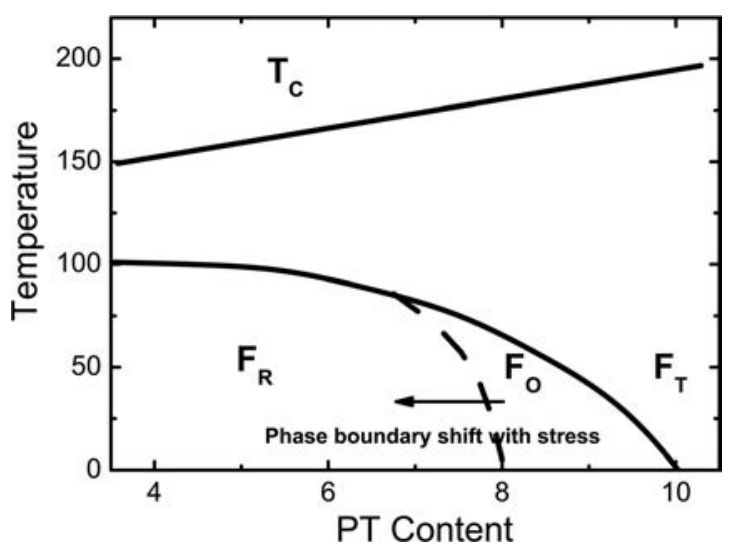

FIG. 39. Schematic for stress induced phase boundary motion for PZN-PT crystals. Reprinted with permission from A. Amin et al., IEEE Transactions on Ultrasonics Ferroelectrectrics Frequency Control 54, 1090 (2007). Copyright $(C)$ 2007, IEEE.

the piezoelectric hysteresis. As shown in Figure 40, the hysteresis of strain-electric field loop increased significantly under compressive stresses.

c. Temperature usage range under dc bias. For relaxor-PT crystals with $\mathrm{R}$ or $\mathrm{M} / \mathrm{O}$ phase, the usage temperature range is limited by their ferroelectric phase transition temperatures, due to the strongly curved MPB. From $d c$ bias experimental results, the usage temperature range for $\mathrm{R}$ or $\mathrm{M} / \mathrm{O}$ crystals is further reduced. ${ }^{320}$ Figure 41 shows a modified phase diagram of the [001] oriented PMN-PT crystals as a function of dc bias, where one can see that the Curie temperature of PMN-PT crystals increases with increasing external $d c$ bias field, while the ferroelectric phase transition temperatures (including R-T and O-T) decreases. This is due to the fact that the applied $d c$ bias lies along [001] orientation, which is the spontaneous polarization direction of $\mathrm{T}$ crystals, thus, the $d c$ bias field stabilizes the tetragonal phase and leads to a decrease in the R/O-T ferroelectric phase transition temperature.

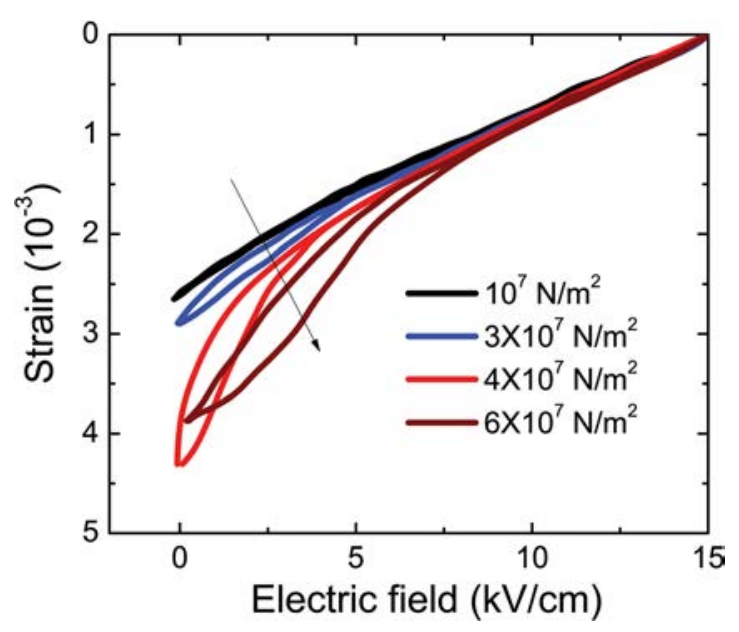

FIG. 40. (Color online) Strain-electric field behaviors for PMN-PT crystals under various prestress. Reprinted with permission from D. Viehland et al., J. Appl. Phys. 90, 2479 (2001). Copyright 2001, the American Institute of Physics.
In this section, the temperature, $d c$ bias field, and uniaxial stress dependent piezoelectric behavior for relaxor-PT based crystals were reviewed. A common characteristic of relaxor-PT crystals under external fields can be established: relaxor-PT crystals possess higher piezoelectric response for compositions close to an MPB, but higher electric/stress field stability and usage temperature range are observed for compositions far away from an MPB. Therefore, based on piezoelectric and related field stability, a compromise should be made in selection of the relaxor-PT crystals for electromechanical applications.

\section{Hydrostatic pressure}

In practical applications, the ferroelectric materials are not only subject to thermal, preload stress, $d c$ bias, and $a c$ drive field, also to hydrostatic pressure condition. Under hydrostatic pressure, the mechanical losses of relaxor-PT crystals were found to significantly increase, due to the enhanced friction between the piezoelectric crystals and pressure transmitting liquid. ${ }^{126}$ High levels of mechanical loss will deteriorate the resonance-antiresonance behavior of the piezoelectric materials, making it difficult to determine the piezoelectric coefficients using the impedance method. Thus, the investigations of piezoelectric properties for relaxor-PT crystals under hydrostatic pressure have been limited. Generally, the variation of piezoelectric coefficients and electromechanical coupling factors for relaxor-PT crystals is small under hydrostatic pressure, up to $500 \mathrm{MPa}$. The piezoelectric $d_{33}$ was reported to decrease from 2050 to $1850 \mathrm{pC} / \mathrm{N}$ for PIN-PMN-PT, while the variation of $k_{33}$ was $\sim 1 \%$ for PMN-PT, ${ }^{126,321-323}$ which can be analyzed with respect to the pressure-induced MPB shifting and/or pressure-induced domain size variation. ${ }^{323}$

\section{Relaxor-PT crystals under high drive field}

\section{Dielectric and piezoelectric properties under high drive field}

Ferroelectrics generally exhibit nonlinear properties under high drive field, owing to the interfaces (domain wall and/or phase boundary) motion. As reported, the piezoelectric and dielectric responses of relaxor-PT crystals generally increase with increasing drive field (electric field or stress). Figure 42 shows the piezoelectric/dielectric responses with respect to the drive field amplitude for relaxor-PT crystals. ${ }^{116,324,325}$ At low levels of drive field (drive field lower than half of the coercive field or stress amplitude lower than $1 \mathrm{MPa}$ ), the dielectric and piezoelectric responses of relaxorPT crystals were found to obey the Rayleigh law, ${ }^{36}$ which gives the approximation of the field dependence of dielectric/piezoelectric coefficient by a linear behavior

$$
m(F)=m_{\text {init }}+\alpha_{m} F,
$$

where the coefficient $m$ refers to the piezoelectric $d$ or dielectric $\varepsilon_{\mathrm{r}}, F$ is the amplitude of drive field (electric field $E$ or stress field $\sigma), m_{\text {init }}$, the zero-field coefficient, and $\alpha$, the Rayleigh coefficient. The coefficient $\alpha$ represents the phase boundary and/or domain wall motion, being related to the 


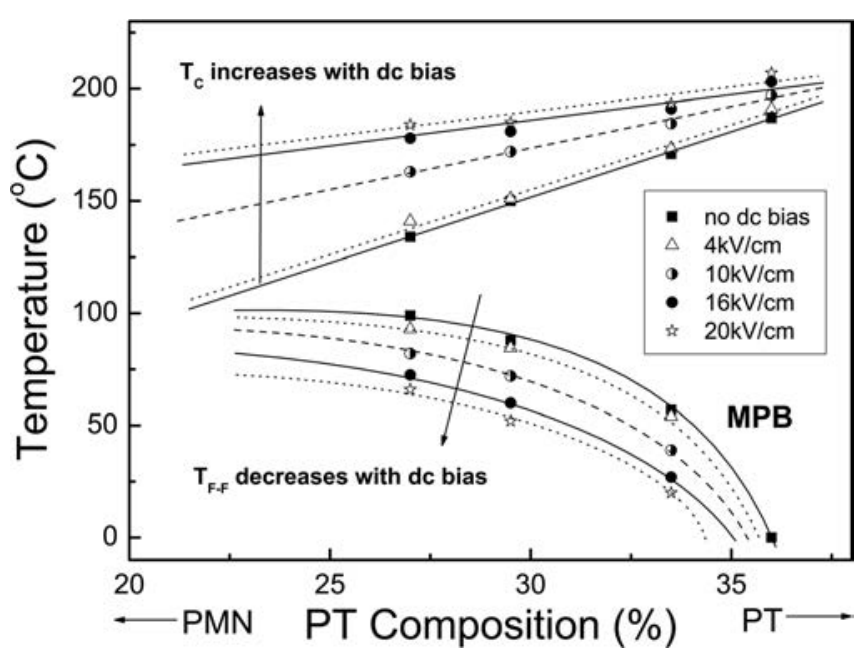

FIG. 41. Phase diagram for PMN-PT single crystals under different $d c$ bias fields. Reprinted with permission from S. J. Zhang et al., in 15th IEEE International Symposium Appl. Ferroeletr. (2006), pp. 261-264. Copyright (C) 2006, IEEE.

composition and orientation of the crystals. As shown in Figure $42(\mathrm{c}),{ }^{116}$ the coefficient $\alpha$ increased as the composition approached MPB, which was discussed in Sec. IV A 6.

\section{Field stability of the shear properties}

The piezoelectric shear properties of single domain relaxor-PT crystals have attracted attention in the last few years, due to their ultrahigh piezoelectric $d_{15}$ and electromechanical coupling $k_{15}$. The ultrahigh elastic compliances make these crystals promising candidates for ultralow frequency transducers. ${ }^{37,38,114,115,243}$ However, the relatively low coercive fields of relaxor-PT crystals, being on the order of $\sim 2-5 \mathrm{kV} / \mathrm{cm}$, limit their applications where large drive fields are required. Table IX lists the principle shear properties for different crystal systems, including piezoelectric coefficients, electromechanical couplings, elastic compliances, frequency constants and coercive fields, etc. The allowable ac drive fields are the maximum applied cyclic electric fields at $10 \mathrm{~Hz}$ frequency, above which, depoling occurs and the shear properties are degraded, while the field stability ratio is the allowable drive field divided by the respective coercive field. ${ }^{253,326}$ The crystal systems investigated include first generation PMN-PT with coercive fields being on the order of $2 \mathrm{kV} / \mathrm{cm}$, second generation PIN-PMN-PT with coercive fields about $4.5-11 \mathrm{kV} / \mathrm{cm}$, and third generation Mn:relaxor-PT with not only higher coercive fields of $6-12 \mathrm{kV} / \mathrm{cm}$ but also internal bias of $0.5-1.5 \mathrm{kV} / \mathrm{cm}$. It was observed that crystals with $\mathrm{T}$ phase possessed lower piezoelectric properties when compared to their $\mathrm{R}$ and $\mathrm{O}$ counterparts, but much higher coercive fields, thus increasing the allowable $a c$ drive field. The field stability ratios of all the unmodified crystals, regardless of the high coercive field in $\mathrm{T}$ crystals, were found to be on the order of $\sim 40 \%$, less than half of their respective coercive fields, whereas the ratios increased to about $60 \%-70 \%$ for third generation crystals, because of the existence of an internal bias field. Thus, the combination of high coercive field and internal bias gives rise to the higher allowable ac drive fields. ${ }^{326}$ Of particular significance is that the face shear mode shows comparable piezoelectric properties to the thickness shear modes, but much higher field stability ratio, being on the same order as the coercive field. ${ }^{253}$ For the face shear case, the working electrode is the same direction as poling direction, where the property degradation above the coercive field is related to the fatigue behavior in [011] poled crystals, rather than the depolarization characteristics observed in thickness shear mode.
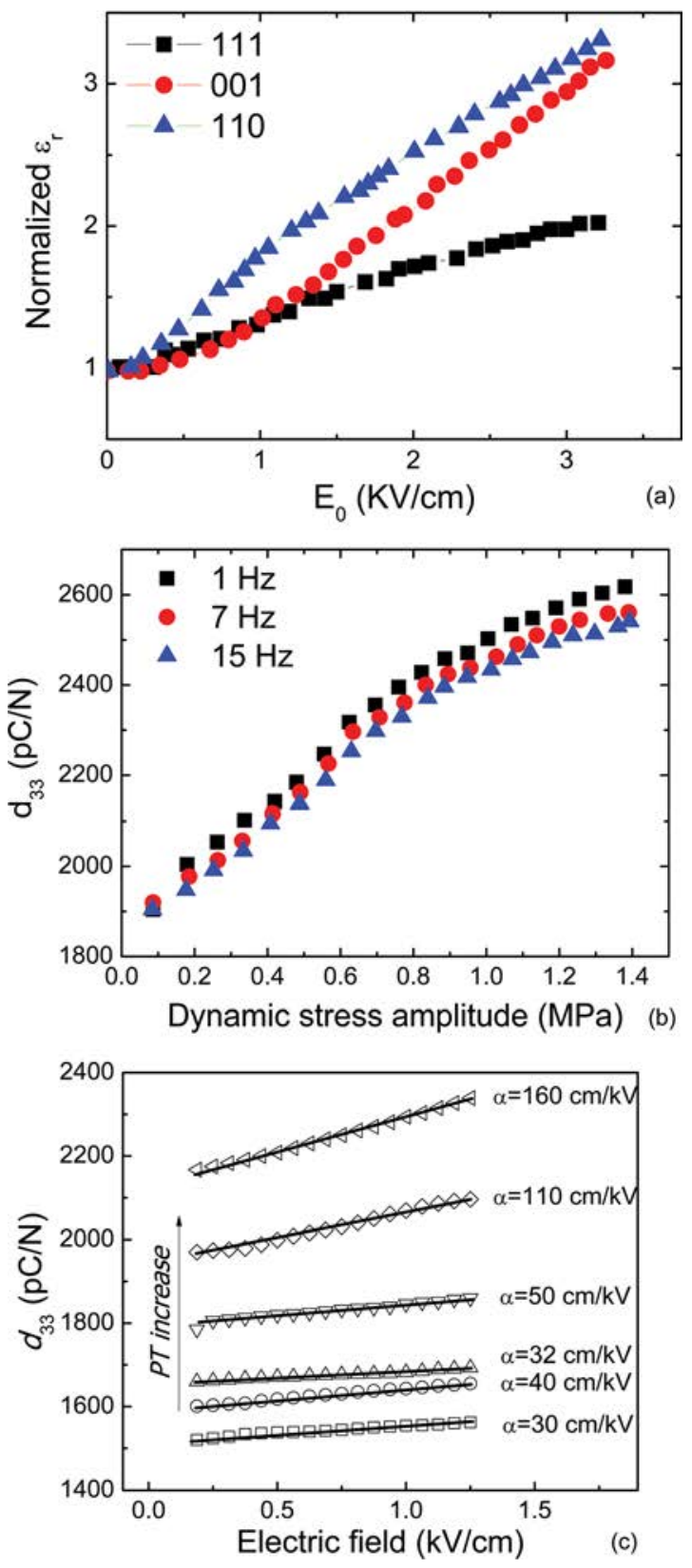

FIG. 42. (Color online) (a) The $a c$ electric field dependent dielectric behavior for PZN-PT crystals, (b) piezoelectric coefficients for PMN-PT crystals as function of dynamic stress, and (c) piezoelectric coefficients for PINPMN-PT crystals as a function of electric field, $\alpha$ values are calculated from the slope of $d_{33}$ vs $E$ curves. Reprinted with permissions from A. Bernal et al., Applied Physics Letters 95, 142911 (2009); M. Davis et al., Journal of Applied Physics 95, 5679 (2004); F. Li et al., Journal of Applied Physics 109, 014108 (2011). Copyright (C 2004, 2009, 2011, the American Institute of Physics. 
TABLE IX. Shear mode characteristics of relaxor-PT crystals with various domain configurations (PIN: PIN-PMN-PT). ${ }^{253,254,326}$

\begin{tabular}{|c|c|c|c|c|c|c|c|c|c|c|}
\hline Poling/Electrode ${ }^{a}$ & $\begin{array}{c}\text { Engineered } \\
\text { domain }\end{array}$ & Crystal & $\mathrm{E}_{\mathrm{C}}(\mathrm{kV} / \mathrm{cm})$ & $\mathrm{E}_{\text {int }}(\mathrm{kV} / \mathrm{cm})$ & $\varepsilon_{\mathrm{r}}$ & $\mathrm{d}_{i j}(\mathrm{pC} / \mathrm{N})$ & $\mathrm{k}_{\mathrm{ij}}$ & $\mathrm{Nr}(\mathrm{Hz} \cdot \mathrm{m})$ & $\begin{array}{l}\text { Allowable ac } \\
\text { field }(\mathrm{kV} / \mathrm{cm})^{\mathrm{b}}\end{array}$ & $\begin{array}{c}\text { Field stability } \\
\text { ratio }\end{array}$ \\
\hline $111 / 1 \overline{1} 0$ & $1 \mathrm{R}\left(\mathrm{d}_{15}\right)$ & PMNT & 2.5 & - & 6000 & 2500 & 0.90 & 500 & $<1$ & $<40 \%$ \\
\hline \multirow[t]{2}{*}{$111 / 1 \overline{1} 0$} & $1 \mathrm{R}\left(\mathrm{d}_{15}\right)$ & Pure PIN & 4.5 & - & 6000 & 3500 & 0.93 & 470 & 2 & $44 \%$ \\
\hline & & PIN-Mn & 6.2 & 1.0 & 8000 & 4100 & 0.94 & 410 & 4 & $65 \%$ \\
\hline \multirow[t]{2}{*}{$011 / 0 \overline{1} 1$} & $2 \mathrm{R}\left(\mathrm{d}_{15}\right)$ & Pure PIN & 5.0 & - & 6500 & 2800 & 0.92 & 570 & 2 & $40 \%$ \\
\hline & & PIN-Mn & 7.3 & 1.2 & 4600 & 2200 & 0.91 & 520 & 5 & $68 \%$ \\
\hline \multirow[t]{2}{*}{$011 / 0 \overline{1} 1$} & $10\left(\mathrm{~d}_{15}\right)$ & Pure PIN & 5.5 & - & 5600 & 3400 & 0.95 & 380 & 2 & $36 \%$ \\
\hline & & PIN-Mn & 9.0 & 0.6 & 5800 & 3500 & 0.95 & 360 & 5.5 & $61 \%$ \\
\hline \multirow[t]{2}{*}{$001 / 100$} & $1 \mathrm{~T}\left(\mathrm{~d}_{15}\right)$ & Pure PIN & 12.0 & - & 15000 & 2200 & 0.85 & 850 & 4.5 & $38 \%$ \\
\hline & & PIN-Mn & 11.5 & 1.5 & 8000 & 1200 & 0.77 & 950 & 7.0 & $61 \%$ \\
\hline $001 / 110$ & $1 \mathrm{~T}\left(\mathrm{~d}_{15}\right)$ & PIN-Mn & 11.7 & 1.5 & 8000 & 1200 & 0.78 & 980 & 8.5 & $72 \%$ \\
\hline 011/011 & $2 \mathrm{R}\left(\mathrm{d}_{36}\right)$ & Pure PIN & 5.0 & - & 5000 & 2600 & 0.82 & 500 & 5.0 & $100 \%$ \\
\hline
\end{tabular}

${ }^{\mathrm{a}}$ First [hkl] is poling direction and second (hkl) is electrode face.

${ }^{\mathrm{b}}$ Allowable ac fields achieved here are at $10 \mathrm{~Hz}$ ac drive field condition, for practical applications, higher allowable drive fields are expected at higher drive field frequency.

It should be noted that in the pulse-echo ultrasound imaging systems, a transducer is excited by short duration electrical pulses. The depolarization behavior of relaxor-PT single crystals in thickness extensional mode has been studied using electrical pulses with $10 \mathrm{~ns}$ duration and $\ll 1 \%$ duty cycle, showing high field stability, with the allowable pulse field about three times of the coercive field, regardless the different coercive field values for various crystal systems.

\section{Fatigue behavior}

The fatigue behavior of binary PMN-PT, PZN-PT crystals, and ternary PIN-PMN-PT crystals with various crystallographic directions have been investigated. ${ }^{327-337}$ Figure 43 shows the polarization as a function of bipolar cycles for relaxor-PT crystals. ${ }^{330-332}$ The polarization was found to decrease with increasing bipolar cycles for [011] and [111] oriented crystals, exhibiting fatigue behavior, while [001] oriented crystals shows fatigue-free characteristics, revealing anisotropic fatigue behavior. The degradation of polarization (fatigue) for relaxor-PT crystals was mainly attributed to the effect of charge-injection and micro/macro-cracks. Chargeinjection and accumulation of space charge that pins domain wall movement is a generally agreed upon mechanism for fatigue in ferroelectrics. ${ }^{327,328}$ Lee et al. ${ }^{330}$ proposed that the effect of charge-injection was much more significant for [111] oriented crystals than crystals oriented along [001] and [011] directions, due to the fact that the sideways growth of domains and lower density of domain boundaries in [111] oriented $\mathrm{R}$ crystals will increase the concentration of defects, segregating at the domain boundaries and leading to the significant fatigue in the [111] oriented PZN-PT crystals. Due to the injected charge, an internal bias was observed for [111] oriented PZN-PT crystals after fatigue measurements, which induced the islands of frozen domains, resulting in asymmetric strain-field loops. ${ }^{328,329}$ Of particular interest is that the magnitude of the (switchable) polarization and symmetry in hysteresis loops can be rejuvenated for [111] oriented fatigue samples by annealing the crystals above their respective Curie temperatures $\left(\sim 300^{\circ} \mathrm{C}\right)$, leading to redistribution and/or reduction of defects.

For [011] oriented relaxor-PT crystals, a sudden reduction of polarization was observed after the first hundred cycles, following a logarithmic behavior. Based on the analysis by Zhang et al., ${ }^{332}$ the sudden reduction of polarization was attributed to micro/macro cracks, which are in the range of a few tens to a few hundred microns, as observed by scanning electronic microscopy. The cracking in [011] oriented samples was due to the incompatible strain around the non$180^{\circ}$ domain boundaries and large anisotropic piezoelectric strain/displacement, ${ }^{332}$ associated with the $\mathrm{mm} 2$ macroscopic symmetry. Different from the fatigue characteristics observed in [111] oriented crystals, the fatigue behavior in [011] crystals is due to mechanical failure (cracking) and phase transition, which is not recoverable. ${ }^{332}$ The [001] oriented crystals generally show fatigue resistance as compared to those [111] and [011] oriented crystals. However, it should be noted that fatigue behavior was sometimes observed in [001] oriented PMN-PT crystals as well, being attributed to microcracks developed under cyclic electric field, which pin the domain wall motion, as observed for [001] oriented PMN-PT crystals with an MPB composition and large domain size $(>10 \mu \mathrm{m}) .^{334-337}$

In summary, the fatigue phenomena in relaxor-PT crystals are generally attributed to effects of charge-injection and micro/macro-cracks during cycling. The fatigue is also related to the amplitude of applied electric field, ${ }^{328-332}$ with the crystals showing improved fatigue behavior when the cyclic electric fields are less than their respective coercive fields. Thus, acceptor doped crystals with an internal bias and relatively higher coercive fields are expected to show improved fatigue-resistant behavior, which was found to be the case for Mn:PIN-PMN-PT crystals. ${ }^{332}$

It should be noted that the high drive field discussed above is at off-resonance frequency. For high power applications, however, the transducers need to be operated near or at the resonance, where the relaxor-PT crystals behave very differently. The dynamic strain and the output power are not 

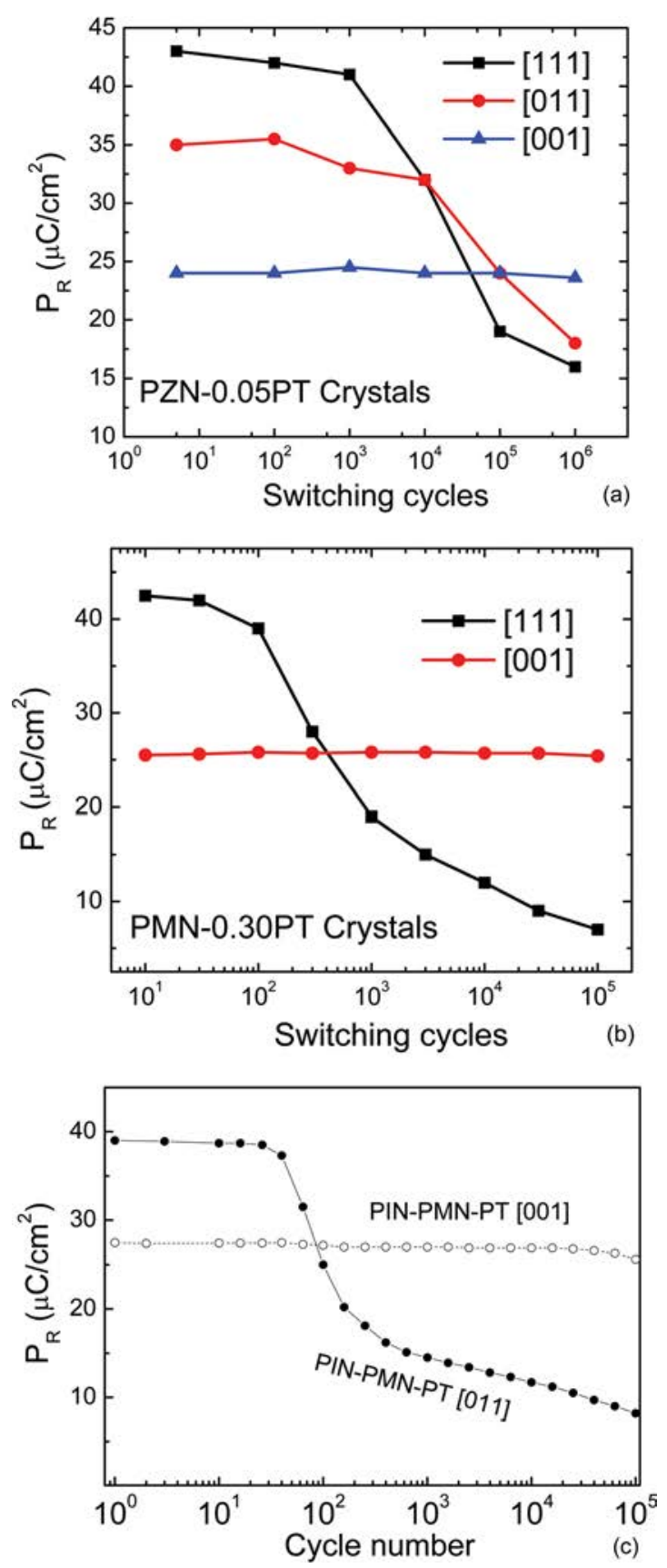

FIG. 43. (Color online) Fatigue behavior for relaxor-PT crystals (a) PZN0.05PT. Reprinted with permission from J. K. Lee et al., Journal of Applied Physics 96, 7471 (2004). Copyright (C) 2004, the American Institute of Physics) (b) PMN-0.30PT. Reprinted with permission from Y. Zhang et al., Journal of European Ceramic Society 24, 2983 (2004). Copyright ( 2004, Elsevier, and (c) PIN-PMN-PT crystals (data from Ref. 332).

only related to the piezoelectric property and applied electric field, but also associated with the mechanical loss. ${ }^{137,294-296}$

\section{APPLICATIONS}

In this section, the potential of relaxor-PT single crystals for various applications, including ultrasound transducers, sensors, and actuators, is evaluated. Advantages and issues of crystals in relation to state-of-art polycrystalline ceramics for electromechanical applications will be discussed. In contrast to polycrystalline piezoelectric ceramics, the ability of domain engineered configurations, crystallographic directions (anisotropic characteristics), and crystal phases offer novel vibration modes and properties not achievable in polycrystalline ceramics.

\section{A. Ultrasound transducers}

Ultrasound transducer is a device that converts electrical energy (for example, alternating current) into ultrasound (mechanical or acoustic energy). For transducer applications, the key material parameter is the electromechanical coupling factor, which is closely related to device bandwidth and sensitivity. In addition, the dielectric permittivity is a critical parameter in order to match the electrical impedance of transducer to that of driving electronics. Temperature and field stabilities are also important for ultrasound transducers, since the dielectric permittivity changes as a function of temperature and field, resulting in the variation of electrical impedance of device. ${ }^{338}$

The most widely studied transducer application for relaxor-PT crystals has been the medical ultrasound, allowing for improved image resolution. ${ }^{186,338-369}$ The application of medical ultrasonic imaging spans a wide frequency range, from 1.5 to $60 \mathrm{MHz}$, depending on the organs to be imaged. For abdominal, obstetric, and cardiological imaging applications, the frequency range is from 2 to $5 \mathrm{MHz}$. For pediatric and peripheral vascular applications, the range is from 5 to $7.5 \mathrm{MHz}$, while the ophthalmology, dermatology, intracardiac, and intravascular imaging require frequencies in the range of 10 to $>60 \mathrm{MHz} .{ }^{339}$ Generally, low mechanical Q of transduction structures is desired for medical imaging transducers, which allows for better sharp pulses detection.

Underwater sonar projector is another type ultrasound transducer, which has been extensively studied. ${ }^{186,294,370-385}$ Different from high frequency and low duty cycle medical ultrasonic transducers, sonar projectors usually operate in a much lower frequency range $(1-500 \mathrm{kHz})$ and higher duty cycle (up to $100 \%$ ). This requires that the piezoelectric materials possess not only high coupling factors but also high coercive fields ( $a c$ field stability), mechanical quality factors (less heat generation) and high elastic compliances (low frequency range operation, and/or device miniaturization). In this section, we will focus on the recent developments on the medical ultrasonic transducer and sonar projectors based on relaxor-PT single crystals.

\section{Medical ultrasonic transducers}

For medical imaging applications, ultrasonic piezoelectric transducers are operated in the thickness mode, where a piezoelectric element is located between acoustic matching layers and backing layer. Piezoelectric materials with high thickness mode coupling, low acoustic impedance, and pure thickness mode operation are required to achieve high sensitivity and broad bandwidth. ${ }^{186,339}$

Piezoelectric material/polymer composites utilizing 1-3 and 2-2 connectivity, offer advantages over single phase piezoelectric materials, ${ }^{12,386-389}$ as shown in Figure 44, with the highest electromechanical coupling observed in 1-3 composites. In addition, the acoustic impedance of composites is 
greatly decreased when compared to monolithic piezoelectric materials, due to the low density epoxy constituent. Furthermore, the properties of composites can be tuned over a wide range by choosing the volume fraction and compositions of each constituent.

The state-of-art piezoelectric ceramics used in medical transducers are generally soft PZTs, e.g., PZT5H, which offer relatively high longitudinal coupling $k_{33} \sim 0.75$ and sliver coupling $k_{33}$ ' $\sim 0.70$ for array transducers. Single crystals offer ultrahigh longitudinal $k_{33}>0.90$ and sliver $k_{33}$ ' $>0.80$, achieved in the [001] direction, which can be utilized in the 1-3 or 2-2 composite structures, respectively. The resulting bandwidths, over $130 \%$, can be used for a variety of applications including sub-harmonic or second harmonic imaging for increased resolution. ${ }^{338-345}$

Several companies, including Philips, GE, Siemens, Hitachi, Toshiba, TRS Technologies, Humanscan, Boston Scientific, HC Materials and etc., have been employing relaxor-PT crystals for medical ultrasonic applications. Toshiba was a pioneer in this field, working on the development of single crystals and phased array transducers for medical imaging applications starting in the $1990 \mathrm{~s}$, while the first low frequency phased array transducer was released to the market in November 2004 by Philips and received excellent market acceptance. ${ }^{338}$ Since then, different generations of medical imaging transducers have been developed, with improvements in bandwidth and sensitivity, being on the order of $>15 \%$ and $>3 \mathrm{~dB}$, respectively, when compared to their PZT ceramic counterparts. In addition to the above advantages, an increase in hydrostatic parameters of crystal/epoxy composites was reported when compared to ceramic/epoxy composites, providing a figure of merit $\left(d_{h} g_{h}\right)$ more than three times that of PZT composites, improving the signal to noise ratio (SNR) and providing either deeper penetration or lower excitation amplitudes. This is particularly important in emerging highfrequency ultrasound imaging applications. ${ }^{186}$

Fabrication of crystal/epoxy composites are generally the dice-and-fill process, as shown in Figure 45. Due to the low fracture toughness and possible high internal stresses within the crystals, single crystal wafers are prone to chipping and crack propagation during the dicing process, thus optimized dicing conditions, including selection of blade type, dicing speed, dicing depth, and supporting material, are required. ${ }^{358-360}$ For composites with an operational frequency range above $20 \mathrm{MHz}$, the post (pillar) widths need to be less than $50 \mu \mathrm{m}$ to keep longitudinal aspect ratio, which is not practical using mechanical dicing. A photolithography based micromachining process was developed by TRS, ${ }^{358}$ based on micromachined ultrasound transducer (PC-MUT) technique, as shown in Figure 46. This PC-MUT has been demonstrated successfully for high frequency imaging applications, possessing the advantages of submicron machining precision, batch fabrication, and a low-stress mechanical environment for fragile, fine structures. ${ }^{358-360}$

The obtained electromechanical coupling factors for PCMUT PMN-PT/epoxy composites were reported to be $\sim 0.79$ for $15 \mathrm{MHz}$ transducer, showing great potential compared to PZT composite. However, the coupling decreased to 0.67 for $75 \mathrm{MHz}$ transducer, exhibited a large decrease in electrome-
1-3 Composite Longitudinal mode $k_{33}>0.9$

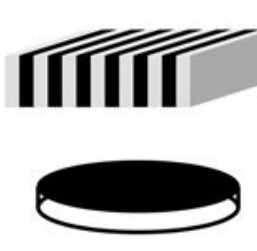

2-2 Composite Sliver mode $\mathrm{k}_{33}{ }^{\prime} \sim 0.8$

Monolithic plate Thickness mode $\mathrm{k}_{\mathrm{t}} \sim 0.6$

FIG. 44. Properties of 1-3 and 2-2 composites, compared to monolithic plate.

chanical coupling, showing a strong scaling effect and leading to the question of the origin for property degradation in PMNPT crystals at high frequencies. ${ }^{390-392}$ Figure 47 shows the electromechanical properties of crystal/epoxy 1-3 composites as a function of sample thickness. ${ }^{390}$ For comparison, the longitudinal coupling factors $k_{33}$ of monolithic PMN-PT and PIN-PMN-PT samples, which were calculated according to Eq. (20), were plotted as a function of sample thickness. In addition, the reported coupling factors for PMN-PT/epoxy 1-3 composites were included. Note that the corresponding resonance frequencies on top $\mathrm{X}$-axis were calculated from the frequency constant by assuming $\sim 1000 \mathrm{~Hz}$ m for both monolithic and 1-3 composites samples. From Figure 47, the monolithic PMN-PT crystals were found to exhibit a decrease in coupling $\left(k_{33}\right)$ with decreasing sample thickness, due to the degradation of free dielectric permittivity, a result of domain size/surface boundary clamping. ${ }^{390,391}$ For crystal/epoxy 1-3 composites, the level of decrease in coupling was found to be greater than that of monolithic samples (larger slope observed), demonstrating that the surface damage induced by

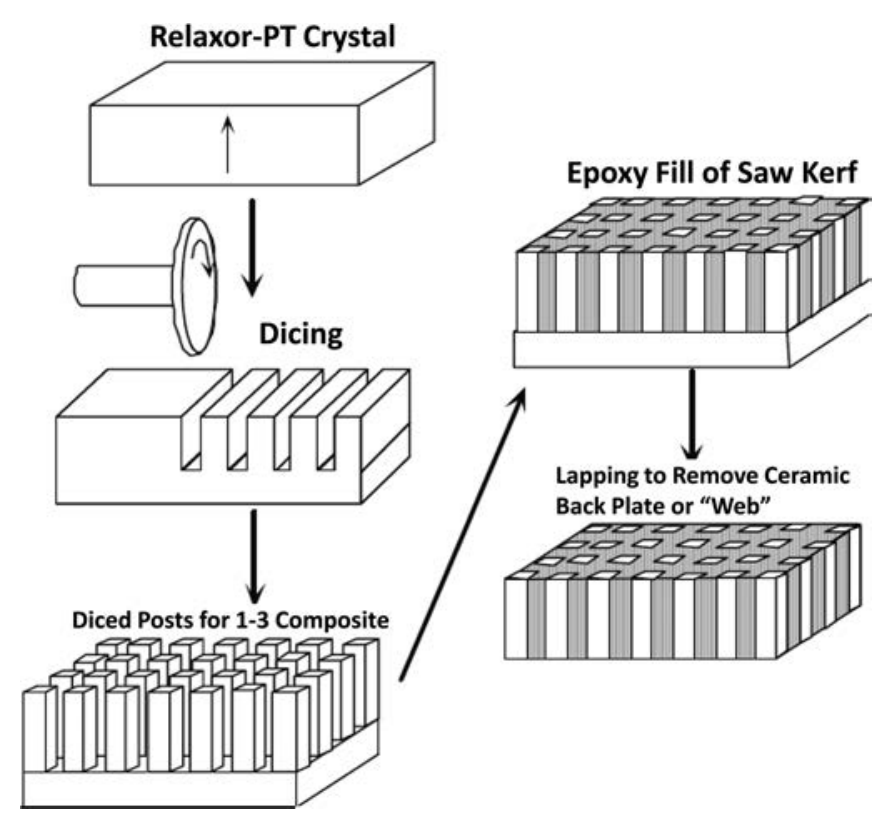

FIG. 45. Composite transducers dice-and-fill process. P. W. Rehrig et al., "Micromachined imaging transducer," U.S. patent US7622853 (24 November 2009); X. N. Jiang et al., Proceedings on SPIE 6531, 65310 F (2007); X. N. Jiang et al., Proceedings on SPIE 6934, 69340D (2008). Copyright (C) 2008, SPIE. 


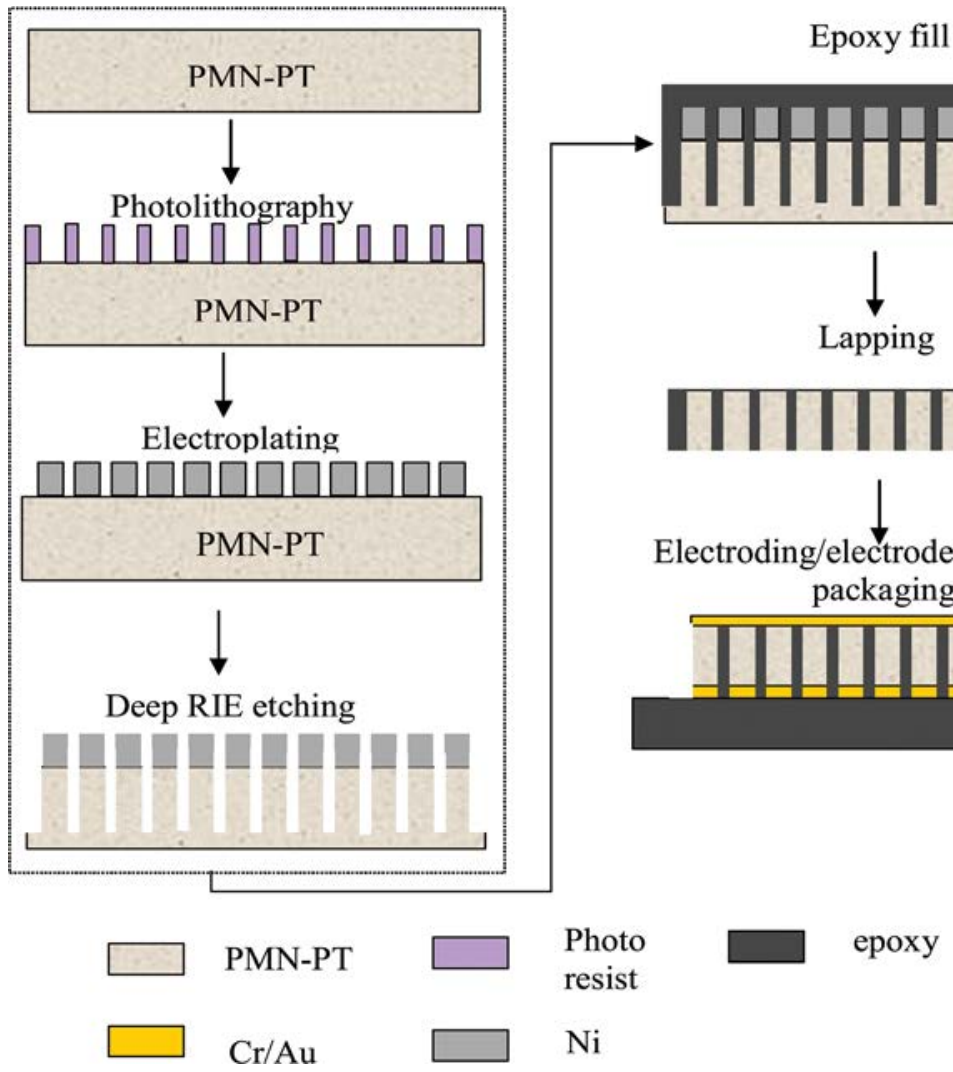

the dicing process, gave rise to further property degradation. ${ }^{391}$ It was observed that the $20 \mathrm{MHz}$ PIN-PMN-PT/epoxy 1-3 composite maintained higher electromechanical coupling factors, being on the order of 0.80 , when compared to $20 \mathrm{MHz}$ PMN-PT composites, with coupling being only 0.74 . This is due to the fact that the domain size of PMN-PT crystals $(10-20 \mu \mathrm{m})$ is much larger than that of PIN-PMN-PT $(\sim 1 \mu \mathrm{m})$. When the physical size of the samples $(\sim 50 \mu \mathrm{m})$ becomes of the same order as the domain size, it is possible that the surface boundary condition may disrupt the equilibrium " $4 \mathrm{R}$ " domain structures and hinder the polarization rotation, leading to the decreased dielectric properties and electromechanical coupling in PMN-PT. ${ }^{390,391}$ In order to confirm the role of domain size on the dielectric and piezoelectric properties in ultrathin samples, a field-cool poling approach was applied to PMN-PT crystals, where significantly smaller domain size $\sim 5 \mu$ m was achieved, consequently, the dielectric constant and piezoelectric coefficients were greatly improved. ${ }^{392}$ Thus, ultrahigh frequency PC-MUT composites with much higher electromechanical coupling factors are expected by using the single crystals with small domain size («sample thickness).

Though not discussed in this review, another important medical transducer area is the high power therapeutic ultrasound or high intensity focused ultrasound for cancer treatments, which may benefit from the high performance crystals, especially the third generation crystals.

\section{Underwater acoustic transducers}

Underwater acoustic transducers are useful in ocean engineering. Networked underwater communication systems involve many acoustic modems, each with a projector and a hydrophone, since the materials used in projectors and hydrophones are very different, we will categorize hydrophones in Sec. IV B titled "sensor," while only underwater projectors will be discussed here. ${ }^{385}$

Sonar projectors require significantly high demanding acoustic performance characteristics (high power and high duty cycle), with operational distance ranging from meters to kilometers and frequency operation of $1-500 \mathrm{kHz}$. In these applications, high acoustic source levels are required. Additionally, the variations in temperature, hydrostatic pressure, and high attenuation of the water, etc., complicate the structure and performance of the devices. ${ }^{186,385}$ For a transducer operating at resonance frequency, the mechanical heat generation can be evaluated following the formula:

$$
P_{m}=\frac{Y_{r} \omega S^{2}}{2 Q_{m}},
$$

where $P_{m}$ is the time averaged power dissipation per unit volume, $Y_{r}$ the short circuit modulus, $Q_{m}$ the mechanical quality factor, $S$ the dynamic strain, and $\omega$ the operating frequency.

Autonomous underwater vehicles (AUVs) have been widely used for underwater oil or mineral mining, cable inspection, oceanographic research, salvage, undersea equipment inspection, and maintenance. There are four areas of design that are important in successful deployment of these devices: power consumption, size, detection capability, and communication. ${ }^{186}$ Flextensional "cymbal," 1-3 composites, and tonpilz have been demonstrated to be effective structures for sonar projectors, where tonpilz arrays are currently the 


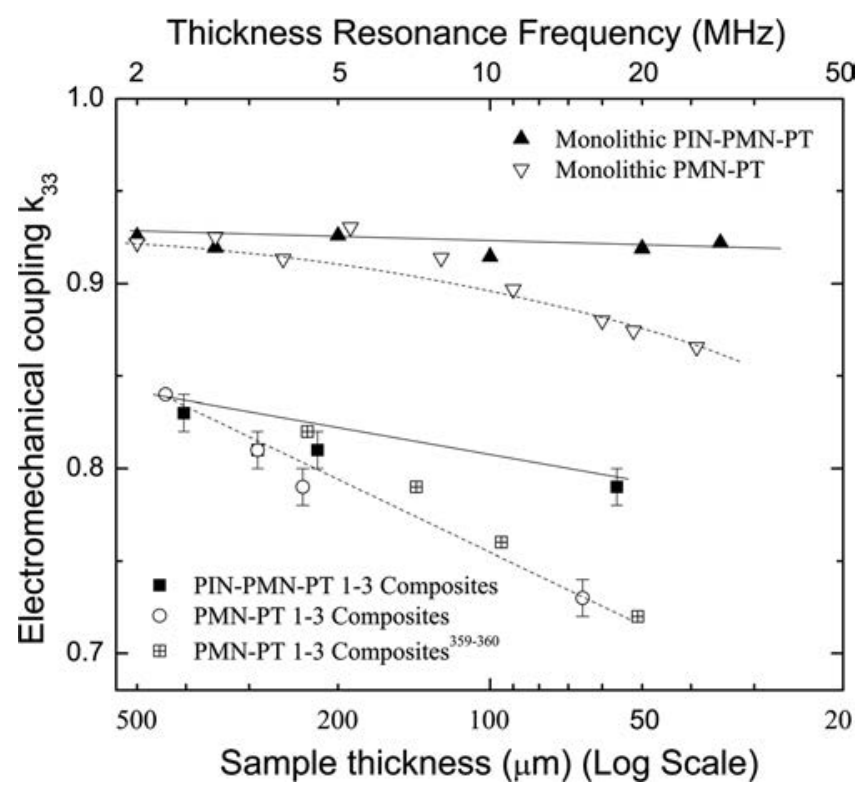

FIG. 47. Electromechanical coupling $k_{33}$ for monolithic and crystal/epoxy 1-3 composites, as a function of sample thickness and corresponding ultrasound frequency. Reprinted with permission from H. J. Lee et al., Journal of Applied Physics 107, 124107 (2010). Copyright (C) 2010, the American Institute of Physics.

most widely studied projectors to obtain the required source level and bandwidth for high power sonar. The start-of-art materials used for sonar projectors include "hard" PZTs, such as PZT4 and PZT8. ${ }^{186,294,373,385}$ Recently, high performance relaxor-PT single crystals have been studied extensively, due to their higher electromechanical coupling and piezoelectric coefficients, when compared to their polycrystalline counterparts. In addition, the Young's modulus is approximately one-quarter that of traditional PZT4 and 8 ceramics, resulting in a significant decrease in device size while maintaining the same frequency. ${ }^{186,295,385}$ Figure 48 gives a schematic figure of a PMN-PT crystal based tonpilz transducer. ${ }^{186,295}$ In tonpilz transducers, the length of the piezoelectric stack is generally larger than the width, which allows the overall stack to operate in a near-longitudinal mode, taking advantage of the high longitudinal coupling $>0.90$ of single crystals. The size of a tonpilz transducer is reduced over $50 \%$ compared to its ceramic counterpart. ${ }^{295} \mathrm{~A}$ sixteen-element array of 33-mode crystal tonpilz transducers was recently reported to provide three times the bandwidth of a comparable PZT ceramic array, producing as much as $15 \mathrm{~dB}$ of additional source level at the band edges with no change in amplifier. ${ }^{302}$

PMN-PT single crystals, however, are "soft" piezoelectric materials and can be depoled under nominal drive fields due to their relatively low coercive fields, thus they require an external $d c$ bias to eliminate depoling. Under bias, PMNPT crystals offer significantly higher energy densities than PZT8 ceramics, making lower operational power possible. However, the external $d c$ bias leads to decreased dielectric and piezoelectric properties and adds complexity and cost to the driving electronics. In addition, a rise in temperature, induced by the high mechanical loss of PMN-PT crystals, has been found to limit the pulse length and duty cycle. A

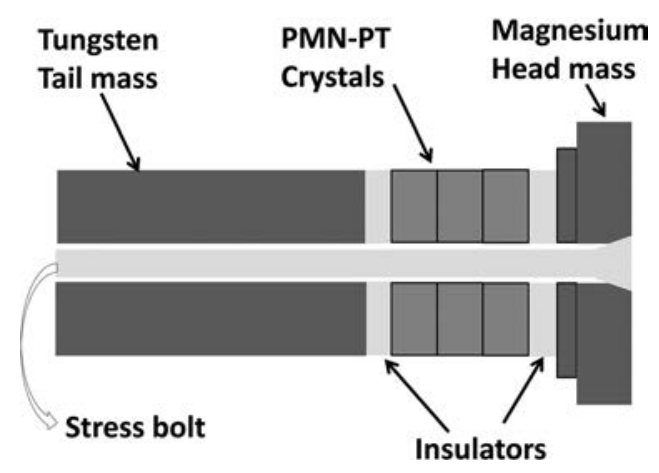

FIG. 48. Cross-section of typical tonpilz transducer. ${ }^{186}$

large mechanical preload via the bias bolt accelerates heating due to the strain and polarization nonlinearity. Furthermore, a significant change in dielectric permittivity as a function of temperature leads to significant strain on matching electronics and amplifiers, which will affect the power delivery. ${ }^{295}$

From a material viewpoint, the 2 nd generation relaxorPT single crystals offer a broader temperature usage range (less temperature dependent properties) and higher coercive fields (higher drive stability), while 3rd generation crystals with improved mechanical $Q_{m}$ (less heat generation and higher dynamic strain level) are important candidates for high power and broad bandwidth sonar transducer applications. It was reported that there was about five times reduction in the mechanical heat dissipation for the 3 rd generation crystals, when compared to PMN-PT counterpart. ${ }^{295}$

Transducers operating at low frequencies with high output power require large displacement of the radiating surfaces. The compact low frequency transducers typically employ novel mechanical system to generate additional displacement. In addition, some applications demand compromise between the compliance required to generate large displacements and the stiffness required to withstand hydrostatic pressure. Therefore, transducers exhibiting relatively high coupling and broadband response, which can withstand hydrostatic pressure in a compact form, would be desirable. Based on these requirements, crystals with different orientations and vibrations need to be considered, taking advantage of the strong anisotropic characteristics of single crystals. The use of [011] poled PMN-PT crystals in the "32" mode has been reported to allow lower drive and bias voltages, while simplifying construction with minimal impact on transducer effective coupling, size, and bandwidth. ${ }^{186,374,375}$ Compared to its " 33 " mode counterpart, the resonance frequency was decreased greatly, with an effective coupling of 0.83. ${ }^{377-379}$ Figure 49 shows a commercial MMT TP61 projector based on transverse extensional mode PZN-PT crystals, with an operating frequency range of $12-52 \mathrm{kHz}$, being very compact in size $(\sim 24 \mathrm{~mm}$ thick in the unpackaged form). ${ }^{159}$

Recently, the thickness shear vibration mode of relaxorPT crystals drew attentions for transducer applications, due to the high piezoelectric $d_{15}$, large electromechanical coupling $k_{15}$, and ultrahigh elastic compliance $s_{55}{ }^{E}$, allowing for ultralow frequency and broad bandwidth sonar transducers. $^{252}$ Strong cross talk between $d_{15}$ and $d_{16}$ exists in 


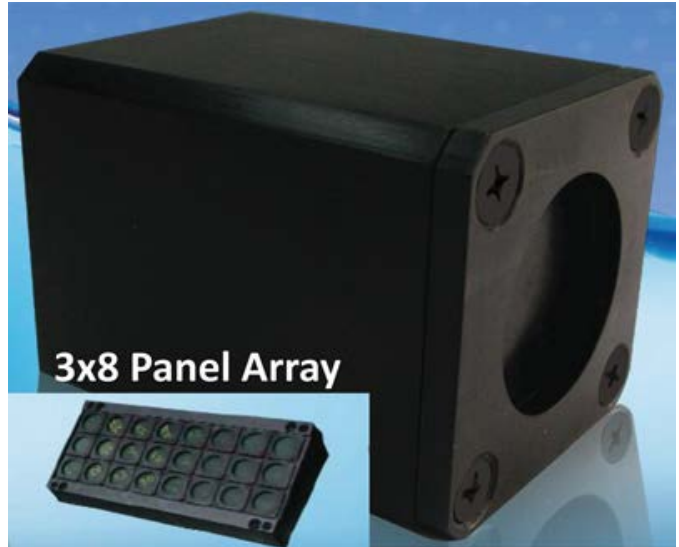

FIG. 49. (Color online) Projector TP16 operating in transverse extensional mode, fabricated by MMT (Courtesy of Dr. L. C. Lim from Microfine Materials Technologies). ${ }^{159}$

crystals with " $1 \mathrm{R}$ " single domain states, where $d_{16}$ can be eliminated by coordinate rotation, as discussed in Sec. III E 1. Of particular interest is that relaxor-PT crystals with " $2 \mathrm{R}$ " and "1O" engineered domain configurations were found to possess high shear piezoelectric $d_{15}$ and zero piezoelectric $d_{16}$, due to the macroscopic symmetry $\mathrm{mm} 2$, revealing these crystals are free of cross-talk, which will benefit the transducer design. In addition, the drive field stability $\left(<1 / 2 \mathrm{E}_{\mathrm{C}}\right.$ for 1 st and 2 nd generation crystals) can be improved greatly in the 3 rd generation crystals $\left(\sim 0.7 \mathrm{E}_{\mathrm{C}}\right)$, due to the existence of the internal bias field, which stabilizes the polarization and reduces the polarization rotation under external $a c$ drive field. ${ }^{326}$ The low mechanical $Q_{m}(\sim 30)$ of thickness shear components, however, limits their applications in projector type transducers. ${ }^{38}$ The face shear vibration mode of relaxorPT single crystals has been reported to be a potential candidate for low frequency and high power sonar transducer applications, due to the large piezoelectric $d_{36}$ and electromechanical coupling $k_{36} \cdot{ }^{252}$ Of particular importance is the large $a c$ drive field stability (being on the order of $\sim \mathrm{E}_{\mathrm{C}}$ ) and high mechanical $Q_{m}(>150)$, greatly increasing the allowable drive field and stress levels. ${ }^{253,254}$ In addition, the face shear mode offers the potential to minimize the transducer device size since the controlling dimension of the face shear is the edge length, which makes it possible to realize much lower operational frequencies when compared to the thickness shear mode devices. ${ }^{253}$

\section{B. Sensors}

Sensor is a device that responds to a physical stimulus and converts it into a signal which can be recorded. Basically, there are two types of sensors: active and passive sensors. Piezoelectric sensors are of the active type since no external source of power is required for measuring, while most other sensors are of the passive type, including capacitive, inductive, piezoresistive, and resistive sensors. ${ }^{393}$ Piezoelectric sensors are found to possess the advantages of high rigidity, high stability and reproducibility, fast response time, wide operating temperature range, and insensitivity to electric and magnetic fields, thus have been widely used for measuring force, torque, strain, pressure, acceleration, acoustic emission, etc. Materials used for piezoelectric sensors are expected to possess high piezoelectric sensitivity, high electrical resistivity, low temperature dependence of all properties within the operational temperature range, low loss (absence of hysteresis), high mechanical strength, etc. ${ }^{393}$ In the following, two typical piezoelectric sensors-hydrophone and accelerometer will be discussed, with emphasis on the recent developments using high performance relaxor-PT crystals.

\section{Hydrophones}

In most active sonar systems, the same transducer serves as both projector and hydrophone, but in some cases, such as towed line arrays for reception, separate hydrophones are used. $^{385}$

Hydrophones detect the pressure variation of acoustic signals and noise in water and produce an output voltage proportional to the pressure. Thus, the performance criteria for hydrophones are quite different from those for projectors, where projectors usually operate in the vicinity of resonance with power output the major concern, while hydrophones usually operate over a wide band below resonance, the output voltage, and signal-to-noise ratio are key. ${ }^{385}$

The response of pressure sensitive hydrophones is frequency independent and omnidirectional. In this way, the figure of merit of hydrophones can be defined as

$$
M^{2} C_{f}=g_{33} d_{33} V_{0},
$$

where $M$ is the voltage sensitivity and $C_{f}$ is the free capacitance and $V_{O}$ is the volume of the material, thus, the greater volume of piezoelectric material, the greater figure of merit. A basic limitation on hydrophone performance is the electrical noise generated internally by thermal agitation in its components and in water; this noise must not exceed the total sea noise for good performance. Electrical dissipation and mechanical resistance of the piezoelectric elements allow electrical and mechanical energy in the hydrophone to be lost as internal thermal energy, resulting in electrical noise. At far below the resonance frequency, the energy dissipation is mainly dominated by the dielectric loss of the piezoelectric material. An alternative figure of merit incorporating the noise generating loss factor, $\tan \delta$, will be considered and the above formula will be rewritten as

$$
M^{2} C_{f} / \tan \delta=g_{33} d_{33} V_{0} / \tan \delta,
$$

emphasizing the importance of a low dissipation factor. ${ }^{385}$ In this perspective, single crystal offers advantages over conventional soft PZTs, due to their much lower dielectric loss. In the case of hydrophone application, the state-of-art materials are lead metaniobate, which have high $g_{h}$ coefficients, due to the strong anisotropic piezoelectric characteristics. Although the resulting hydrostatic sensitivity is high for these materials, the dielectric permittivities are low, thus yielding a higher impedance and lower figure of merit. $^{385}$ 
TABLE X. Comparison of hydrostatic coefficients for various materials. ${ }^{186,398}$

\begin{tabular}{lrrr}
\hline \hline Material & \multicolumn{1}{c}{$\varepsilon_{\mathrm{r}}$} & $d_{h}(\mathrm{pm} / \mathrm{V})$ & $g_{h}(\mathrm{~V} \mathrm{~m} / \mathrm{N})$ \\
\hline PbNb $_{2} \mathrm{O}_{6}$ & 225 & 67 & 0.034 \\
PZT ceramics & 1800 & 40 & 0.0025 \\
PZT 1-3 composite & 54 & 27 & 0.056 \\
PMN-PT crystal & 4436 & 80 & 0.002 \\
[011] PIN-PMN-PT crystal “2 R" & 3000 & 87 & 0.003 \\
[011] PIN-PMN-PT crystal “1O” & 1500 & 110 & 0.008 \\
[001] PMN-PT 1-3 composite & 340 & 111 & 290 \\
[011] PMN-PT 2-2 composite & 800 & 337 & 910 \\
\hline \hline
\end{tabular}

It was reported that cymbal transducers, ${ }^{394}$ consisting of a piezoelectric disk sandwiched between two metal cymbalshaped endcaps, allow the radial motion of the piezoelectric material to be converted into a flextensional motion in the metal endcaps, leading to a large displacement. The single crystal cymbal hydrophone was reported to exhibit sensitivity $4 \mathrm{~dB}$ higher than that of PZT counterpart, taking advantage of the high $d_{31}$ and $d_{33}$ of relaxor-PT crystals. ${ }^{395}$ There has been a longtime interest in developing piezoelectric composites for underwater hydrophone applications because of their high hydrostatic sensitivity, good acoustic impedance matching to water, and high-pressure tolerance. ${ }^{385,396-399}$ Piezocomposite hydrophones are dominated by 1-3 type connectivity, in which, the arrangement of piezoelectric material and polymer will reduce the influence of the 31 and 32 modes and produces a significant improvement in hydrostatic voltage sensitivity, with a high figure of merit $d_{h} \cdot g_{h}{ }^{385,396}$ The composite design is intended to operate in thickness mode, with operational frequencies in the range of $\mathrm{Hz}$ to $\mathrm{MHz}$. Other engineered connectivities, such as parallel-connected 2-2 composites, have also been investigated, taking advantage of strong anisotropic behavior of [011] poled single crystals, with macroscopic mm2 symmetry. ${ }^{397-399}$ In this symmetry, the hydrostatic piezoelectric coefficient $d_{h}=d_{33}+d_{32}+d_{31}$, where $d_{33}$ and $d_{31}$ are both positive while $d_{32}$ is negative. As shown in Figure 44, when the polymer layers are perpendicular to the $\mathrm{Y}$ axis, the contribution of $d_{32}$ to the hydrostatic $d_{h}$ value will be greatly reduced, with minimal reduction of the $d_{33}$ and $d_{31}$ values, thus, the hydrostatic piezoelectric $d_{h}$ is significantly improved. It was reported that crystal/epoxy 2-2 composites with a crystal volume of $25 \%$, exhibited a large $d_{h}$ value, being on the order of $337 \mathrm{pC} / \mathrm{N} .{ }^{398}$ Table $\mathrm{X}$ lists the properties of various hydrostatic hydrophone materials, including polycrystalline ceramics, single crystals, 1-3 composites, and 2-2 crystal/epoxy composites, where the $2-2$ composites exhibit the most promising figure of merit $d_{h} \cdot g_{h}$, being on the order of $16000 \times 10^{-15} \mathrm{~m}^{2} / \mathrm{N} .^{186,385,398}$

\section{Accelerometers}

Piezoelectric accelerometers can be used to measure dynamic change in mechanical variables, such as acceleration, vibration, and mechanical shock. ${ }^{400-404}$ The state-of-art piezoelectrics for accelerometer applications are soft PZTs, depending on the operational temperature and frequency range. Considering the high piezoelectric coefficient and low dielectric loss of relaxor-PT single crystals, they are ideally suited for low noise and high sensitivity accelerometers. The accelerometer noise was reported to be inversely proportional to the charge output, especially at low frequencies. Platformed unimorph accelerometer using PMN-PT single crystals was developed by Wilcoxon Research Inc., exhibited a $15 \mathrm{~dB}$ reduction in its noise floor. ${ }^{402}$ Piezoelectric crystal accelerometers using PZN-PT have been developed at Microfine Material Technologies. ${ }^{159,404}$ Figure 50 shows the seismic/infrasonic accelerometers with $1 \mathrm{D}$ (right) and 3D (left) designs, which were reported to possess sensitivity being about $25000 \mathrm{pC} / \mathrm{g}$, or $2.5 \mathrm{~V} / \mathrm{g}$, and an extreme wide dynamic range from $<1 \times 10^{-5} \mathrm{~g}$ to $20 \mathrm{~g}$.

\section{Actuators}

Piezoelectric actuator is a device for positioning or controlling a system, operated by electric voltage or current, and converts it into motion. Due to the extreme accuracy of the controlling, piezoelectric actuators have been extensively used in a broad range of applications, including precise positioning, active damping, adaptive optics, adaptive structures for flap control, etc. ${ }^{186}$ Numerous types of piezoelectric actuators have been developed, including in-plane actuators, stack actuators, benders, flextensional actuators, ultrasonic motors (resonant actuators), etc. ${ }^{405-422}$ The state-of-art materials for off-resonance actuators are soft PZT ceramics, which possess piezoelectric strain coefficients up to

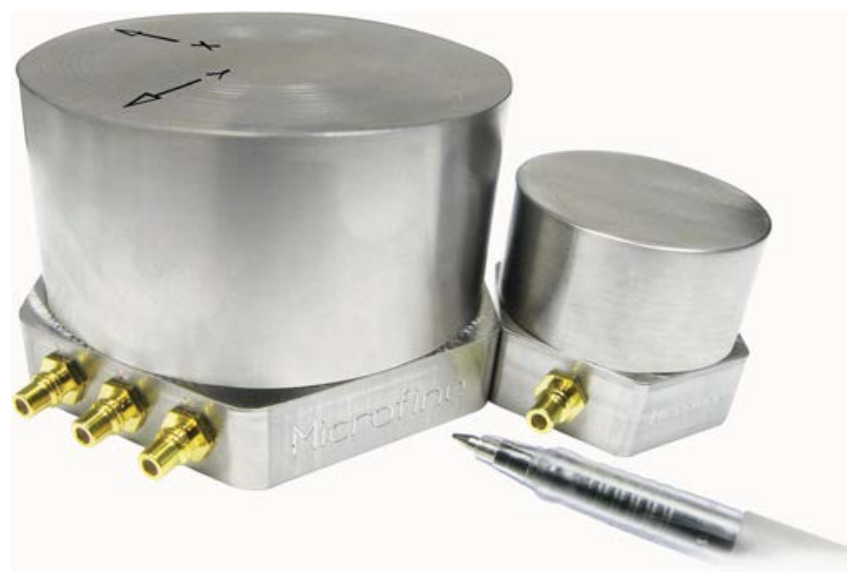

FIG. 50. (Color online) PZN-PT crystal based high sensitivity seismic/infrasonic accelerometer fabricated by MMT (Courtesy of Dr. L. C. Lim from Microfine Materials Technologies). ${ }^{159}$ 
TABLE XI. Properties comparison for various materials relate to actuator applications.

\begin{tabular}{|c|c|c|c|c|c|c|c|c|c|c|}
\hline Material & $\mathrm{T}_{\mathrm{C}}\left({ }^{\circ} \mathrm{C}\right)$ & $\mathrm{T}_{\mathrm{RT}}\left({ }^{\circ} \mathrm{C}\right)$ & $\mathrm{E}_{\mathrm{C}}(\mathrm{kV} / \mathrm{cm})$ & $\mathrm{E}_{\text {int }}(\mathrm{kV} / \mathrm{cm})$ & $\varepsilon_{\mathrm{r}}$ & $\tan \delta$ & $d_{33}(\mathrm{pm} / \mathrm{V})$ & $d_{33}^{*}(\mathrm{pm} / \mathrm{V})^{\mathrm{a}}$ & $\mathrm{Q}_{\mathrm{m}}$ & $d \cdot Q \times 10^{3}(\mathrm{pm} / \mathrm{V})$ \\
\hline \multicolumn{11}{|c|}{ Off_resonance actuator applications } \\
\hline PZT5A & 360 & - & 15 & - & 1700 & 0.02 & 380 & 650 & 75 & 30 \\
\hline PZT5H & 200 & - & 7.5 & - & 3400 & 0.02 & 600 & 750 & 75 & 45 \\
\hline PMN-PT29 & 135 & 96 & 2.3 & - & 5400 & 0.004 & 1540 & 1540 & 150 & 230 \\
\hline PMN-PT33 & 155 & 65 & 2.8 & - & 8200 & 0.004 & 2800 & 2800 & 100 & 280 \\
\hline PIN-PMN-PT & 197 & 96 & 5.5 & - & 7240 & 0.004 & 2740 & 2740 & 120 & 330 \\
\hline \multicolumn{11}{|c|}{ On-resonance actuator applications } \\
\hline PZT4 & 328 & - & 14.2 & 3.0 & 1300 & 0.004 & 300 & 380 & 500 & 150 \\
\hline PZT8 & 300 & - & 18.7 & 7.0 & 1000 & 0.004 & 230 & 230 & 1000 & 230 \\
\hline Mn:PIN-PMN-PT & 193 & 119 & 5.0 & 1.0 & 3700 & 0.002 & 1120 & 1120 & 810 & 910 \\
\hline Mn:PMN-PZT & 203 & 141 & 6.3 & 1.6 & 3410 & 0.002 & 1140 & 1140 & 1050 & 1200 \\
\hline
\end{tabular}

${ }^{a} d_{33}^{*}$ is calculated by the slope of $S-E$ loop, measured at $20 \mathrm{kV} / \mathrm{cm}$.

$\sim 600 \mathrm{pC} / \mathrm{N}$, while hard PZTs have been the mainstay for ultrasonic motors and/or resonance actuators, due to the large figure of merit $d \cdot Q$. However, many actuator applications, such as cryogenic temperature, low power consumption, large displacement, and high resolution, require advanced piezoelectric elements. Table XI list the principle properties of PZTs and PMN-PT single crystals, related to actuator applications. The piezoelectric coefficient of soft PZTs are in the range of $400-600 \mathrm{pC} / \mathrm{N}$, with large field piezoelectric coefficient $\mathrm{d}_{33}^{*}$ being on the order of $550-750 \mathrm{pC} / \mathrm{N}$. For comparison, the first generation PMN-PT single crystals offer $d_{33}$ being in the range of $\sim 1500 \mathrm{pC} / \mathrm{N}$ to $\sim 2800 \mathrm{pC} / \mathrm{N}$, depending on compositions. Of particular significance is that the high field $d_{33}{ }^{*}$ of PMN-PT crystals are found to be similar to the small signal values, with strain-field hysteresis less than $5 \%$, much smaller than that of soft PZTs, whose hysteresis values are about $>40 \%$. This is important for the high resolution actuation (precise positioning). In contrast, hard PZTs, such as PZT4 and PZT8, show high mechanical quality factor $Q_{m}$, with the $d \cdot Q$ values being on the order of $1-2 \times 10^{5} \mathrm{pm} / \mathrm{V}$, promising for resonant actuator applications. For comparison, $3 \mathrm{rd}$ generation single crystals offer high mechanical $Q_{m}$, being $>500$, with $d \cdot Q$ values being on the order of $10^{6} \mathrm{pm} / \mathrm{V}$, several times higher than their ceramic counterparts (Table XI). The Curie temperatures of PZTs are found to be $>200-360^{\circ} \mathrm{C}$, with suggested temperature usage ranges up to $100-250^{\circ} \mathrm{C}$. For comparison, the temperature usage range of single crystals is limited by the low rhombohedral (orthorhombic) to tetragonal ferroelectric phase transition temperatures, being on the order of $60-95^{\circ} \mathrm{C}$ for first generation crystals, improved to $>120^{\circ} \mathrm{C}$ for second generation crystals. It should be noted that relaxor-PT crystals exhibit high piezoelectric activity at cryogenic temperatures, with piezoelectric coefficient at $30 \mathrm{~K}$ comparable to the value of PZT ceramics at room temperature. $^{186}$

\section{Stack/in-plane actuators}

Piezoelectric actuators with low driving voltages $(<150 \mathrm{~V})$ are desired for some aerospace and industrial applications. Currently, widely used low voltage piezoelectric actuators are co-fired PZT actuators, with strain achieved less than $0.1 \%$, owing to the low piezoelectric coefficients. Single crystals exhibit much larger strain compared to conventional PZT ceramics, due to the ability to orientate the crystals along a preferred high strain crystallographic direction, thus, piezoelectric crystal stack actuators and in-plane actuators have been widely studied.

Stack actuators are featured with amplified stroke (each layer stroke times the number of layers) and high blocking force, while in-plane actuation mostly adopts the transverse mode to generate motion in plane. ${ }^{405-413}$

Stroke output of a stack actuator is given by the following formula:

$$
\Delta L=d_{33} \cdot \frac{V}{t} \cdot L
$$

where $\Delta L$ is the stroke, $d_{33}$ is the longitudinal piezoelectric coefficient, $V$ the driving voltage, $t$ the thickness of the crystal layer, and $L$ the active length of the stack (the sum of all active layer thickness). For stack actuators, [001] poled single crystals were used because of the ultrahigh piezoelectric coefficient $>1500 \mathrm{pC} / \mathrm{N}$. The complete stack is composed of crystal plates, metal shim, endcaps, and epoxy bonding layers, which are assembled as schematically shown in Figure $51 .{ }^{407,409,410}$ It was reported that the stacked actuators provide a factor of two higher strain energy density, a factor of ten higher strain level $(>1 \%)$, and a lower loss in heat dissipation due to improved hysteretic behavior, when compared to PZT multilayer actuators. ${ }^{406-410}$

For in-plane actuators, [011] poled single crystals have been used, taking advantage of large transverse piezoelectric coefficients $-d_{32}$, being $>1200 \mathrm{pC} / \mathrm{N}$, with large in-plane displacement along the [100] direction. In addition, performance enhancement has been achieved for in-plane actuators by utilizing inter-digital electrodes (IDEs). Maximum microstrains of 886 (@15kV/cm) have been reported with the PZN-PT single crystal IDE devices, compared with 250 microstrain (@8kV/cm) from the IDE devices using PZT5A. ${ }^{413}$

Displacement $>50 \mu \mathrm{m}$ was reported in $20 \mathrm{~mm}$ long single crystal stack actuators with low dielectric loss $(<0.1 \%)$ and low hysteresis under the driving field of $14 \mathrm{kV} / \mathrm{cm}$, while in-plane stroke of $24 \mu \mathrm{m}$ has been achieved 


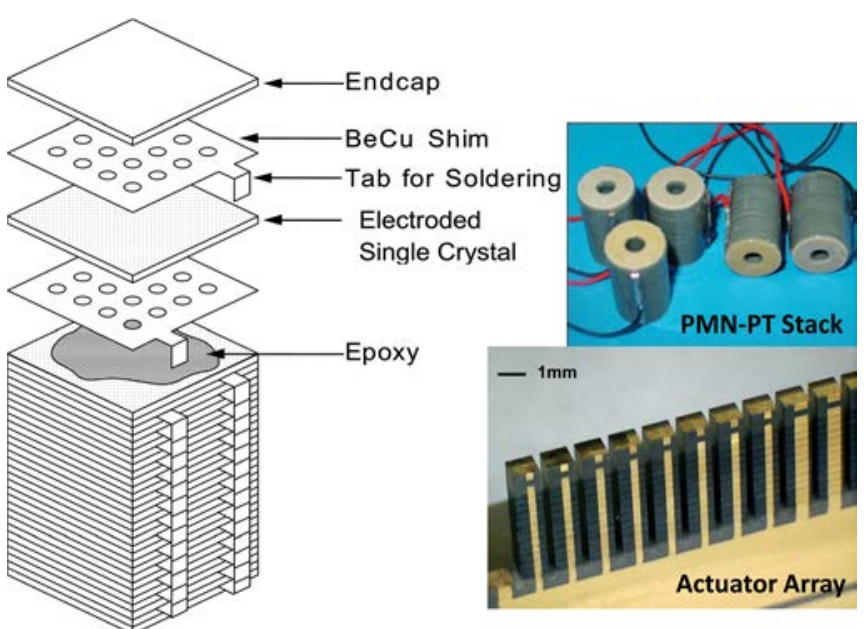

FIG. 51. (Color online) Schematic view of single crystal stack actuator assembly ${ }^{407}$ and crystal stack/actuator array fabricated by TRS Technologies ${ }^{199}$ (Courtesy of R. Sahul from TRS Technologies).

with $15 \times 6 \times 0.5 \mathrm{~mm}$ single crystal plates at driving field of $8 \mathrm{kV} / \mathrm{cm}$. The results show that the single crystal piezoelectric actuators hold enhanced stroke under lower driving field when compared to PZT counterparts, while keeping the nature of piezoelectric actuations with high precision, high resolution $(<3 \mathrm{~nm})$, and fast response under high preload stress. The potential benefits of these crystal actuators in deformable mirrors have been demonstrated at cryogenic temperatures by TRS Technologies, for large throw deformable mirror in space telescopes and airborne wavefront control systems. ${ }^{199,406-410}$

\section{Flextensional actuators}

Flextensional actuators such as "moonie," "cymbal," "thunder," etc. are well known with low profile and large stroke due to the compliance amplification mechanism, but their force output is low compared to stack actuators. ${ }^{186,199,406-409,412,413}$ Two different piezoelectric drivers can be used for flextensional actuators (cymbal type). Figure 52 (left) shows a typical "31" mode flextensional actuators, where the driving element is a piezoelectric plate and its horizontal " 31 " strain will be amplified into vertical actuation, while Figure 52 (right) gives the "33" mode flextensional actuators, where the stack actuator is inserted into a thin metal frame (amplification mechanism).
Taking advantage of the strong anisotropic characteristics of single crystals, the piezoelectric element used in " 32 " mode flextensional actuators are poled along [011] direction, with the length direction along [100], while [001] poled crystal plates are used in the "33" mode flextensional actuators. It was reported that a large stroke over $66 \mu \mathrm{m}$ has been achieved under $400 \mathrm{~V}$ in "32" mode flextensional actuator, using PMN-PT crystal plate with dimension of $15 \times 6 \times 0.5 \mathrm{~mm}$, while over $100 \mu \mathrm{m}$ stroke was obtained under $500 \mathrm{~V}$ for " 33 " mode flextensional actuator. Both actuators showed fast response time, being $0.1-0.15 \mathrm{msec}$. Furthermore, the stroke of the flextensional actuators was found to remain $>60 \%$ of the room temperature values at a cryogenic temperature of $\sim 70 \mathrm{~K} .^{406,413}$

\section{Ultrasonic motors (Resonant actuators)}

At resonance frequency, the strain $(x)$ of piezoelectric materials is given by

$$
x=\frac{8}{\pi^{2}} Q_{m} d \cdot E,
$$

where it can be seen that the strain is proportional to the figure of merit $d \cdot Q$. For resonance based actuators, in addition to a high piezoelectric strain, attention must be paid to the mechanical loss (inverse of mechanical quality factor $Q_{m}$ ). The needs are essentially focused on high $d \cdot Q$ figure of merit in order to obtain high strain level near the resonance frequency, the medium depoling stress, and the coefficients' linearity. ${ }^{414-422}$

Piezoelectric motor is a type of the resonant actuator, which operates on ultrasonic wave/friction drive mechanism. It has high displacement resolution and frictional locking in the power-off state, while possessing the advantage of being driven by an electric field, without parasitic magnetic fields as for conventional electromagnetic motors. Thus, piezoelectric motor is a good candidate for high precision micromechanical systems, such as camera phones, micro robots, and medical endoscopes. ${ }^{414-417}$ The piezoelectric materials used for commercial ultrasonic motors are "hard" PZTs, with high figure of merit $d \cdot Q$, exhibiting high power and high driving force, however, PZT based piezoelectric motors are limited to the temperature range of $-40{ }^{\circ} \mathrm{C}$ (performance reduction and increased hysteretic losses) to $100^{\circ} \mathrm{C}$ (thermal instability and depoling), which is not practical for low temperature
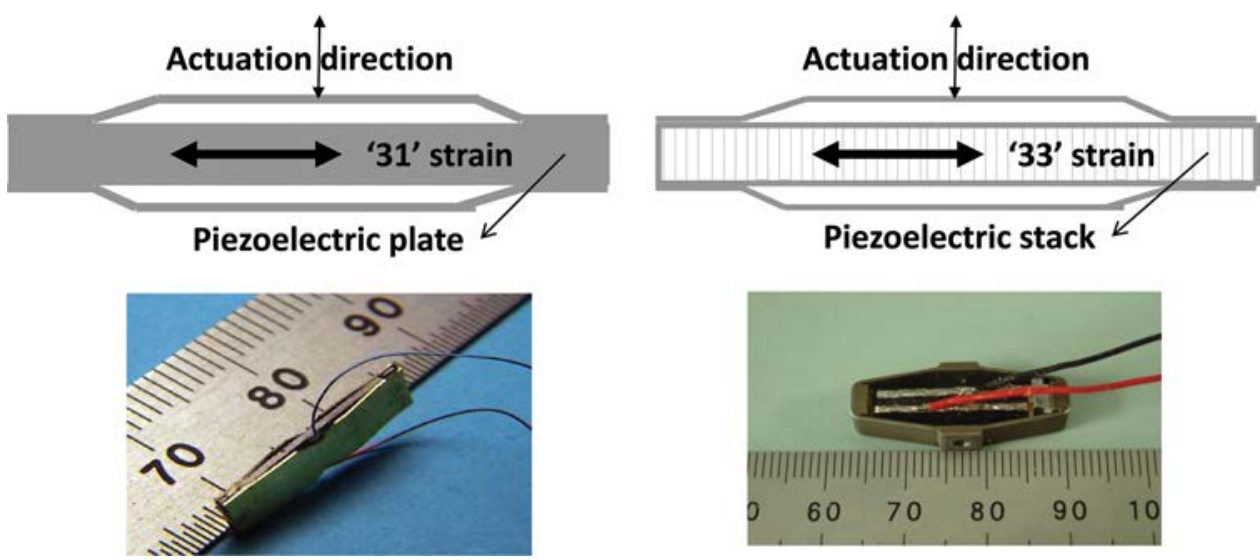

FIG. 52. (Color online) Flextensional actuators in "31" and "33" modes, fabricated by TRS Technologies. ${ }^{199,406,409}$ 
applications, such as adaptive structure drives for shape, position, and force controls in spaceborne and airborne platforms. ${ }^{417}$ PMN-PT single crystal based linear/rotatory ultrasonic motors were reported, with a large torque, high resolution, and low power consumption. A single crystal ultrasonic motor based on wobbling mode with center coupling is shown in Figure 53..$^{414,417}$ Two single crystal ring stacks were fabricated with segmented electrodes to apply one pair of voltage signal with a $\pi / 2$ phase difference, and a "wobbling motion" at the center part of the stator was excited with high efficiency. This linear motor shown the features of low driving voltage $\sim 60-70 \mathrm{~V}_{\mathrm{pp}}$, high traveling speed $50-100 \mathrm{~mm} / \mathrm{sec}$, fast direction reverse, with maximum driving force on the order of $200 \mathrm{~g}$, which was also demonstrated being operational at low temperature of $\sim 77 \mathrm{~K} .{ }^{417}$

Compared to conventional PZT4 based ultrasonic motor, PMN-PT crystal based motor show larger amplitude of the stator, due to higher $d \cdot Q$ for PMN-PT crystals. Meanwhile, a large $d$ coefficient gives rise to lower exciting voltage and larger output torque, while the low $Q_{m}$ leads to wider exciting frequency range. However, the low $Q_{m}$ of PMN-PT crystals results in significant heat generation in the ultrasonic motor, thus, both high $d$ and high $Q_{m}$ are desirable for the motor applications, where the 3 rd generation single crystals should be considered as the piezoelectric element in motor design.

It should be noted that the mechanical $Q_{m}$ at antiresonance frequency was reported to be higher than the value at resonance frequency. ${ }^{420}$ Furthermore, the impedance reaches maximum value at antiresonance frequency, requires low current and high voltage driving, in contrast to the high cur-

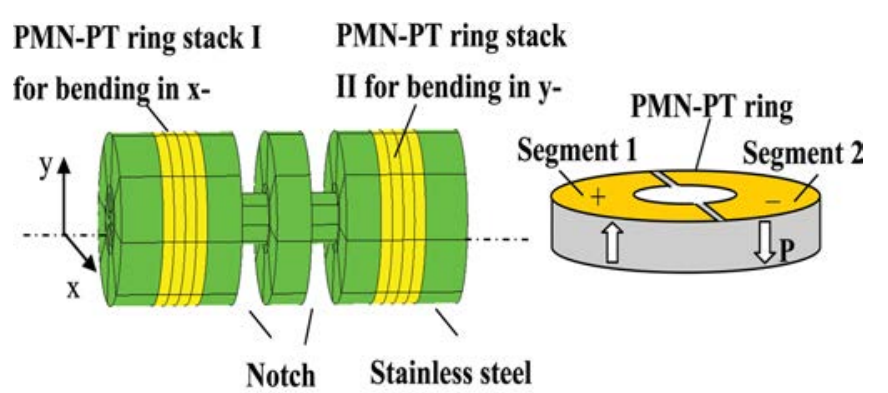

(a)

(b)

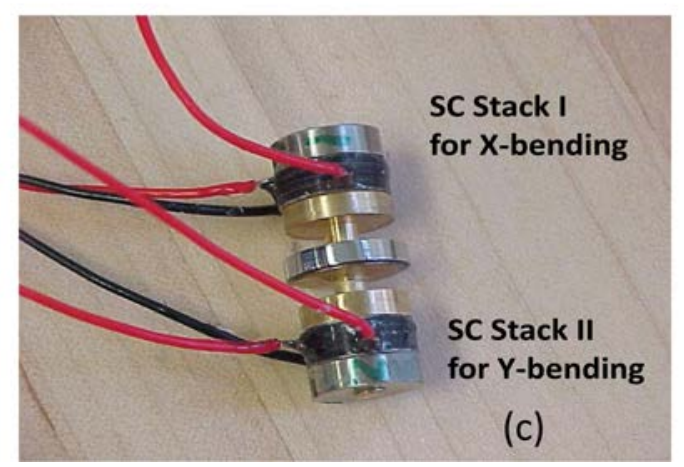

FIG. 53. (Color online) (a) Piezoelectric composite stator with two notches, (b) PMN-PT crystal ring, and (c) the assembled piezoelectric stator. Reprinted with permission from X. N. Jiang, in IEEE Ultrasonic Symposium (2004), pp. 1314-1317; S. X. Dong et al., Applied Physics Letters 86, 053501 (2005). Copyright (C 2005, the American Institute of Physics. rent and low voltage for the "resonance" drive, which means that a conventional inexpensive power supply may be utilized as driving source for the antiresonance based devices. ${ }^{420}$ Thus, ultrasonic motors working at antiresonance frequency and using 3rd generation crystals are expected to exhibit improved performance.

In summary, single crystals show great potential for high performance ultrasonic transducers, sensors and actuators, far out-perform the state-of-art polycrystalline ceramics. Besides the above electromechanical devices, relaxor-PT single crystals have also been found for other applications, such as piezoelectric transformers, ${ }^{296,423}$ piezoelectric energy harvesting, ${ }^{42-427}$ pyroelectric energy harvesting, ${ }^{428,429}$ and thermal imaging, ${ }^{268,269,430,431}$ which make relaxor-PT crystals "materials of choice" for next generation devices.

\section{SUMMARY AND FUTURE PERSPECTIVE}

\section{A. Summary}

In summary, three generations of relaxor-PT single crystals have been surveyed in this article. The high temperature solution, Bridgman, and solid state crystal growth techniques were reviewed in relation to the growth of large size and commercially viable crystals. Currently, PMN-PT and PINPMN-PT single crystals up to $100 \mathrm{~mm}$ in diameter and $200 \mathrm{~mm}$ in length along [001] growth direction have been successfully commercialized using modified multi-crucible Bridgman method.

In contrast to polycrystalline ceramics, relaxor-PT crystals possess not only ultrahigh piezoelectric/electromechanical properties but also high tunability of related properties. Different piezoelectric/electromechanical properties can be achieved in relaxor-PT crystals, taking advantage of engineered domain configurations, crystal phase, orientation, and anisotropic characteristics, which are not achievable in polycrystalline ceramics.

A facilitated polarization rotation, being related to the high shear deformation of the lattice, is deemed responsible for the high longitudinal piezoelectric coefficients of relaxorPT crystals. The MPBs and relaxor end member are thought to be critical factors for structural instability, leading to the facilitated polarization rotation (i.e., high shear piezoelectric response) of relaxor-PT crystals. However, the ease in polarization rotation also induces high mechanical loss observed in relaxor-PT crystals.

The Curie temperature $T_{C}$ and ferroelectric phase transition temperatures $T_{R T}$ are key parameters that affect the properties and usage range of relaxor-PT crystals. ${ }^{432}$ Figure 54 shows the room temperature piezoelectric coefficient, electromechanical coupling factor, and coercive field for [001] poled relaxor-PT based ferroelectric single crystals as a function of $T_{R T}$ and/or $T_{C}$. The piezoelectric coefficients are found to decrease with increasing $T_{R T}$, but not $T_{C}$ as reported for polycrystalline PZT ceramics (as evident in Figure 2(a)). In contrast to the piezoelectric coefficients, the electromechanical couplings are found to be independent of either $T_{C}$ or $T_{R T}$, where $k_{33}$ values for various relaxor-PT crystal systems with MPB compositions are on the order of $\sim 0.9$, regardless of their transition temperatures. In addition, the coercive fields 

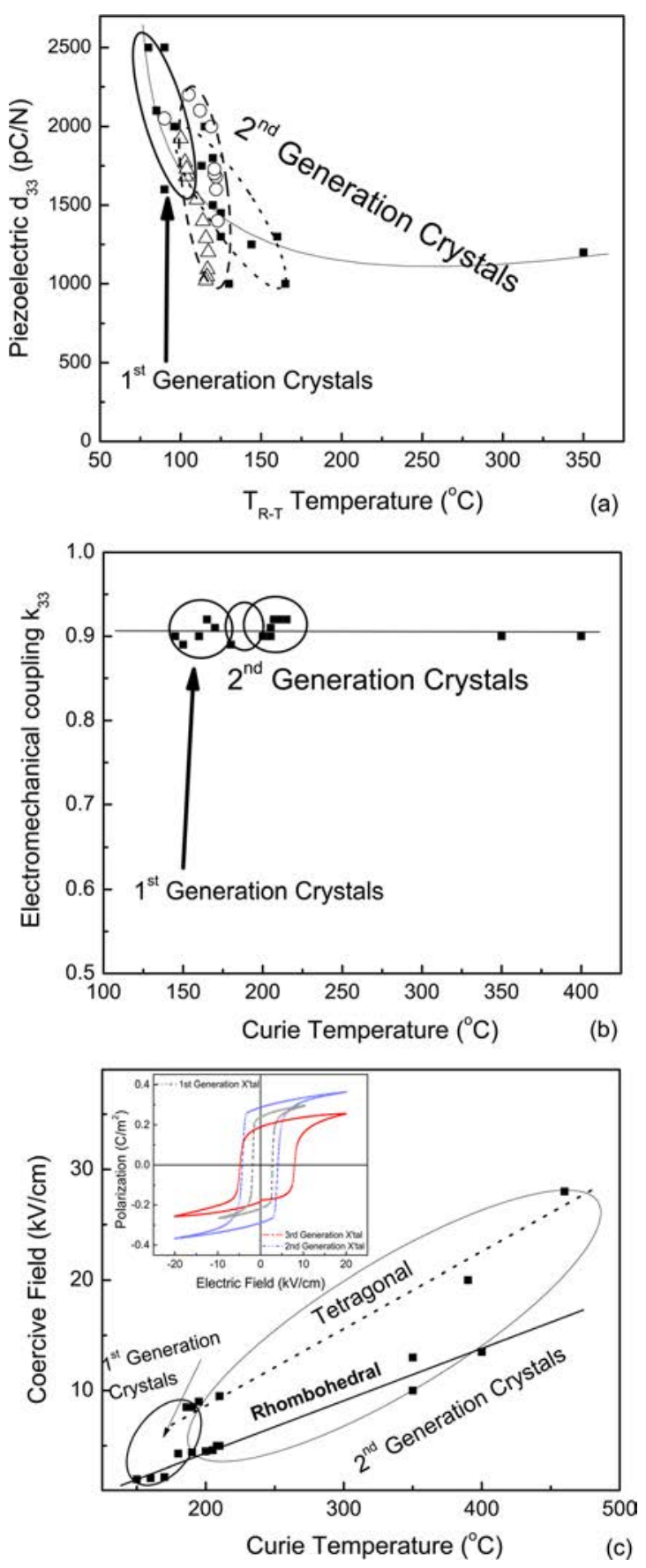

FIG. 54. (Color online) (a) Piezoelectric coefficient as a function of $\mathrm{T}_{\mathrm{RT}}$; (b) Electromechanical coupling as function of $\mathrm{T}_{\mathrm{C}}$; and (c) Coercive field as function of $\mathrm{T}_{\mathrm{C}}$; for various relaxor-PT crystal systems. The small inset shows polarization hysteresis of $1 \mathrm{st}$, 2nd, and $3 \mathrm{rd}$ generations crystals. Reprinted with permission from S. J. Zhang and T. R. Shrout, IEEE Transactions on Ultrasonics Ferroelectrectrics Frequency Control 57, 2138 (2010). Copyright $@ 2010$, IEEE.

of ferroelectric crystals are found to increase with increasing $T_{C}$, as shown in Figure 54(c). It is of interest to note that for the same crystal system, tetragonal compositions are found to possess significantly higher coercive fields when compared to their rhombohedral counterparts, being associated with their respective domain structures. The second generation crystals, including ternary PIN-PMN-PT and PMN-PZT are found to possess enhanced $T_{C}$ and $T_{R T}$ when compared to first generation binary crystals, offering improved thermal stability and an overall broadened temperature usage range. The polarization-electric field behavior for various crystal systems are given in the small inset of Figure 54(c), where the PMNPZT (second generation crystal) is found to offer nearly twice of $E_{C}$ of binary PMN-PT (first generation), being on the order of $\sim 5 \mathrm{kV} / \mathrm{cm}$ versus $2.3 \mathrm{kV} / \mathrm{cm}$. The Mn modified PMN-PZT (third generation) shows increased $E_{C}(\sim 6.3 \mathrm{kV} / \mathrm{cm})$ when compared to its pure counterpart, with an internal bias field being on the order of $1.6 \mathrm{kV} / \mathrm{cm}$, due to the fact that the acceptor-oxygen vacancy defect dipoles clamp domain wall motion and inhibit polarization rotation.

For polycrystalline ceramics, a consequence of high $Q_{m}$ is the sacrifice of electromechanical couplings, As shown in Figure 55, the coupling $k_{33} s$ are found to decrease with increasing mechanical $Q_{m}$ values. Thus, both high $Q_{m}$ and high coupling cannot be simultaneously achieved in polycrystalline ceramics. Of particular significance is that for domain engineered relaxor-PT crystal systems, in contrast to ceramics, the mechanical $Q_{m}$ values can be improved without sacrificing the electromechanical couplings, as shown in Figure 55, the modified crystal systems exhibit different levels of $Q_{m}$, in the range of 70-2000, while maintaining ultrahigh electromechanical couplings, being on the order of $>0.85$, demonstrating relaxor-PT crystals are unique piezoelectrics and the "materials of choice" for next generation electromechanical applications. ${ }^{432}$

Innovations in electromechanical device design continue to be the motivation for the development and exploration of new piezoelectric materials. Three main application areas of the relaxor-PT single crystals have been reviewed in this article, including ultrasound transducers, sensors, and actuators, with comparison to state-of-art polycrystalline ceramics. PMN-PT single crystals have been commercialized for the medical ultrasonic transducers and cryogenic actuators, owing to their ultrahigh electromechanical couplings and piezoelectric properties, while second and third generation

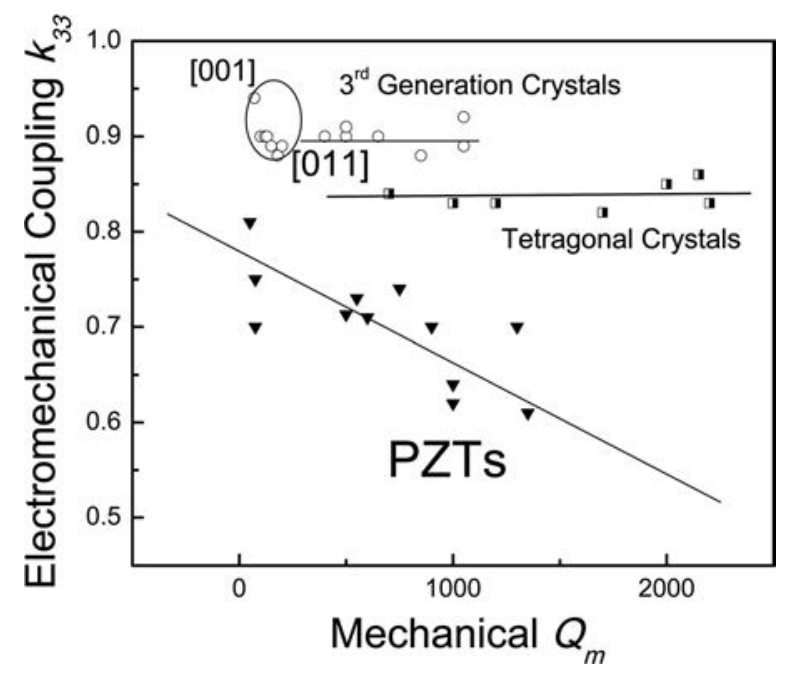

FIG. 55. The relationship between mechanical quality factor $Q_{m}$ and electromechanical coupling factor for different polycrystalline and single crystal systems. Reprinted with permission from S. J. Zhang and T. R. Shrout, IEEE Transactions on Ultrasonics Ferroelectrectrics Frequency Control 57, 2138 (2010). Copyright $\odot 2010$, IEEE. 
crystals offer the possibilities in the applications where high thermal and field stabilities are desired.

\section{B. Future perspectives}

In this section, we leave the readers with a few queries and perspectives

(a) How to decrease the cost of relaxor-PT single crystals and the property variations? The continuous feeding Bridgman method is expected to offer improved compositional uniformity and increased yield of the as-grown crystal boules.

(b) How to increase the temperature usage range by modifying the MPB? Can the curvature of MPB be understood?

(c) For the third generation crystals, what is the role of the donor and/or acceptor modifications? What is the defect dipole direction in crystals before and after the poling process? Will the poling condition affects the defect dipole? What is the relationship between the defect dipoles and domain wall motion/polarization rotation?

(d) How to control/utilize the nonlinear behavior of the crystals under external stress, electric field, and thermal field? How they affect the device performance? What is the drive limitation for high power applications?

(e) How to take advantage of the anisotropic characteristics of single crystals? For example, new crystal cuts, including thickness shear $d_{24}$ in "1O" single domain state and longitudinal $d_{33}$ in " 30 " engineered domain state showing high thermal stability, face shear $d_{36}$ exhibiting high mechanical $Q_{m}$, and high field stability.

\section{ACKNOWLEDGMENTS}

The authors want to thank Professor Thomas R. Shrout for his valuable comments and suggestions on this review paper and strong support for the authors' research. Also thanks to Dr. Jun Luo and Dr. Wesley S. Hackenberger from TRS Technologies for offering single crystals and the helpful discussions, Professor Xiaoning Jiang from North Carolina State University for the helpful discussion. The transducer array in the cover image is the courtesy of Dr. Xuecang Geng from Blatek Inc. Dr. Wally A. Smith, Dr. Carl C. M. Wu, and Dr. Ming-Jen Pan are acknowledged for their supports. One of the authors, F. Li also would like to thank National Natural Science Foundation of China under Grant Nos. 51102193 and 10976022. This work was supported by ONR under Grant Nos. N00014-07-C-0858 and N00014-09-01-0456.

${ }^{1}$ B. Jaffe, W. R. Cook, and H. Jaffe, Piezoelectric Ceramics (Academic, New York, 1971).

${ }^{2}$ A. J. Moulson and J. M. Herbert, Electroceramics (Wiley, London, 2003).

${ }^{3}$ S. E. Park and T. R. Shrout, J. Appl. Phys. 82, 1804 (1997).

${ }^{4}$ H. Fu and R. E. Cohen, Nature 403, 281 (2000).

${ }^{5}$ M. Davis, J. Electroceram. 19, 23 (2007).

${ }^{6}$ N. Setter, D. Damjanovic, L. Eng, G. Fox, S. Gevorgian, S. Hong, A. Kingon, H. Kohlstedt, N. Y. Park, G. B. Stephenson, I. Stolitchnov, A. K. Taganstev, D. V. Taylor, T. Yamada, and S. Streiffer, J. Appl. Phys. 100, 051606 (2006)

${ }^{7}$ D. Damjanovic, Rep. Prog. Phys. 61, 1267 (1998).

${ }^{8}$ S. Trolier-Mckinstry and P. Muralt, J. Electroceram. 12, 7 (2004).

${ }^{9}$ G. L. Messing, S. Trolier-McKinstry, E. M. Sabolsky, C. Duran, S. Kwon, B. Brahmaroutu, P. Park, H. Yilmaz, P. W. Rehrig, K. B. Eitel, E.
Suvaci, M. Seabaugh, and K. S. Oh, Crit. Rev. Solid State Mater. Sci. 29, 45 (2004).

${ }^{10}$ Q. M. Zhang, V. Bharti, and X. Zhao, Science 280, 2101 (1998).

${ }^{11}$ R. E. Newnham, D. P. Skinner, and L. E. Cross, Mater. Res. Bull. 13, 525 (1978).

${ }^{12}$ W. A. Smith and B. A. Auld, IEEE. Trans. Ultrason. Ferroelectr. Freq. Control 38, 40 (1991).

${ }^{13}$ W. A. Smith and A. A. Shaulov, Ferroelectrics 87, 309 (1988).

${ }^{14}$ R. E. Newnham, Ann. Rev. Mater. Sci. 16, 47 (1986).

${ }^{15}$ J. Curie and P. Curie, Bull. Soc. Fr. Mineral. 3, 90 (1880).

${ }^{16}$ G. Lippman, Ann. Chim. Phys. 24, 145 (1881).

${ }^{17} \mathrm{~N}$. Setter, "ABC of piezoelectricity and piezoelectric materials," in Piezoelectric Materials in Devices, edited by N. Setter (Lausanne, Switzerland, 2002), pp. 1-27.

${ }^{18}$ R. E. Newnham, Properties of Materials-Anisotropy, Symmetry, Structure (Oxford University Press, NY, 2005).

${ }^{19}$ D. Berlincourt, "Piezoelectric crystals and ceramics," in Ultrasonic Transducer Materials: Piezoelectric Crystals and Ceramics, edited by O. E. Mattiat (Plenum, London, 1971), pp. 63-124.

${ }^{20} \mathrm{Y} . \mathrm{H} . \mathrm{Xu}$, Ferroelectric Materials and their Applications (Elsevier, NY, 1991).

${ }^{21}$ W. G. Cady, Piezoelectricity (Dover, NY, 1964).

${ }^{22}$ T. Ikeda, Fundamentals of Piezoelectricity (Oxford University Press, NY, 1990).

${ }^{23}$ W. P. Mason, Piezoelectric Crystals and their Application to Ultrasonics (Van Nostrand Co., New York, 1950).

${ }^{24}$ D. Berlincourt, D. R. Curran, and H. Jaffe, "Piezoelectric and piezomagnetic materials and their function in transducers," in Physical Acoustics, edited by W. P. Mason (Academic, New York, 1964), Vol. IA, pp. $169-270$.

${ }^{25}$ A. Safari and E. K. Akdogan, Piezoelectric and Acoustic Materials for Transducer Applications (Springer, NY, 2008).

${ }^{26}$ K. Uchino, Ferroelectric Devices (CRC, NY, 2009).

${ }^{27}$ M. E. Lines and A. M. Glass, Principles and Applications of Ferroelectrics and Related Materials (Clarendon, Oxford, 1977).

${ }^{28}$ L. E. Cross, "Ferroelectric ceramics: Tailoring properties for specific applications," in Ferroelectric Ceramics: Tutorial Reviews, Theory, Processing and Applications, edited by N. Setter and E. L. Colla (Birkhauser, Basel, 1993), pp. 1-87.

${ }^{29}$ J. Valasek, Phys. Rev. 17, 475 (1921).

${ }^{30} \mathrm{~S}$. Wada, "Domain wall engineering in piezoelectric crystals with engineered domain configuration," in Handbook of Advanced Dielectric, Piezoelectric and Ferroelectric Materials - Synthesis, Characterization and Applications, edited by Z. G. Ye (Woodhead, Cambridge, England, 2008), pp. 266-303.

${ }^{31} \mathrm{~W}$. W. Cao, "Full-set material properties and domain engineering principles of ferroelectric single crystals," in Handbook of Advanced Dielectric, Piezoelectric and Ferroelectric Materials - Synthesis, Characterization and Applications, edited by Z. G. Ye (Woodhead, Cambridge, England, 2008), pp. 235-265.

${ }^{32}$ J. Erhart, Phase Transitions 77, 989 (2004).

${ }^{33} \mathrm{M}$. Davis, "Phase transitions, anisotropy and domain engineering: The piezoelectric properties of realxor-ferroelectric single crystals," $\mathrm{Ph} . \mathrm{D}$. dissertation (Swiss Federal Institute of Technology-EPFL, Lausanne, Switzerland, 2006).

${ }^{34} \mathrm{~K}$. G. Webber, "Effect of domain wall motion and phase transformations on nonlinear hysteretic constitutive behavior in ferroelectric materials," $\mathrm{Ph}$. D. dissertation (Georgia Institute of Technology, 2008).

${ }^{35}$ T. Q. Liu, "Electromechanical behavior of relaxor ferroelectric crystals," Ph.D. dissertation (Georgia Institute of Technology, 2004).

${ }^{36}$ D. Damjanovic, "Hysteresis in piezoelectric and ferroelectric materials," in The Science of Hysteresis, edited by I. Mayergoyz and G. Bertotti (Elsevier, NY, 2005), Vol. 3, pp. 337-465.

${ }^{37}$ F. Li, S. Zhang, Z. Xu, X. Wei, J. Luo, and T. Shrout, Appl. Phys. Lett. 97, 252903 (2010).

${ }^{38}$ F. Li, S. J. Zhang, Z. Xu, X. Y. Wei, and T. R. Shrout, Adv. Funct. Mater. 21, 2118 (2011).

${ }^{39}$ A. J. Bell, J. Appl. Phys. 89, 3907 (2001).

${ }^{40}$ M. Davis, D. Damjanovic, D. Hayem, and N. Setter, J. Appl. Phys. 98, 014102 (2005).

${ }^{41}$ S. Wada, K. Yato, H. Kakemoto, T. Tsurumi, and T. Kiguchi, J. Appl. Phys. 98, 014109 (2005).

${ }^{42}$ Y. Yamashita, N. Yamamoto, K. Itsumi, and Y. Hosono, Jpn. J. Appl. Phys. 50, 09NC05 (2011). 
${ }^{43}$ D. Lin, S. J. Zhang, Z. Li, F. Li, Z. Xu, S. Wada, J. Luo, and T. R. Shrout, J. Appl. Phys. 100, 084110 (2011).

${ }^{44}$ E. Wainer and A. N. Salomon, Titanium Alloy. Mfg. Co. Elect. Report No. 8, 1942.

${ }^{45}$ A. V. Hippel, R. Breckenridge, F. Chesley, and L. Tisza, Ind. Eng. Chem. 38, 1097 (1946).

${ }^{46}$ R. E. Newnham and L. E. Cross, Mater. Res. Bull. 30, 845 (2005).

${ }^{47}$ B. Jaffe, R. Roth, and S. Marzullo, J. Appl. Phys. 25, 809 (1954).

${ }^{48}$ G. H. Haetling, J. Am. Ceram. Soc. 82, 797 (1999).

${ }^{49}$ D. Berlincourt, C. Cmolik, and H. Jaffe, Proc. IRE. 48, 220 (1960).

${ }^{50}$ G. A. Smolenskii and A. I. Agranovskii, Sov. Phys. Solid State 1, 1429 (1960).

${ }^{51}$ A. A. Bokov and Z. G. Ye, J. Mater. Sci. 41, 31 (2006).

${ }^{52}$ Z. G. Ye, Key Eng. Mater. 155, 81 (1998).

${ }^{53}$ S. J. Jang, K. Uchino, S. Nomura, and L. E. Cross, Ferroelectrics 27, 31 (1979).

${ }^{54}$ L. E. Cross, Ferroelectrics 76, 241 (1987).

${ }^{55}$ S. W. Choi, T. R. Shrout, S. J. Jang, and A. S. Bhalla, Mater. Lett. 8, 253 (1989).

${ }^{56}$ S. L. Swartz and T. R. Shrout, Mater. Res. Bull. 17, 1245 (1982).

${ }^{57}$ J. Kuwata, K. Uchino, and S. Nomura, Ferroelectrics 37, 579 (1981).

${ }^{58}$ J. Kuwata, K. Uchino, and S. Nomura, Jpn. J. Appl. Phys. 21, 1298 (1982).

${ }^{59}$ T. R. Shrout, Z. P. Chang, N. Kim, and S. Markgraf, Ferroelectr., Lett. Sect. 12, 63 (1990)

${ }^{60}$ Z. G. Ye, P. Tissot, and H. Schmid, Mater. Res. Bull. 25, 739 (1990).

${ }^{61}$ S. E. Park and T. R. Shrout, IEEE Trans. Ultrason. Ferroelectr. Freq. Control. 44, 1140 (1997).

${ }^{62}$ S. E. Park and T. R. Shrout, Mater. Res. Innovations 1, 20 (1997).

${ }^{63}$ Z. G. Ye, Curr. Opin. Solid State Mater. Sci. 6, 35 (2002).

${ }^{64}$ S. E. Park and W. Hackenberger, Curr. Opin. Solid State Mater. Sci. 6, 11 (2002).

${ }^{65}$ S. J. Zhang, L. Lebrun, L, S. F. Liu, S. Rhee, C. A. Randall, and T. R. Shrout, Jpn. J. Appl. Phys. 41, L1099 (2002).

${ }^{66}$ R. Zhang. B. Jiang, W. H. Jiang, and W. W. Cao, IEEE Trans. Ultrason. Ferroelectr. Freq. Control 49, 1622 (2002).

${ }^{67}$ S. J. Zhang, L. Lebrun, D. Y. Jeong, C. A. Randall, Q. M. Zhang, and T. R. Shrout, J. Appl. Phys. 93, 9257 (2003).

${ }^{68}$ S. J. Zhang, L. Lebrun, C. A. Randall, and T. R. Shrout, Phys. Status Solidi A 202, 151 (2005).

${ }^{69}$ M. Shanthi, K. H. Hoe, C. Y. H. Lim, and L. C. Lim, Appl. Phys. Lett. 86, 262908 (2005)

${ }^{70}$ S. J. Zhang, J. Luo, R. Xia, P. W. Rehrig, C. A. Randall, and T. R. Shrout, Solid State Commun. 137, 16 (2006).

${ }^{71}$ R. Zhang, B. Jiang, and W. W. Cao, J. Appl. Phys. 90, 3471 (2001).

${ }^{72}$ R. Zhang, B. Jiang, and W. W. Cao, Appl. Phys. Lett. 82, 787 (2003).

${ }^{73}$ M. Davis, D. Damjanovic, and N. Setter, Phys. Rev. B 73, 014115 (2006).

${ }^{74}$ H. Dammak, A. Renault, P. Gaucher, M. Thi, and G. Calvarin, Jpn. J. Appl. Phys. 42, 6477 (2003).

${ }^{75}$ D. Damjanovic, M. Davis, and M. Budimir, "Enhancement of piezoelectric properties in perovskite crystals by thermally, compositionally, electric field and stress-induced instabilities," in Handbook of Advanced Dielectric, Piezoelectric and Ferroelectric Materials - Synthesis, Characterization and Applications, edited by Z. G. Ye (Woodhead, Cambridge, England, 2008), pp. 304-332.

${ }^{76}$ S. J. Zhang, J. Luo, D. W. Snyder, and T. R. Shrout, "High performance, high temperature piezoelectric crystals," in Handbook of Advanced Dielectric, Piezoelectric and Ferroelectric Materials - Synthesis, Characterization and Applications, edited by Z. G. Ye (Woodhead, Cambridge, England, 2008), pp. 130-157.

${ }^{77}$ Y. Yamashita and K. Harada, Jpn. J. Appl. Phys. 36, 6039 (1997).

${ }^{78}$ Y. H. Bing and Z. G. Ye, J. Cryst. Growth 250, 118 (2003).

${ }^{79}$ Y. H. Bing and Z. G. Ye, J. Cryst. Growth 287, 326 (2006).

${ }^{80}$ Y. H. Bing and Z. G. Ye, Mater. Sci. Eng. B 120, 72 (2005).

${ }^{81}$ Y. Yamashita and S. Shimanuki, Mater. Res. Bull. 31, 887 (1996).

${ }^{82}$ Y. Yamashita, Y. Hosono, K. Harada, and Z. G. Ye, "Relaxor Ferroelectric crystals-recent development and application," in Piezoelectric Materials in Devices, edited by N. Setter (Lausanne, Switzerland, 2002), pp. 455-466.

${ }^{83}$ Y. H. Bing and Z. G. Ye, "Piezo-and ferroelectric (1-x)PSN-xPT solid solution system," in Handbook of Advanced Dielectric, Piezoelectric and Ferroelectric Materials - Synthesis, Characterization and Applications, edited by Z. G. Ye (Woodhead, Cambridge, England, 2008), pp. 173-204.

${ }^{84}$ Y. Guo, H. Luo, T. He, and Z. Yin, Solid State Commun. 123, 417 (2002).

${ }^{85}$ N. Yasuda, H. Ohwa, M. Kume, K. Hayashi, Y. Hosono, and Y. Yamashita, J. Cryst. Growth 229, 299 (2001)

${ }^{86} \mathrm{~N}$. Yasuda, H. Ohwa, M. Kume, and Y. Yamashita, Jpn. J. Appl. Phys. 39, L66 (2000).

${ }^{87}$ N. Yasuda, N. Mori, H. Ohwa, Y. Hosono, Y. Yamashita, M. Iwata, M. Maeda, I. Suzuki, and Y. Ishibashi, Jpn. J. Appl. Phys. 41, 7007 (2002).

${ }^{88}$ Y. Guo, H. Luo, T. He, X. Pan, and Z. Yin, Mater. Res. Bull. 38, 857 (2003).

${ }^{89}$ Z. Q. Duan, G. S. Xu, X. F. Wang, D. F. Yang, X. M. Pan, and P. C. Wang, Solid State Commun. 134, 559 (2005).

${ }^{90}$ N. Yasuda, H. Ohwa, M. Kume, Y. Hosono, Y. Yamashita, S. Ishino, H. Terauchi, M. Iwata, and Y. Ishibashi, Jpn. J. Appl. Phys. 40, 5664 (2001).

${ }^{91}$ S. J. Zhang, P. W. Rehrig, C. A. Randall, and T. R. Shrout, J. Cryst. Growth 234, 415 (2002).

${ }^{92}$ S. J. Zhang, S. Priya, E. Furman, T. Shrout and C. Randall, J. Appl. Phys., 91, 6002 (2002).

${ }^{93}$ S. J. Zhang, S. Rhee, C. A. Randall, and T. R. Shrout, Jpn. J. Appl. Phys. 41, 722 (2002).

${ }^{94}$ S. Zhang, L. Lebrun, S. Rhee, C. Randall, and T. R. Shrout, Appl. Phys. Lett. 81, 892 (2002).

${ }^{95}$ S. J. Zhang, C. A. Randall, and T. R. Shrout, Jpn. J. Appl. Phys. 43, 6199 (2004).

${ }^{96}$ S. J. Zhang, C. A. Randall, and T. R. Shrout, Jpn. J. Appl. Phys. 42, L1152 (2003).

${ }^{97}$ S. J. Zhang, C. A. Randall, and T. R. Shrout, J. Appl. Phys. 95, 4291 (2004).

${ }^{98}$ S. J. Zhang, L. Lebrun, S. Rhee, R. E. Eitel, C. A. Randall, and T. R. Shrout, J. Cryst. Growth 236, 210 (2002).

${ }^{99}$ S. J. Zhang, C. A. Randall, and T. R. Shrout, Solid State Commun. 131, 41 (2004).

${ }^{100}$ S. J. Zhang, C. A. Randall, and T. R. Shrout, IEEE Trans. Ultrason. Ferroelectr. Freq. Control. 52, 564 (2005).

${ }^{101}$ S. J. Zhang, C. A. Randall, and T. R. Shrout, Appl. Phys. Lett. 83, 3150 (2003).

${ }^{102}$ Y. Hosono, K. Harada, Y. Yamashita, M. Dong, and Z. G. Ye, Jpn. J. Appl. Phys. 39, 5589 (2000).

${ }^{103}$ Y. J. Yamashita and Y. Hosono, "High Curie temperature piezoelectric single crystals of the PIN-PMN-PT ternary materials system," in Handbook of Advanced Dielectric, Piezoelectric and Ferroelectric Materials Synthesis, Characterization and Applications, edited by Z. G. Ye (Woodhead, Cambridge, England, 2008), pp. 205-234.

${ }^{104}$ Y. Hosono, Y. Yamashita, H. Sakamoto, and N. Ichinose, Jpn. J. Appl. Phys. 42, 5681 (2003).

${ }^{105}$ Y. Hosono, Y. Yamashita, H. Sakamoto, and N. Ichinose, Jpn. J. Appl. Phys. 41, L1240 (2002).

${ }^{106} \mathrm{~J}$. Luo, W. Hackenberger, S. Zhang, and T. Shrout, in IEEE Ultrasonic Symposium (IEEE, Piscataway, NJ, 2008), pp. 261-264.

${ }^{107}$ G. S. Xu, K. Chen, D. F. Yang, and J. B. Li, Appl. Phys. Lett. 90, 032901 (2007).

${ }^{108}$ J. Tian, P. D. Han, X. L. Huang, and H. X. Pan, Appl. Phys. Lett. 91, 222903 (2007).

${ }^{109}$ S. J. Zhang, J. Luo, W. Hackenberger, and T. R. Shrout, J. Appl. Phys. 104, 064106 (2008).

${ }^{110}$ X. Z. Liu, S. J. Zhang, J. Luo, T. R. Shrout, and W. W. Cao, J. Appl. Phys. 106, 074112 (2009).

${ }^{111}$ S. J. Zhang, J. Luo, W. Hackenberger, N. P. Sherlock, R. J. Meyer, Jr., and T. R. Shrout, J. Appl. Phys. 105, 104506 (2009).

${ }^{112}$ F. Li, S. J. Zhang, Z. Xu, X. Y. Wei, J. Luo, and T. Shrout, J. Appl. Phys. 107, 054107 (2010).

${ }^{113}$ F. Li, S. Zhang, Z. Xu, X. Y. Wei, J. Luo, and T. Shrout, J. Am. Ceram. Soc. 93, 2731 (2010).

${ }^{114}$ E. W. Sun, S. J. Zhang, J. Luo, T. R. Shrout, and W. W. Cao, Appl. Phys. Lett. 97, 032902 (2010).

${ }^{115}$ X. Z. Liu, S. J. Zhang, J. Luo, T. R. Shrout, and W. W. Cao, Appl. Phys. Lett. 96, 012907 (2010).

${ }^{116}$ F. Li, S. J. Zhang, D. B. Lin, J. Luo, Z. Xu, X. Y. Wei, and T. R. Shrout, J. Appl. Phys. 109, 014108 (2011).

${ }^{117}$ S. J. Zhang, F. Li, N. P. Sherlock, J. Luo, H. J. Lee, R. Xia, R. J. Meyer, Jr., W. Hackenberger, and T. R. Shrout, J. Cryst. Growth 318, 846 (2011). 
${ }^{118}$ P. Finkel, H. Robinson, J. Stace, and A. Amin, Appl. Phys. Lett. 97, 122903 (2010).

${ }^{119}$ P. Zhao, S. Goljahi, W. Dong, T. Wu, P. Finkel, R. Sahul, K. Snook, J. Luo, W. Hackenberger, and C. S. Lynch, Smart Mater. Struct. 20, 055006 (2011).

${ }^{120}$ P. Finkel, K. Benjamin, and A. Amin, Appl. Phys. Lett. 98, 192902 (2011)

${ }^{121}$ W. Wang, D. Liu, Q. H. Zhang, B. Ren, Y. Y. Zhang, J. Jiao, D. Lin, and H. S. Luo, J. Appl. Phys. 107, 084101 (2010).

${ }^{122}$ Y. Y. Zhang, D. A. Liu, Q. H. Zhang, W. Wang, B. Ren, X. Y. Zhao, and H. S. Luo, J. Electr. Mater. 40, 92 (2011).

${ }^{123}$ Y. Y. Zhang, X. B. Li, D. A. Liu, Q. H. Zhang, W. Wang, B. Ren, D. Lin, X. Y. Zhao, and H. S. Luo, J. Cryst. Growth 318, 890 (2011).

${ }^{124}$ E. Sun, W. Cao, W. Jiang, and P. Han, Appl. Phys. Lett. 99, 032901 (2011)

${ }^{125}$ D. Zhou, K. Cheung, K. Lam, Y. Chen, Y. Chiu, J. Dai, H. Chan, and H. Luo, Rev. Sci. Instrum. 82, 055110 (2011).

${ }^{126}$ J. Gao, Z. Xu, F. Li, C. Zhang, Y. Liu, G. Liu, and H. He, Appl. Phys. Lett. 99, 062903 (2011).

${ }^{127}$ S. Zhang, S. Lee, D. Kim, H. Lee, and T. Shrout, J. Appl. Phys. 102, 114103 (2007)

${ }^{128}$ S. Zhang, S. Lee, D. Kim, H. Lee, and T. Shrout, Appl. Phys. Lett. 90, 232911 (2007)

${ }^{129}$ A. Amin, H. Y. Lee, and B. Kelly, Appl. Phys. Lett. 90, 242912 (2007).

${ }^{130}$ S. Zhang, S. Lee, D. Kim, H. Lee, and T. Shrout, J. Am. Ceram. Soc. 90, 3859 (2007).

${ }^{131}$ Z. G. Xia, Q. Li, and S. F. Zhang, Solid State Commun. 145, 38 (2008).

${ }^{132}$ Q. Li, Y. L. Zhang, Z. G. Xia, and X. C. Chu, J. Cryst. Growth 318, 851 (2011).

${ }^{133}$ W. A. Smith, continuing advocacy of the fundamental studies on ferroelectric materials for more than 20 years, first proposed the nomenclature of the varieties of piezocrystals at Navy Workshop on Acoustic Transduction Materials and Devices, State College, PA, 11-13 May 2010.

${ }^{134}$ S. J. Zhang, L. Lebrun, C. A. Randall, and T. R. Shrout, J. Cryst. Growth 267, 204 (2004).

${ }^{135}$ S. Priya, H. W. Kim, J. H. Ryu, S. J. Zhang, T. R. Shrout, and K. Uchino, J. Appl. Phys. 92, 3923 (2002).

${ }^{136}$ S. J. Zhang, S. M. Lee, D. H. Kim, H. Y. Lee, and T. R. Shrout, Appl. Phys. Lett. 93, 122908 (2008).

${ }^{137}$ N. P. Sherlock, S. J. Zhang, J. Luo, H. Y. Lee, T. R. Shrout, and R. J. Meyer, Jr., J. Appl. Phys. 107, 074108 (2010).

${ }^{138}$ D. T. J. Huerle, Handbook of Crystal Growth, Bulk Crystal Growth Vol. 2 (Elsevier, NY, 1994).

${ }^{139}$ J. Y. Xu and M. Jin, New Type Relaxor Ferroelectric Crystals (Chemical Industry Press, Beijing, 2007) [in Chinese].

${ }^{140}$ I. E. Myl'nikova and V. A. Bokov, Kristallografiya 4, 443 (1959) [in Russian]

${ }^{141}$ V. A. Bokov and I. E. Myl'nikova, Sov. Phys. Solid State 2, 2428 (1961).

${ }^{142}$ S. Nomura, T. Takahashi, and Y. Yokomizo, Jpn. J. Appl. Phys. 27, 262 (1969).

${ }^{143}$ Y. Yokomizo, T. Takahashi, and S. Nomura, J. Phys. Soc. Jpn. 28, 1278 (1970)

${ }^{144}$ N. Setter and L. E. Cross, J. Cryst. Growth 50, 555 (1980).

${ }^{145}$ L. C. Lim, M. Shanthi, K. K. Rajan, and C. Y. H. Lim, J. Cryst. Growth 282, 330 (2005).

${ }^{146}$ L. C. Lim, "Flux growth of PZN-PT and PMN-PT single crystals," in Piezoelectric Single Crystals and Their Applications, edited by S. TrolierMcKinstry, L. E. Cross, and Y. Yamashita (Pennsylvania State University, State College, 2004), pp. 117-137.

${ }^{147}$ L. C. Lim, "Flux growth and characterization of PZN-PT and PMN-PT single crystals," in Handbook of Advanced Dielectric, Piezoelectric and Ferroelectric Materials - Synthesis, Characterization and Applications, edited by Z. G. Ye (Woodhead, Cambridge, England, 2008), pp. 38-72.

${ }^{148}$ M. L. Mulvihill, S. E. Park, G. Risch, Z. Li, K. Uchino, and T. R. Shrout, Jpn. J. Appl. Phys. 35, 3984 (1996).

${ }^{149}$ S. Saitoh, T. Kobayashi, K. Harada, S. Shimanuki, and Y. Yamashita, IEEE Trans. Ultrason. Ferroelectr. Freq. Control 45, 1071 (1998).

${ }^{150}$ S. Saitoh, T. Takeuchi, T. Kobayashi, K. Harada, S. Shimanuki, and Y. Yamashita, IEEE Trans. Ultrason. Ferroelectr. Freq. Control 46, 414 (1999).

${ }^{151}$ D. Elwell and H. J. Scheel, Crystal Growth from High-Temperature Solutions (Academic, NY, 1975).
${ }^{152}$ F. J. Kumar, L. C. Lim, C. Chilong, and M. J. Tan, J. Cryst. Growth 216 , 311 (2000).

${ }^{153}$ G. Sandrine, R. Gilles, S. Nava, and R. Jean-Pierre, Ann. Chim. Sci. Mater. 26, 107 (2001).

${ }^{154}$ L. C. Lim, R. Liu, and F. J. Kumar, J. Am. Ceram. Soc. 85, 2817 (2002).

${ }^{155}$ L. C. Lim, F. J. Kumar, and A. Amin, J. Appl. Phys. 93, 3671 (2003).

${ }^{156}$ X. J. Wu, J. Y. Xu, X. H. Li, M. Jin, A. H. Wu, M. L. Shi, and S. J. Fan, Mater. Chem. Phys. 99, 220 (2006)

${ }^{157}$ M. Dong and Z. G. Ye, Jpn. J. Appl. Phys. 40, 4604 (2001).

${ }^{158}$ S. J. Zhang, D. Y. Jeong, Q. M. Zhang, and T. R. Shrout, J. Cryst. Growth 247, 131 (2003).

${ }^{159}$ See http://www.microfine-piezo.com for "World leader in PZN-PT single crystals and devices."

${ }^{160}$ K. Harada, S. Shimanuki, T. Kobayashi, S. Saitoh, and Y. Yamashita, J. Am. Ceram. Soc. 81, 2785 (1998).

${ }^{161}$ S. Shimanuki, S. Saitoh, and Y. Yamashita, Jpn. J. Appl. Phys. 37, 3382 (1998).

${ }^{162}$ K. Harada, Y. Hosono, S. Saitoh, and Y. Yamashita, Jpn. J. Appl. Phys. 39, 3117 (2000).

${ }^{163}$ K. Harada, Y. Hosono, Y. Yamashita, and K. Miwa, J. Cryst. Growth 229, 294 (2001).

${ }^{164}$ S. Shimanuki, S. Saito, and Y. Yamashita, Jpn. J. Appl. Phys. 37, 3382 (1998).

${ }^{165}$ M. Matsushita, T. Tachi, and K. Echizenya, J. Cryst. Growth 237-239, 853 (2002).

${ }^{166}$ B. J. Fang, H. Q. Xu, T. H. He, H. S. Luo, and Z. W. Yin, J. Cryst. Growth 244, 318 (2002).

${ }^{167}$ J. Y. Xu, S. J. Fan, B. Lu, J. Tong, and A. Zhang, Jpn. J. Appl. Phys. 41, 7000 (2002).

${ }^{168}$ J. Y. Xu, J. Tong, M. Shi, A. Wu, and S. J. Fan, J. Cryst. Growth 253, 274 (2003).

${ }^{169}$ J. Y. Xu, X. Wu, J. Tong, M. Shi, and G. Qian, J. Cryst. Growth 280, 107 (2005).

${ }^{170}$ X. J. Wu, J. Y. Xu, M. L. Shi, J. Tong, S. J. Fan, A. H. Wu, X. H. Li, X. H. Zeng, X. Yao, and X. H. Zeng, Mater. Sci. Eng. B 117, 129 (2005).

${ }^{171}$ A. Benayad, D. Kobor, L. Lebrun, B. Guiffard, and D. Guyomar, J. Cryst. Growth 270, 137 (2004).

${ }^{172}$ W. Chen and Z. G. Ye, J. Cryst. Growth 233, 503 (2001).

${ }^{173}$ W. Chen and Z. G. Ye, J. Mater. Sci. 36, 4393 (2001).

${ }^{174}$ R. Bertram, G. Reck, and R. Uecker, J. Cryst. Growth 253, 212 (2003).

${ }^{175}$ T. Karaki, M. Nakamoto, and M. Adachi, Jpn. J. Appl. Phys. 41, 6997 (2002).

${ }^{176}$ W. A. Bonner and L. G. V. Uitert, Mater. Res. Bull. 2, 131 (1967).

${ }^{177}$ P. W. Bridgman, Proc. Am. Acad. Arts Sci. 60, 305 (1925).

${ }^{178}$ D. C. Stockbarger, Rev. Sci. Instrum. 7, 133 (1936).

${ }^{179}$ D. T. J. Hurle, Prog. Mater. Sci. 10, 79 (1962).

${ }^{180}$ W. G. Pfann, Trans. AIME 194, 747 (1952).

${ }^{181}$ K. T. Zawilski, R. C. DeMattei, and R. S. Feigelson, J. Cryst. Growth 277, 393 (2005).

${ }^{182}$ W. G. Pfann, Zone Melting (Wiley, NY, 1966), pp. 8-27.

${ }^{183}$ H. Luo, G. Shen, P. Wang, X. Le, and Z. Yin, J. Inorg. Mater. 12, 768 (1997).

${ }^{184}$ R. S. Feigelson, "Growth of large single crystals of relaxor ferroelectrics under controlled conditions," in Proceedings of the Piezoelectric Crystal Planning Workshop, Washington, DC, 14-16 May 1997.

${ }^{185}$ P. Han, J. Tian, and W. Yan, "Bridgman growth and properties of PMNPT based single crystals," in Handbook of Advanced Dielectric, Piezoelectric and Ferroelectric Materials - Synthesis, Characterization and Applications, edited by Z. G. Ye (Woodhead, Cambridge, England, 2008), pp. 3-37.

${ }^{186}$ W. Hackenberger, J. Luo, X. N. Jiang, K. A. Snook, P. W. Rehrig, S. J. Zhang, and T. R. Shrout, "Recent developments and applications of piezoelectric crystals," in Handbook of Advanced Dielectric, Piezoelectric and Ferroelectric Materials - Synthesis, Characterization and Applications, edited by Z. G. Ye (Woodhead, Cambridge, England, 2008), pp. 73-100.

${ }^{187}$ P. Han, "Growth and characterization of large PMN-PT crystals," in Proccedings of the US Navy Workshop on Acoustic Transduction Materials and Devices, State College, PA, 13-15 April 1999.

${ }^{188}$ P.M. Bridenbaugh, J. Rottenberg, and G. Ruland, "Single crystal growth of PMN-PT," in Proccedings of the US Navy Workshop on Acoustic Transduction Materials and Devices, State College, PA, 13-15 April 1999. 
${ }^{189}$ S.G. Lee, R. Monteiro, M.C. Custodio, and R.S. Feigelson, "Growth of PMN and PMN-PT by the vertical Bridgman method," in Proccedings of the US Navy Workshop on Acoustic Transduction Materials and Devices, State College, PA, 13-15 April 1999.

${ }^{190}$ A. Benayad, G. Sebald, L. Lebrun, B. Guiffard, S. Pruvost, D. Guyomar, and L. Beylat, Mater. Res. Bull. 41, 1069 (2006).

${ }^{191}$ Z. W. Yin, H. S. Luo, P. C. Wang, and G. S. Xu, Ferroelectrics 229, 207 (1999).

${ }^{192}$ H. S. Luo, G. S. Xu, P. C. Wang, and Z. W. Yin, Ferroelectrics 231, 97 (1999).

${ }^{193}$ K. T. Zawilski, M. C. C. Custodio, R. C. DeMattei, S. G. Lee, R. G. Monteiro, H. Odagawa, and R. F. Feigelson, J. Cryst. Growth 258, 353 (2003).

${ }^{194}$ G. Xu, H. Luo, H. Xu, Z. Qi, P. Wang, W. Zhong, and Z. Yin, J. Cryst. Growth 222, 202 (2001).

${ }^{195} \mathrm{P}$. Han and J. Tian, "Progress in growth of 3-inch diameter PMN-PT crystals," in Proceedings of the US Navy Workshop on Acoustic Transduction Materials and Devices, State College, PA, 9-11 May 2006.

${ }^{196} \mathrm{P}$. Han, "Progress in commercialization of large sized (up to 4 inch diameter) PMN-PT based piezoelectric crystals," in Proccedings of the US Navy Workshop on Acoustic Transduction Materials and Devices, State College, PA, 13-15 May 2008.

${ }^{197}$ J. Luo, W. Hackenberger, S. J. Zhang and T. R. Shrout, "Progresses in manufacturing large size binary and ternary relaxor piezoelectric single crystals," in Proceedings of the US Navy Workshop on Acoustic Transduction Materials and Devices, State College, PA, 13-15 May 2008.

${ }^{198}$ J. Luo, S. J. Zhang, W. Hackenberger and T. R. Shrout, "Bridgman growth and characterization of relaxor-PT piezoelectric crystals," in Proceedings of the US Navy Workshop on Acoustic Transduction Materials and Devices, State College, PA, 11-13 May 2010.

${ }^{199}$ See http://www.trstechnologies.com for "A leader in the development of high performance materials."

${ }^{200}$ See http://www.hcmat.com for "A leader in the development and manufacture of high-performance single crystals."

${ }^{201}$ K. Echizenya, M. Matsushita, Y. Tachi, and T. Addona, "Characterization of PMN-PT single crystals grown by continuous feed technique," in proceedings of the US Navy Workshop on Acoustic Transduction Materials and Devices, State College, PA, 11-13 May 2010.

${ }^{202}$ J. Luo, W. Hackenberger, S. J. Zhang, and T. R. Shrout, "An update on development and manufacture of relaxor-based piezoelectric crystals," in proceedings of the International Workshop on Acoustic Transduction Materials and Devices, State College, PA, 10-12 May 2011.

${ }^{203}$ P. D. Han, J. Tian, and H. Pan, "Auto-feeding Bridgman method for growth of large single crystals of PMN-PT and PIN-PMN-PT solid solutions," in Proceedings of the International Workshop on Acoustic Transduction Materials and Devices, State College, PA, 10-12 May 2011.

${ }^{204}$ T. Yamamoto and T. Sakuma, J. Am. Ceram. Soc. 77, 1107 (1994).

${ }^{205}$ Y. S. Yoo, M. K. Kang, J. H. Han, H. Kim, and D. Y. Kim, J. Eur. Ceram. Soc. 17, 1725 (1997)

${ }^{206}$ P. W. Rehrig, G. L. Messing, and S. Trolier-McKinstry, J. Am. Ceram. Soc. 83, 2654 (2000).

${ }^{207}$ H. Y. Lee, J. S. Kim, and D. Y. Kim, J. Eur. Ceram. Soc. 20, 1595 (2000)

${ }^{208}$ T. Li, A. M. Scotch, H. M. Chan, and M. P. Harmer, J. Am. Ceram. Soc. 81, 244 (1998)

${ }^{209}$ A. Khan, F. A. Meschke, T. Li, A. M. Scotch, H. M. Chan, and M. P. Harmer, J. Am. Ceram. Soc. 82, 2958 (1999).

${ }^{210}$ E. M. Sabolsky, A. R. James, S. Kwon, S. Trolier-McKinstry, and G. L. Messing, Appl. Phys. Lett. 78, 2551 (2001).

${ }^{211}$ E. M. Sabolsky, S. Trolier-McKinstry, and G. L. Messing, J. Appl. Phys. 93, 4072 (2003).

${ }^{212}$ K. H. Brosnan, S. F. Poterala, R. J. Meyer, S. Misture, and G. L. Messing, J. Am. Ceram. Soc. 92, S133 (2009).

${ }^{213}$ K. H. Brosnan, G. L. Messing, R. J. Meyer, and M. D. Vaudin, J. Am. Ceram. Soc. 89, 1965 (2006)

${ }^{214}$ H. Y. Lee, H. M. Chan, and M. P. Harmer, J. Korean Ceram. Soc. 35, 905 (1998)

${ }^{215}$ H. Y. Lee, "Solid-state single crystal growth method: A cost-effective way of growing piezoelectric single crystals," in Piezoelectric Single Crystals and Their Applications, edited by S. Trolier-McKinstry, L. E. Cross, and Y. Yamashita (Pennsylvania State University, State College, 2004), pp. 160-177.

${ }^{216}$ H. Y. Lee, "Development of high performance piezoelectric single crystals by using solid-state single crystal growth (SSCG) method," in Hand- book of Advanced Dielectric, Piezoelectric and Ferroelectric Materials Synthesis, Characterization and Applications, edited by Z. G. Ye (Woodhead, Cambridge, England, 2008), pp. 158-172.

${ }^{217}$ See http://Ceracomp.com for "Korea supplier of piezoelectric single crystals."

${ }^{218}$ B. Noheda, Curr. Opin. Solid State Mater. Sci. 6, 27 (2002).

${ }^{219}$ J. F. Nye, Physical Properties of Crystals (Clarendon, Oxford, 1976).

${ }^{220}$ G. S. Xu, H. S. Luo, H. Q. Xu, and Z. W. Yin, Phys. Rev. B 64, 020102 (2001).

${ }^{221}$ R. R. Chien, V. H. Schmidt, L.-W. Hung, and C. Tu, J. Appl. Phys. 97, $114112(2005)$.

${ }^{222}$ J. J. Yao, Y. D. Yang, W. W. Ge, J. F. Li, and D. Viehland, J. Am. Ceram. Soc. 94, 2497 (2011).

${ }^{223}$ C.-S. Tu, R. Chien, F.-T. Wang, V. H. Schmidt, and P. Han, Phys. Rev. B 70, 220103 (2004).

${ }^{224}$ D. B. Lin, Z. R. Li, S. J. Zhang, Z. Xu, and X. Yao, J. Appl. Phys. 108, 034112 (2010).

${ }^{225}$ F. Li, S. J. Zhang, Z. Xu, X. Wei, J. Luo, and T. R. Shrout, J. Appl. Phys. 108, 034106 (2010).

${ }^{226}$ Y. P. Guo, H. S. Luo, D. Ling, H. Q. Xu, T. H. He, and Z. W. Yin, J. Phys.: Condens. Matter. 15, L77 (2003).

${ }^{227}$ IEEE Standard on Piezoelectricity, "IEEE Standard on Piezoelectricity," ANSI/IEEE Std 176 (1987).

${ }^{228}$ S. Liu, W. Ren, B. Mukherjee, S. J. Zhang, T. R. Shrout, P. W. Rehrig and W. Hackenberger, Appl. Phys. Lett., 832886 (2003).

${ }^{229}$ K. Yao and F. E. H. Tay, IEEE Trans. Ultrason. Ferroelectr. Freq. Control 50, 113 (2003).

${ }^{230}$ S. Emeterio, Ferroelectrics 293, 237 (2003).

${ }^{231}$ R. Holland and E. P. EerNisse, IEEE Trans. Sonics Ultrason. SU-16, 173 (1969).

${ }^{232}$ K. W. Kwok, H. Chan, and C. Choy, IEEE Trans. Ultrason. Ferroelectr. Freq. Control 44, 733 (1997).

${ }^{233}$ J. G. Smits, IEEE Trans. Sonics Ultrason. SU-23, 393 (1976).

${ }^{234}$ F. Li, Z. Xu, X. Wei, L. Jin, C. H. Zhang, J. J. Gao, and X. Yao, J. Phys. D: Appl. Phys. 42, 072001 (2009).

${ }^{235}$ S. J. Zhang, F. Li, J. Luo, R. Xia, W. Hackenberger, and T. R. Shrout, Appl. Phys. Lett. 97, 132903 (2010).

${ }^{236}$ H. Cao, V. H. Schmidt, R. Zhang, W. Cao, and H. Luo, J. Appl. Phys. 96, 549 (2004).

${ }^{237} \mathrm{~K}$. Uchino and S. Hirose, IEEE Trans. Ultrason. Ferroelectr. Freq. Control 48, 307 (2001).

${ }^{238}$ X. H. Du, Q. M. Wang, and K. Uchino, IEEE Trans. Ultrason. Ferroelectr. Freq. Control 50, 312 (2003).

${ }^{239}$ R. Sabat, B. K. Mukherjee, W. Ren, and G. M. Yang, J. Appl. Phys. 101, 064111 (2007).

${ }^{240}$ X. C. Geng, T. Ritter, and S. Park, in IEEE Ultrasonic Symposium (IEEE, Piscataway, NJ, 1998), pp. 571-574.

${ }^{241}$ S. Zhang, S. Lee, D. Kim, H. Lee, and T. R. Shrout, J. Am. Ceram. Soc. 91, 683 (2008).

${ }^{242}$ W. Jiang, R. Zhang, B. Jiang, and W. Cao, Ultrasonics 41, 55 (2003).

${ }^{243}$ S. Zhang, G. Liu, W. Jiang, J. Luo, W. Cao, and T. Shrout, J. Appl. Phys. 110, 064108 (2011).

${ }^{244}$ G. Liu, W. H. Jiang, J. Q. Zhu, and W. W. Cao, Appl. Phys. Lett. 99, 162901 (2011).

${ }^{245}$ R. Zhang, B. Jiang, W. Jiang, and W. Cao, Appl. Phys. Lett. 89, 242908 (2006).

${ }^{246}$ D. Damjanovic, M. Budimir, M. Davis, and N. Setter, Appl. Phys. Lett. 83, 527 (2003)

${ }^{247}$ M. Davis, M. Budimir, D. Damjanovic, and N. Setter, J. Appl. Phys. 101, 054112 (2007).

${ }^{248}$ R. Zhang, B. Jiang, and W. Cao, Appl. Phys. Lett. 82, 3737 (2003).

${ }^{249}$ M. Davis, D. Damjanovic, and N. Setter, J. Appl. Phys. 100, 084103 (2006).

${ }^{250}$ S. Zhang, N. Sherlock, R. Meyer, Jr., and T. Shrout, Appl. Phys. Lett. 94, 162906 (2009).

${ }^{251}$ P. Han, W. Yan, J. Tian, X. Huang, and H. Pan, Appl. Phys. Lett. 86, 052902 (2005).

${ }^{252}$ D. J. V. Tol and R. J. Meyer, Jr., "Acoustic transducer," US patent 7615912 (10 November 2009).

${ }^{253}$ S. J. Zhang, F. Li, W. H. Jiang, J. Luo, R. J. Meyer, Jr., W. W. Cao, and T. R. Shrout, Appl. Phys. Lett. 98, 182903 (2011).

${ }^{254}$ S. J. Zhang, W. H. Jiang, R. J. Meyer, Jr., F. Li, J. Luo, and W. W. Cao, J. Appl. Phys. 110, 064106 (2011).

${ }^{255}$ S. Goljahi, J. Gallagher, S. J. Zhang, J. Luo, R. Sahul, W. Hackenberger, and C. S. Lynch, "A relaxor ferroelectric single crystal cut resulting in 
large $d_{36}$ and zero $d_{31}$ for shear mode accelerometer applications," Smart Materials and Structures (submitted).

${ }^{256} \mathrm{~K}$. K. Deng, "Underwater acoustic vector sensor using transverse response free, shear mode, PMN-PT crystal," US patent US7066026 (27 June 2006).

${ }^{257}$ Z. Y. Feng, X. Y. Zhao, and H. S. Luo, J. Appl. Phys. 100, 024104 (2006).

${ }^{258}$ X. Y. Zhao, B. J. Fang, H. Cao, Y. P. Guo, and H. S. Luo, Mater. Sci. Eng. B 96, 254 (2002).

${ }^{259}$ Y. P. Guo, H. S. Luo, K. Chen, H. Q. Xu, X. W. Zhang, and Z. W. Yin, J. Appl. Phys. 92, 6134 (2002).

${ }^{260}$ J. Tian and P. D. Han, IEEE Trans. Ultrason. Ferroelectr. Freq. Control 54, 1895 (2007).

${ }^{261}$ M. Budimir, D. Damjanovic, and N. Setter, J. Appl. Phys. 94, 6753 (2003).

${ }^{262}$ M. J. Haun, E. Furman, S. J. Jang, and L. E. Cross, Ferroelectrics 99, 13 (1989).

${ }^{263}$ M. Davis, D. Damjanovic, and N. Setter, J. Appl. Phys. 96, 2811 (2004).

${ }^{264}$ R. W. Whatmore, Rep. Prog. Phys. 49, 1335 (1986).

${ }^{265}$ Y. X. Tang, X. M. Wan, X. Y. Zhao, X. M. Pan, D. Lin, H. S. Luo, J. L. Sun, X. J. Meng, and J. H. Zhu, J. Appl. Phys. 98, 084104 (2005).

${ }^{266}$ Y. X. Tang, X. Y. Zhao, X. Q. Feng, W. Q. Jin, and H. S. Luo, Appl. Phys. Lett. 86, 082901 (2005).

${ }^{267}$ L. H. Liu, X. Wu, X. Y. Zhao, X. Q. Feng, W. P. Jing, and H. S. Luo, IEEE Trans. Ultrason. Ferroelectr. Freq. Control 57, 2154 (2010).

${ }^{268}$ L. Liu, X. Li, X. Wu, Y. Wang, W. Di, D. Lin, X. Zhao, H. Luo, and N. Neumann, Appl. Phys. Lett. 95, 192903 (2009).

${ }^{269}$ X. Y. Zhao, X. Wu, L. H. Liu, H. S. Luo, N. Neumann, and P. Yu, Phys. Status Solidi A 208, 1061 (2011).

${ }^{270}$ Y. X. Tang, L. H. Luo, Y. M. Jia, H. S. Luo, X. Y. Zhao, H. Q. Xu, D. Lin, J. L. Sun, X. J. Meng, J. H. Zhu, and M. Es-Souni, Appl. Phys. Lett. 89, 162906 (2006).

${ }^{271}$ Y. Lu, Z. Cheng, S. E. Park, S. F. Liu, and Q. M. Zhang, Jpn. J. Appl. Phys., Part 1. 39, 141 (2000).

${ }^{272}$ X. Wan, D. Y. Wang, X. Y. Zhao, H. S. Luo, H. L. W. Chan, and C. L. Choy, Solid State Conmmun. 134, 547 (2005).

${ }^{273}$ E. W. Sun, Z. Wang, R. Zhang, and W. W. Cao, Opt. Mater. 33, 549 (2011)

${ }^{274}$ X. Wan, H. Xu, T. He, D. Lin, and H. S. Luo, J. Appl. Phys. 93, 4766 (2003).

${ }^{275}$ B. Noheda, D. E. Cox, G. Shirane, J. A. Gonzalo, L. E. Cross, and S.-E. Park, Appl. Phys. Lett. 74, 2059 (1999).

${ }^{276}$ L. Bellaiche, A. Garcia, and D. Vanderbilt, Phys. Rev. B 64, 060103 (2001)

${ }^{277}$ D. Damjanovic, IEEE Trans. Ultrason. Ferroelectr. Freq. Control 56, 1574 (2009).

${ }^{278}$ G. Y. Xu, J. S. Wen, C. Stack, and P. M. Gehring, Nature Mater. 7, 562 (2008)

${ }^{279}$ R. Guo, L. Cross, S. Park, B. Noheda, D. Cox, and G. Shirane, Phys. Rev. Lett. 84, 5423 (2000).

${ }^{280}$ G. S. Xu, H. S. Luo, H. Q. Xu, and Z. W. Yin, Phys. Rev. B. 64, 020102(R) (2002).

${ }^{281}$ B. Noheda, D. E. Cox, G. Shirane, S.-E. Park, L. E. Cross, and Z. Zhong, Phys. Rev. Lett. 86, 3891 (2001).

${ }^{282}$ B. Noheda, D. E. Cox, G. Shirane, J. Gao, and Z.-G. Ye, Phys. Rev. B. 66, 054104 (2002)

${ }^{283}$ D. La-Orauttapong, B. Noheda, Z.-G. Ye, P. M. Gehring, J. Toulouse, D. E. Cox, and G. Shirane, Phys. Rev. B. 65, 144101 (2002).

${ }^{284}$ D. Vanderbilt and M. H. Cohen, Phys. Rev. B. 63, 094108 (2001).

${ }^{285}$ Y. Jin, Y. Wang, A. Khachaturyan, J. Li, and D. Viehland, Phys. Rev. Lett. 91, 197601 (2003).

${ }^{286}$ H. F. Wang, B. Jiang, T. Shrout, and W. Cao, IEEE Trans. Ultrason. Ferroelectr. Freq. Control 51, 908 (2004).

${ }^{287}$ B. Noheda, Z. Zhong, D. E. Cox, G. Shirane, S. E. Park, and P. Rehrig, Phys. Rev. B 65, 224101 (2002).

${ }^{288}$ D. Viehland, J. Appl. Phys. 88, 4794 (2000).

${ }^{289}$ E. H. Kisi, R. O. Piltz, J. S. Forrester, and C. J. Howard, J. Phys.: Condens. Matter 15, 3631 (2003).

${ }^{290}$ C. Boulesteix, F. Varnier, A. Llebaria, and E. Husson, J. Solid State Chem. 108, 141 (1994).

${ }^{291}$ J. S. Wen, G. Y. Xu, C. Stock, and P. M. Gehring, Appl. Phys. Lett. 93, 082901 (2008)

${ }^{292}$ D. Damjanovic, Appl. Phys. Lett. 97, 062906 (2010).
${ }^{293}$ K. Carl and K. H. Hardtl, Ferroelectrics 17, 473 (1978).

${ }^{294}$ S. Priya and K. Uchino, Jpn. J. Appl. Phys. 42, 531 (2003).

${ }^{295}$ N. P. Sherlock, "Relaxor-PT single crystals for broad bandwidth, high power sonar projectors," Ph.D. dissertation (The Pennsylvania State University, PA, 2010).

${ }^{296}$ Y. Zhuang, S. O. Ural, R. Gosain, S. Tuncdemir, A. Amin, and K. Uchino, Appl. Phys. Express 2, 121402 (2009).

${ }^{297}$ F. Li, S. J. Zhang, Z. Xu, X. Wei, J. Luo, and T. R. Shrout, Appl. Phys. Lett. 96, 192903 (2010).

${ }^{298}$ W. Gao, D. F. Jin, W. C. Wei, H. J. Maris, J. Tian, X. L. Huang, and P. D. Han, J. Appl. Phys. 102, 084104 (2007).

${ }^{299}$ Z. R. Li, Z. Xu, X. Yao, and Z.-Y. Cheng, J. Appl. Phys. 104, 024112 (2008).

${ }^{300}$ F. Wang, S. Or, X. Zhao, and H. Luo, J. Phys. D: Appl. Phys. 42, 182001 (2009).

${ }^{301}$ A. Amin, IEEE Trans. Ultrason. Ferroelectr. Freq. Control. 52, 1632 (2005).

${ }^{302}$ A. Amin, E. McLaughlin, H. Robinson, and L. Ewart, IEEE Trans. Ultrason. Ferroelectr. Freq. Control. 54, 1090 (2007).

${ }^{303}$ A. Amin and L. E. Cross, J. Appl. Phys. 98, 094113 (2005).

${ }^{304}$ C. Okawara and A. Amin, Appl. Phys. Lett. 95, 072902 (2009).

${ }^{305}$ M. Shanthi and L. C. Lim, Appl. Phys. Lett. 95, 102901 (2009).

${ }^{306}$ M. Shanthi, D.-H. Lin, R. See, W. S. Chang, and L. C. Lim, Sens. Actuators, A 168, 112 (2011).

${ }^{307}$ M. Shanthi and L. C. Lim, J. Appl. Phys. 106, 114116 (2009).

${ }^{308}$ A. A. Bokov and Z.-G. Ye, Appl. Phys. Lett. 92, 082901 (2008).

${ }^{309}$ E. A. Mclaughlin, T. Q. Liu, and C. S. Lynch, Acta. Mater. 52, 3849 (2004).

${ }^{310}$ E. A. Mclaughlin, T. Q. Liu, and C. S. Lynch, Acta. Mater. 53, 4001 (2005).

${ }^{311}$ D. Damjanovic and M. Demartin, J. Phys.: Condens. Matter. 9, 4943 (1997).

${ }^{312}$ F. Li, Z. Xu, X. Wei, X. Yao, and L. Jin, Appl. Phys. Lett. 93, 192904 (2008).

${ }^{313}$ F. Li, S. J. Zhang, Z. Xu, Z. R. Li, and X. Y. Wei, J. Adv. Dielectr. 1, 303 (2011).

${ }^{314}$ D. Viehland, L. Ewart, J. Powers, and J. F. Li, J. Appl. Phys. 90, 2479 (2001).

${ }^{315}$ D. Viehland and J. F. Li, J. Appl. Phys. 94, 7719 (2003).

${ }^{316}$ D. Viehland, J. Am. Ceram. Soc. 89, 775 (2006).

${ }^{317}$ D. Viehland and J. Powers, Appl. Phys. Lett. 78, 3112 (2001).

${ }^{318}$ Q. Wan, C. Chen, and Y. P. Shen, J. Mater. Sci. 41, 2993 (2006).

${ }^{319}$ Q. Wan, C. Chen, and Y. P. Shen, J. Appl. Phys. 98, 024103 (2005).

${ }^{320}$ S. J. Zhang, J. Luo, R. Shanta, D. W. Snyder, and T. R. Shrout, in 15th IEEE International Symposium Appl. Ferroeletr. (2006), pp. 261-264.

${ }^{321}$ N. Yasuda, Y. Itoh, H. Ohwa, M. Matushita, Y. Yamashita, M. Iwata, and Y. Ishibashi, Jpn. J. Appl. Phys. 43, 6675 (2004).

${ }^{322}$ N. Yasuda, M. D. M. Rahman, H. Ohwa, M. Matsushita, Y. Yamashita, M. Iwata, H. Terauchi, and Y. Ishibashi, Appl. Phys. Lett. 89, 192903 (2006).

${ }^{323}$ N. Yasuda, S. Suzuki, M. M. Rahman, H. Ohwa, M. Matsushita, Y. Yamashita, M. Iwata, H. Terauchi, and Y. Ishibashi, J. Appl. Phys. 103, 064509 (2008).

${ }^{324}$ A. Bernal, S. J. Zhang, and N. Bassiri-Gharb, Appl. Phys. Lett. 95, 142911 (2009).

${ }^{325}$ M. Davis, D. Damjanovic, and N. Setter, J. Appl. Phys. 95, 5679 (2004).

${ }^{326}$ S. J. Zhang, F. Li, J. Luo, R. Xia, W. Hackenberger, and T. R. Shrout, IEEE Trans. Ultrason. Ferroelectr. Freq. Control 58, 274 (2011).

${ }^{327}$ X. J. Lou, Appl. Phys. Lett. 94, 072901 (2009).

${ }^{328}$ M. Ozgul, S. Trolier-McKinstry, and C. A. Randall, J. Appl. Phys. 95, 4296 (2004).

${ }^{329}$ M. Ozgul, K. Takemura, S. Trolier-McKinstry, and C. A. Randall, J. Appl. Phys. 89, 5100 (2001).

${ }^{330}$ J. K. Lee, J. Y. Yi, and K. Hong, J. Appl. Phys. 96, 7471 (2004).

${ }^{331}$ Y. Zhang, Z. Xu, T. Hung, H. Luo, and Q. Yin, J. Eur. Ceram. Soc. 24, 2983 (2004).

${ }^{332}$ S. Zhang, J. Luo, F. Li, R. Meyer, W. Hackenberger, and T. Shrout, Acta Mater. 58, 3773 (2010).

${ }^{333}$ K. Takemura, M. Ozgul, V. Bornand, S. Trolier-McKinstry, and C. Randall, J. Appl. Phys. 88, 7272 (2000).

${ }^{334}$ Z. Xu, X. Tan, P. Han, J. K. Shang, Appl. Phys. Lett. 76, 3732 (2000).

${ }^{335}$ X. Tan, Z. Xu, J. K. Shang, and P. Han, Appl. Phys. Lett. 77, 1529 (2000). 
${ }^{336}$ F. Fang, W. Yang, F. C. Zhang, and H. Qing, Appl. Phys. Lett. 91, 081903 (2007).

${ }^{337}$ F. Fang, W. Yang, and X. Luo, J Appl. Phys. 106, 094107 (2009).

${ }^{338} \mathrm{~J}$. Chen and R. Panda, in IEEE Ultrasonic Symposium (IEEE, Piscataway, NJ, 2005), pp. 235-240.

${ }^{339}$ S. M. Rhim, M. C. Shin, and S. G. Lee, "Piezoelectric single crystals for medical ultrasonic transducers," in Handbook of Advanced Dielectric, Piezoelectric and Ferroelectric Materials - Synthesis, Characterization and Applications, edited by Z. G. Ye (Woodhead, Cambridge, England, 2008), pp. 101-129.

${ }^{340}$ X. C. Geng, T. Ritter, and K. K. Shung, Proc. SPIE 3664, 24 (1999).

${ }^{341}$ T. Ritter, X. C. Geng, K. K. Shung, P. D. Lopath, S. E. Park, and T. R. Shrout, IEEE Trans. Ultrason. Ferroelectr. Freq. Control 47, 792 (2000)

${ }^{342}$ K. A. Snook, X. N. Jiang, W. S. Hackenberger, X. C. Geng, A. Winder, and F. Forsberg, in IEEE Ultrasonic Symposium (IEEE, Piscataway, NJ, 2007), pp. 292-295.

${ }^{343}$ T. Ritter, K. K. Shung, X. Geng, H. Wang, and T. R. Shrout, in IEEE Ultrasonic Symposium (IEEE, Piscataway, NJ, 1998), pp. 1851-1854.

${ }^{344}$ S. Saitoh, T. Kobayashi, K. Harada, S. Shimanuki, and Y. Yamashita, Jpn. J. Appl. Phys. 37, 3053 (1998).

${ }^{345}$ Y. Hosono and Y. Yamashita, J. Electroceram. 17, 577 (2006).

${ }^{346}$ Q. F. Zhou, X. Xu, E. J. Gottlieb, L. Sun, J. M. Cannata, H. Ameri, M. S. Humayun, P. Han, and K. K. Shung, IEEE Trans. Ultrason. Ferroelectr. Freq. Control 54, 668 (2007)

${ }^{347}$ K. C. Cheng, H. L. W. Chan, C. L. Choy, Q. R. Yin, H. S. Luo, and Z. W. Yin, IEEE Trans. Ultrason. Ferroelectr. Freq. Control 50, 1177 (2003)

${ }^{348}$ T. R. Gururaja, R. K. Panda, J. Chen, and H. Beck, in IEEE Ultrasonic Symposium (IEEE, Piscataway, NJ, 1999), pp. 969-972.

${ }^{349}$ F. J. Kumar, L. C. Lim, S. P. Lim and K. H. Lee, IEEE Trans. Ultrason. Ferroelectr. Freq. Control 50, 203 (2003).

${ }^{350} \mathrm{C}$. Okaley and M. Zipparo, in IEEE Ultrasonic Symposium (IEEE, Piscataway, NJ, 2000), pp. 1157-1160.

${ }^{351}$ S. Rhim, H. Jung, S. Kim and S. Lee, in IEEE Ultrasonic Symposium (IEEE, Piscataway, NJ, 2002), pp. 1143-1146.

${ }^{352}$ S. Rhim, H. Kim, H. Jung, S. Kim and S. Lee, in IEEE Ultrasonic Symposium (IEEE, Piscataway, NJ, 2003), pp. 782-785.

${ }^{353}$ Z. Xu, F. Chen, Z. Xi, Z. Li, L. Cao, Y. Feng, and X. Yao, Ceram. Int. 30, 1777 (2004).

${ }^{354}$ W. Hackenberger, X. Jiang, P. Rehrig, X. Geng, A. Winder, and F. Prosberg, in IEEE Ultrasonic Symposium (IEEE, Piscataway, NJ, 2003), pp. $778-781$.

${ }^{355}$ X. N. Jiang, K. Snook, C. Hu, X. Geng, R. Liu, J. Welter, K. Shung, and W. Hackenberger, Proc. SPIE 7294, 729403 (2009).

${ }^{356}$ X. N. Jiang, K. Snook, A. Cheng, W.S., Hackenberger, and X. Geng, in IEEE Ultrasonics Symposium (IEEE, Piscataway, NJ, 2008), pp. $164-167$

${ }^{357}$ J. Yuan, S. Rhee, and X.N. Jiang, in IEEE Ultrasonics Symposium (IEEE, Piscataway, NJ, 2008), pp. 682-685.

${ }^{358}$ P. W. Rehrig, X. N. Jiang, W. S. Hackenberger, J. R. Yuan, and R. Romley, "Micromachined imaging transducer," U.S. patent US7622853 (24 November 2009)

${ }^{359}$ X. N. Jiang, K. Snook, and W.S. Hackenberger, Proc. SPIE 6531, 65310F (2007)

${ }^{360}$ X. N. Jiang, K. Snook, T. Walker, A. Portune, R. Haber, X. Geng, J. Wetter, and W. S. Hackenberger, Proc. SPIE 6934, 69340D (2008).

${ }^{361}$ J. Yuan, X. N. Jiang, P. Cao, K. Snook, P. Rehrig, and W. Hackenberger, in IEEE Ultrasonics Symposium (IEEE, Piscataway, NJ, 2006), pp. 264-268

${ }^{362}$ D. Zhou, K. F. Cheung, Y. Chen, S. T. Lau, Q. F. Zhou, K. K. Shung, H. S. Luo, J. Y. Dai, and H. L. W. Chan, IEEE Trans. Ultrason. Ferroelectr. Freq. Control 58, 477 (2011)

${ }^{363}$ P. Sun, Q. F. Zhou, B. P. Zhu, D. W. Wu, C. L. Hu, J. M. Cannata, J. Tian, P. D. Han, G. F. Wang, and K. K. Shung, IEEE Trans. Ultrason. Ferroelectr. Freq. Control 56, 2760 (2009).

${ }^{364}$ Q. F. Zhou, D. W. Wu, J. Jin, C. H. Hu, X. C. Xu, J. Williams, J. M. Cannata, L. C. Lim, and K. K. Shung, IEEE Trans. Ultrason. Ferroeletr. Freq. Control 55, 1369 (2008).

${ }^{365}$ K. K. Shung, J. M. Cannata, and Q. F. Zhou, J. Electroceram. 19, 139 (2007)

${ }^{366}$ J. Chen, R. K. Panda, T. R. Gururaja, and H. Beck, "Single crystal thickness and width cuts for enhanced ultrasonic transducer," U.S. patent US6465937 (15 October 2002).
${ }^{367}$ J. Chen, R. Panda, P. G. Rafter, and T. R. Gururaja, "Wideband piezoelectric transducer for harmonic imaging," U.S. patent US6532819 (18 March 2003)

${ }^{368}$ X. N. Jiang, J. R. Yuan, A. Cheng, K. Snook, P. Cao, P. W. Rehrig, W. S. Hackenberger, G. Lavalelle, X. Geng, and T. Shrout, in IEEE Ultrasonic Symposium (IEEE, Piscataway, NJ, 2006), pp. 918-921.

${ }^{369}$ J. Tian and P. D. Han, "PMN-PT based piezoelectric single crystals and composites," in Proceedings of the 2011 IEEE Intern. Symp. Appl. Ferroeletr., Vancouver, Canada, 24-27 July 2011.

${ }^{370}$ J. F. Tressler, T. R. Howarth, and D. Huang, J. Acoust. Soc. Am. 119, 879 (2006).

${ }^{371}$ J. M. Powers, M. B. Moffett, and F. Nussbaum, in 12th IEEE Intern. Symp. Appl. Ferroelectr. (IEEE, Piscataway, NJ, 2000), pp. 351-354.

${ }^{372}$ K. A. Snook, P. W. Rehrig, W. S. Hackenberger, X. N. Jiang, R. J. Meyer, and D. Markley, Proc. SPIE 5761, 263 (2005).

${ }^{373}$ R. J. Meyer, Jr., T. Montgomery, and W. Hughes, in MTS/IEEE Oceans' 02 (IEEE, Piscataway, NJ, 2002), pp. 2328-2331.

${ }^{374}$ K. A. Snook, P. W. Rehrig, W. S. Hackenberger, X. N. Jiang, R. J. Meyer, Jr., and D. Markley, Proc. SPIE 6170, 61700H (2006).

${ }^{375}$ K. A. Snook, P. W. Rehrig, W. S. Hackenberger, R. J. Meyer, Jr., and D. Markley, in IEEE Ultrasonic Symposium (IEEE, Piscataway, NJ, 2006), pp. 359-361.

${ }^{376}$ R. J. Meyer, Jr., "Progress in single crystal sonar projector integration," in 2011 IEEE Intern. Symp. Appl. Ferroelectr., Vancouver, Canada, 2427 July 2011.

${ }^{377}$ H. C. Robinson, "Single crystal projectors for compact, broadband sonar," in Proceedings of the 2011 IEEE Intern. Symp. Appl. Ferroelectr., Vancouver, Canada, 24-27 July 2011.

${ }^{378}$ H. C. Robinson, J. R. Torres, R. S. Janus, K. C. Benjamin, and J. A. Szelag, "Single crystal flexural transducers for broadband LF sonar," in Proceedings of the US Navy Workshop on Acoustic Transduction Materials and Devices, State College, PA, 12-14 May 2009.

${ }^{379}$ M. B. Moffett, H. C. Robinson, J. M. Powers, and P. D. Baird, J. Acoust. Soc. Am. 121, 2591 (2007)

${ }^{380}$ K. Snook, B. Dunkin, W. Hackenberger, R. Meyer, Jr., and D. Markley, "Production of single crystal transducers for sonar platforms," in Proceedings of the US Navy Workshop on Acoustic Transduction Materials and Devices, State College, PA, 12-14 May 2009.

${ }^{381}$ R. J. Meyer, Jr., D. C. Markley, and N. P. Sherlock, "High power single crystal based projectors," in Proceedings of the US Navy Workshop on Acoustic Transduction Materials and Devices, State College, PA, 12-14 May 2009.

${ }^{382}$ R. J. Meyer, Jr., T. M. Tremper, D. C. Markley, D. Van Tol, P. Han, and J. Tian, "Low profile, broad-bandwidth projector design using d36 shear mode," in Proceedings of the US Navy Workshop on Acoustic Transduction Materials and Devices, State College, PA, 11-13 May 2010.

${ }^{383}$ L. C. Lim, J. Jin and K. K. Rajan, "PZN-PT single crystal underwater devices," in proceedings of the US Navy Workshop on Acoustic Transduction Materials and Devices, State College, PA, 12-14 May 2009.

${ }^{384}$ L. C. Lim and J. Jing, "New generation single crystal underwater transducers," in proceedings of the International Workshop on Acoustic Transduction Materials and Devices, State College, PA, 10-12 May 2011.

${ }^{385} \mathrm{C}$. H. Sherman and J. L. Butler, Transducers and Arrays for Underwater Sound (Springer, NY, 2007).

${ }^{386}$ D. P. Skinner, R. E. Newnham, and L. E. Cross, Mater. Res. Bull. 13, 599 (1978).

${ }^{387}$ R. E. Newnham, D. P. Skinner, and L. E. Cross, Mater. Res. Bull. 13, 525 (1978).

${ }^{388}$ A. Safari, J. Phys. III 4, 1129 (1994).

${ }^{389}$ V. F. Janas and A. Safari, J. Am. Ceram. Soc. 78, 2945 (1995).

${ }^{390}$ H. J. Lee, S. J. Zhang, and T. R. Shrout, J. Appl. Phys. 107, 124107 (2010).

${ }^{391}$ H. J. Lee, S. J. Zhang, J. Luo, F. Li, and T. R. Shrout, Adv. Funct. Mater. 20, 3154 (2010).

${ }^{392}$ D. Lin, H. Lee, S. Zhang, F. Li, Z. Li, Z. Xu, and T. Shrout, Scr. Mater. 64, 1149 (2011).

${ }^{393}$ G. Gautschi, Piezoelectric Sensorics (Springer, Berlin, 2002).

${ }^{394}$ J. F. Fernandez, A. Dogan, J. T. Fielding, K. Uchino, and R. E. Newnham, Sens. Actuators, A 65, 228 (1998).

${ }^{395}$ Z. Li, A. Huang, G. Luan, and J. Zhang, Ultrasonics 44, e759 (2006).

${ }^{396}$ W. A. Smith, IEEE Trans. Ultrason. Ferroelectr. Freq. Control 40, 41 (1993).

${ }^{397}$ V. Y. Topolov and A. V. Krivoruchko, J. App. Phys. 105, 074105 (2009).

${ }^{398}$ V. Y. Topolov, A. V. Krivoruchko, C. R. Bowen, and A. A. Panich, Ferroelectrics 400, 410 (2010). 
${ }^{399}$ A. V. Krivoruchko and V. Y. Topolov, J. Phys. D: Appl. Phys. 40, 7113 (2007).

${ }^{400} \mathrm{P}$. Wlodkowski, K. Deng, M. Kahn, and M. Chase, in 12th IEEE Intern. Symp. Appl. Ferroelectr. (IEEE, Piscataway, NJ, 2001), pp. 565-567.

${ }^{401}$ P. Wlodkowski, K. Deng, and M. Kahn, Sens. Actuators, A 90, 125 (2001).

${ }^{402}$ L. Zou and K. Deng, "High sensitivity, low noise piezoelectric flexural sensing structure using $<011>$ poled relaxor-based piezoelectric single crystals," U.S. patent US7104140 (12 September 2006).

${ }^{403}$ S. Tadigadaps and K. Mateti, Meas. Sci. Technol. 20, 092001 (2009).

${ }^{404}$ L. C. Lim, J. Jing, Y. X. Xia, and Z. M. Lee, in Proceedings of the Naval Technology Seminar, (NTS 2011), Singapore, 20 May 2011.

${ }^{405}$ S. C. Woody, S. Smith, X. Jiang, and P. W. Rehrig, Rev. Sci. Instrum. 76, 075112 (2005)

${ }^{406}$ X. N. Jiang, W. Cook, and W. S. Hackenberger, Proc. SPIE 7439, $74390 Z$ (2009)

${ }^{407}$ X. N. Jiang, P. W. Rehrig, J. Luo, W. Hackenberger, S. J. Zhang, and T. R. Shrout, Proc. SPIE 6170, 61700G (2006).

${ }^{408}$ X. N. Jiang, P. W. Rehrig, W. S. Hackenberger, S. Perini, M. Lanagan, X. X. Xi, E. Furman, E. Prophet, B. Willemsen, and B. Hammond, Adv. Cryog. Eng. 51A, 928 (2005).

${ }^{409}$ X. N. Jiang, P. W. Rehrig, W. S. Hackenberger, E. Smith, S. Dong, D. Viehland, J. Moore, and B. Patrick, Proc. SPIE 5761, 253 (2005).

${ }^{410}$ X. N. Jiang, P. W. Rehrig, W. S. Hackenberger, J. Moore, S. Chodimella, and B. Patrick, Proc. ASME 3, IMECE-60504 (2004).

${ }^{411}$ Z. Y. Feng, T. H. He, H. Q. Xu, H. S. Luo, and Z. W. Yin, Solid State Commun. 130, 557 (2004).

${ }^{412}$ R. J. Meyer, Jr., A. Dogan, C. Yoon, S. M. Pilgrim, and R. E. Newnham, Sens. Actuators, A 87, 157 (2001).

${ }^{413}$ X. N. Jiang, P. W. Rehrig, W. Hackenberger, and T. Shrout, Proc. SPIE 5053, 436 (2003).

${ }^{414}$ X. N. Jiang, S. Dong, P. W. Rehrig, W. S. Hackenberger, and D. Viehland, in IEEE Ultrasonic Symposium (IEEE, Piscataway, NJ, 2004), pp. 1314-1317.
${ }^{415}$ X. N. Jiang, P. W. Rehrig, W. S. Hackenberger, and T. R. Shrout, Adv. Cryog. Eng. 51B, 1783 (2005).

${ }^{416}$ S. X. Dong, L. Yan, D. Viehland, X. N. Jiang, and W. S. Hackenberger, Appl. Phys. Lett. 92, 153504 (2008).

${ }^{417}$ S. X. Dong, L. Yan, N. G. Wang, D. Viehland, X. N. Jiang, P. W. Rehrig, and W. S. Hackenberger, Appl. Phys. Lett. 86, 053501 (2005).

${ }^{418}$ L. Luo, H. Zhu, C. Zhao, H. Wang, and H. Luo, Appl. Phys. Lett. 90, 052904 (2007).

${ }^{419}$ M. Guo, S. X. Dong, B. Ren, and H. S. Luo, IEEE Trans. Ultrason. Ferroelectr. Freq. Control 57, 2596 (2010).

${ }^{420}$ K. Uchino, Acta Mater. 46, 3745 (1998).

${ }^{421}$ L. Lebrun, G. Sebald, B. Guiffard, C. Richard, D. Guyomar, and E. Pleska, Ultrasonics 42, 501 (2004).

${ }^{422}$ G. Sebald, L. Lebrun, B. Guiffard, and D. Guyomar, J. Eur. Ceram. Soc. 25, 2509 (2005).

${ }^{423}$ F. F. Wang, W. Z. Shi, Y. X. Tang, X. M. Chen, T. Wang, and H. S. Luo, Appl. Phys. A 100, 1231 (2010).

${ }^{424}$ M. A. Karami, O. Bilgen, D. J. Inman, and M. I. Friswell, IEEE Trans. Ultrason. Ferroelectr. Freq. Control 58, 1508 (2011).

${ }^{425}$ S. Priya and D. J. Inman, Energy Harvesting Technologies (Springer, NY, 2009).

${ }^{426}$ A. Erturk and D. J. Inman, Piezoelectric Energy Harvesting (Wiley, NY, 2011).

${ }^{427}$ K. Ren, Y. Liu, X. Geng, H. Hofmann, and Q. Zhang, IEEE Trans. Ultrason. Ferroelectr. Freq. Control 53, 631 (2006).

${ }^{428}$ G. Sebald, E. Lefeuvre, and D. Guyomar, IEEE Trans. Ultrason. Ferroelectr. Freq. Control 55, 538 (2008).

${ }^{429}$ A. Khodayari, S. Pruvost, G. Sebald, D. Guyomar, and S. Mohammadi, IEEE Trans. Ultrason. Ferroelectr. Freq. Control 56, 693 (2009).

${ }^{430}$ Y. X. Tang and H. S. Luo, J. Phys. D 42, 075406 (2009).

${ }^{431}$ Y. X. Tang and H. S. Luo, Infrared Phys. Technol. 52, 180 (2009).

${ }^{432}$ S. J. Zhang and T. R. Shrout, IEEE Trans. Ultrason. Ferroelectr. Freq. Control 57, 2138 (2010). 